\title{
Multi-omic network-based interrogation of rat liver metabolism following gastric bypass surgery featuring SWATH proteomics
}

\author{
Gautham Vivek Sridharan ${ }^{1}$, Matthew D’Alessandro ${ }^{1}$, Shyam Sundhar Bale ${ }^{1}$, Vicky Bhagat ${ }^{2}$, Hugo Gagnon ${ }^{3}$, \\ John M. Asara ${ }^{4}$, Korkut Uygun ${ }^{1}$, Martin L. Yarmush ${ }^{1} \&$ Nima Saeidi $^{1}$
}

\begin{abstract}
Morbidly obese patients often elect for Roux-en-Y gastric bypass (RYGB), a form of bariatric surgery that triggers a remarkable $30 \%$ reduction in excess body weight and reversal of insulin resistance for those who are type II diabetic. A more complete understanding of the underlying molecular mechanisms that drive the complex metabolic reprogramming post-RYGB could lead to innovative non-invasive therapeutics that mimic the beneficial effects of the surgery, namely weight loss, achievement of glycemic control, or reversal of non-alcoholic steatohepatitis (NASH). To facilitate these discoveries, we hereby demonstrate the first multi-omic interrogation of a rodent RYGB model to reveal tissue-specific pathway modules implicated in the control of body weight regulation and energy homeostasis. In this study, we focus on and evaluate liver metabolism three months following RYGB in rats using both SWATH proteomics, a burgeoning label free approach using high resolution mass spectrometry to quantify protein levels in biological samples, as well as MRM metabolomics. The SWATH analysis enabled the quantification of 1378 proteins in liver tissue extracts, of which we report the significant down-regulation of Thrsp and Acot13 in RYGB as putative targets of lipid metabolism for weight loss. Furthermore, we develop a computational graph-based metabolic network module detection algorithm for the discovery of non-canonical pathways, or sub-networks, enriched with significantly elevated or depleted metabolites and proteins in RYGB-treated rat livers. The analysis revealed a network connection between the depleted protein Baat and the depleted metabolite taurine, corroborating the clinical observation that taurine-conjugated bile acid levels are perturbed post-RYGB.
\end{abstract}

Keywords: Obesity; Roux-en-Y; Gastric Bypass; SWATH Proteomics; Metabolomics.

\section{INNOVATION}

The innovation of this work is two-fold: First is the application of SWATH proteomics, a state-of-the-art label free work flow using high resolution LC/MS-MS technology, to obtain physiological insight into the liver metabolic remodeling that occurs following gastric bypass, thereby elucidating the molecular mechanisms behind the weight loss and increased insulin sensitivity. Second is a novel computational approach to integrate metabolomics and proteomics data using a metabolic network-based algorithm that can efficiently capture and visualize differentially expressed metabolic enzyme levels along with the relative concentrations of metabolites, whose production and consumption are catalyzed by those enzymes. Hence, we present a generally applicable workflow of multi-omics analysis that can be applied to any in vivo or in vitro experimental system. We demonstrate the utilization of this innovation in the context of understanding the effects of rodent gastric bypass surgery, which can facilitate the discovery of novel therapeutic targets for metabolic diseases.

\section{INTRODUCTION}

Obesity, broadly characterized by excess accumulation of body fat, affects approximately $35 \%$ of the adult US population ${ }^{1}$ and is usually associated with several co-morbidities that define the metabolic syndrome, including type II diabetes, hypertension, and non-alcoholic fatty liver disease (NAFLD). Unfortunately, FDA-approved obesity therapeutics have historically been marred by safety concerns and newly approved drugs offer very modest efficacies. For example, Lorcaserin (Arena Pharmaceuticals/ Eisai), a serotonin receptor agonist modulating appetite, was recently approved for obesity in 2012, but clinical trials demonstrated only a 3-4\% placebo-adjusted sustained reduction in body weight ${ }^{2}$. In contrast, gastric bypass surgery remains a far more effective treatment option, where patients opting for the procedure to reconfigure gastrointestinal tract anatomy and enable food to bypass the stomach and parts of the intestine, sustain an average of $30 \%$ reduction in body weight post-operatively. Since Roux-en-Y gastric bypass (RYGB, the most common form of the surgery) stimulates the secretion of various hormones ${ }^{3}$ and results in

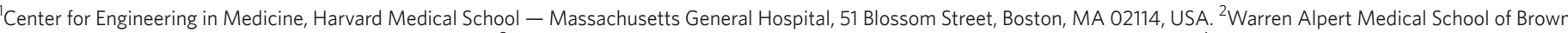

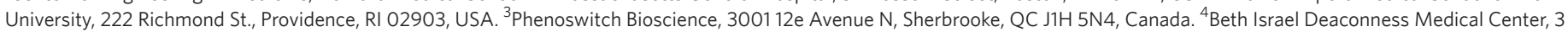
Blackfan Circle Rm 425, Boston, MA 02115, USA. Correspondence should be addressed to N.S. (nsaeidi@mgh.harvard.edu) or M.L.Y. (IReis@sbi.org).
} 
the reprogramming of metabolically active tissues, the rapid weight loss involves a more complex mechanism than can be solely explained by the reduction in food intake by patients post-surgery. Unfortunately, RYGB surgery, while therapeutically very effective, is highly invasive and presents risk of further complication. As such, understanding the molecular mechanisms of action that trigger the metabolic shifts following RYGB offers an avenue to discover novel non-invasive therapies against symptoms of the metabolic syndrome.

In this light, several clinical studies have been conducted on bariatric surgery patients to collect tissue, urine, and serum samples and experimentally quantify various biomolecules post-surgery using proteomics $^{4,5}$ and metabolomics ${ }^{6,7}$ approaches. However, access to intestinal, liver, and visceral fat tissue post-surgery is highly invasive and therefore information on the metabolic dynamics in these specific organs has largely been elusive based on human clinical studies. For further investigation of various metabolically active tissues following the surgery, rodent models for RYGB that mimic the weight loss and resolution of glycemic control in humans have offered a controlled experimental platform to capture the system-wide impacts of the surgery ${ }^{8-10}$. These animal models have offered insight into the effects of various gut-derived hormones such as Glp1, the impact of the gut-microbiota in modulating weight loss ${ }^{11}$, and the altered metabolism of bile acids, some of which have been implicated as agonists of FXR, a therapeutic target for NAFLD ${ }^{12}$. In particular, Saeidi and coworkers recently demonstrated the first mechanism of action for the rapid improvement in glycemic control following RYGB in rats, based on evidence that glucose uptake in intestinal tissue was highly up-regulated ${ }^{10}$.

The clinical observation that RYGB elicits a perturbation to bile acid metabolism and secretion suggests that a similar metabolic reprogramming of hepatocytes occurs following the surgery and further interrogation of the liver could help unravel the underlying mechanism of RYGB's long term effects. In this study, we exploit SWATH ${ }^{13}$ (Sequential Window Acquisition of all Theoretical fragments), a relatively new label-free mass spectrometry technique for targeted proteomics, for the global profiling of rat liver tissue metabolism three months following Sham and RYGB treatment. SWATH proteomics is increasingly being utilized for translational medicine applications ${ }^{14-16}$ since it offers an impressive coverage of the target proteome as recently demonstrated by Rosenberger and coworkers, who developed a SWATH-based assay capable of identifying 10,316 proteins from human samples ${ }^{17}$. Since a subset of the proteins identified using the technique are metabolic enzymes catalyzing the production and consumption of small molecule metabolites, we also complemented the SWATH data with targeted metabolomics analysis on the same liver tissue extracts using multiple reaction monitoring $(\mathrm{MRM})^{18}$. As with all omics analysis, interpretation of large multi-dimensional data to provide insightful biological insight remains challenging. We therefore also develop and present a novel computational network-based integration of proteomics and metabolomics data to identify reaction modules involved in metabolism that are enriched with statistically significant proteins and metabolites (between Sham and RYGB treated groups) using a local neighborhood search algorithm.

In summary, we report the quantification of 1378 proteins in liver extracts from rats subject to either Sham or RYGB treatment, of which 82 exhibited a statistically significant difference in expression between the experimental groups. A cursory analysis of the data revealed a perturbation to proteins involved in iron metabolism and a couple of proteins known to regulate lipid metabolism (Thrsp, Acot13), both of which have been previously validated by others as putative obesity targets based on body weight reduction in knockout mice. Moreover, a more rigorous analysis of the multi-omic integration using the network modularity approach revealed complex perturbations to modules otherwise classified as reactions from "one carbon folate metabolism" and "bile acid synthesis", the latter highlighting taurine conjugation of bile acids, which are known to correlate with obesity metrics in the clinic.
Our experimental and computational workflow should serve as a novel template for future studies in translational medicine featuring multi-omic integration to evaluate complex metabolic processes and discover novel therapeutic targets.

\section{RYGB MULTI-OMIC INTEGRATION: METHODOLOGY AND RESULTS}

\section{Physiological outcomes of RYGB and -omic workflow}

The anatomical reconstruction of RYGB in rats is highlighted in Fig. 1a-c and the surgical methods for both RYGB and sham surgery were similar to those previously described in $\operatorname{detail}^{9}$. Briefly, diet-induced obese (DIO) male Sprague Dawley rats (Charles River Laboratories) were fasted overnight and anesthetized using 2-4\% isoflurane. In the RYGB surgery, a small gastric pouch was created using a laparoscopic stapler (Ethicon, Cincinnati, $\mathrm{OH}$ ). The jejunum was transected and anastomosed to the gastric pouch to create the Roux Limb (RL) and the proximal transected end of the duodenum was anastomosed to the side of the distal jejunum to create the biliopancreatic limb (BP) restore intestinal tract continuity. The sham operation, which omits the step of the surgical procedure regarded as the therapeutic necessity, was performed on age and weight-matched rats. Briefly, this involved a laparotomy, where the ligament of Treitz is identified, and the same segment of the intestine was transected as in the real RYGB surgery. However, instead of constructing biliopancreatic and roux limbs, the two transected segments were simply anastamosed back together. A hole was constructed in the forestomach where the roux limb would be attached and then repaired, after which the laparotomy was closed ${ }^{10,19}$.

The RYGB-treated animals undergo a statistically significant $~ 25 \%$ weight loss (Fig. 1d) that was sustained for the entire duration of this study (3 months). In addition, histological samples demonstrate that while the liver in the sham animal contain large number of lipid droplets, which is a hallmark of hepatic steatosis, the condition is completely resolved in the RYGB animals (Fig. 1e,f). In this study, we present a generally applicable experimental and computational workflow to interrogate tissue metabolism of an in vivo system, which as highlighted in Fig. 1g, involves harvesting liver tissue biopsies and employing LC/MS-MS analytical platforms for targeted proteomics and metabolomics data.

\section{Coverage of SWATH proteomics}

The SWATH proteomics analysis is based on AB Sciex Tripletof LC/ MS-MS technology and is described in more detail in Supplementary Methods. The bioinformatics workflow used to map the high resolution MS/MS spectra to known trypsin-digested peptides enabled the relative quantification of 1378 proteins in rat liver tissue extracts in at least one of the Sham or RYGB treated animals. To determine the coverage and utility of SWATH proteomics for rat liver metabolism, we compiled a list of rat liver proteins belonging to each KEGG pathway ${ }^{20}$ that were detected by SWATH in at least one sample and computed the fractional coverage of each pathway by dividing the number of detected proteins by the total number of expressed genes in rat. For example, the pathway "Glyoxylate and dicarboxylate metabolism" yielded complete coverage, in that for all 28 rat genes catalogued in KEGG, the corresponding protein was quantified in rat liver using SWATH (Table 1). In contrast, out of the 1249 genes catalogued in "Olfactory transduction", only two proteins were detected in liver (Supplementary Table 5), which is unsurprising considering the liver does not function in smell detection.

\section{Effect of RYGB on rat liver proteome}

Of the 1378 proteins that were detected and quantifiable in rat liver extracts using SWATH, 45 proteins are statistically significantly elevated in RYGB samples compared to Sham and 37 are depleted in RYGB (Fig. 2a), for a total of 82 Statistically Significant Proteins (SSPs) based on the Wilcoxon rank test (the ranksum function in Matlab ${ }^{\circ}$ ). The Wilcoxon rank test is a 

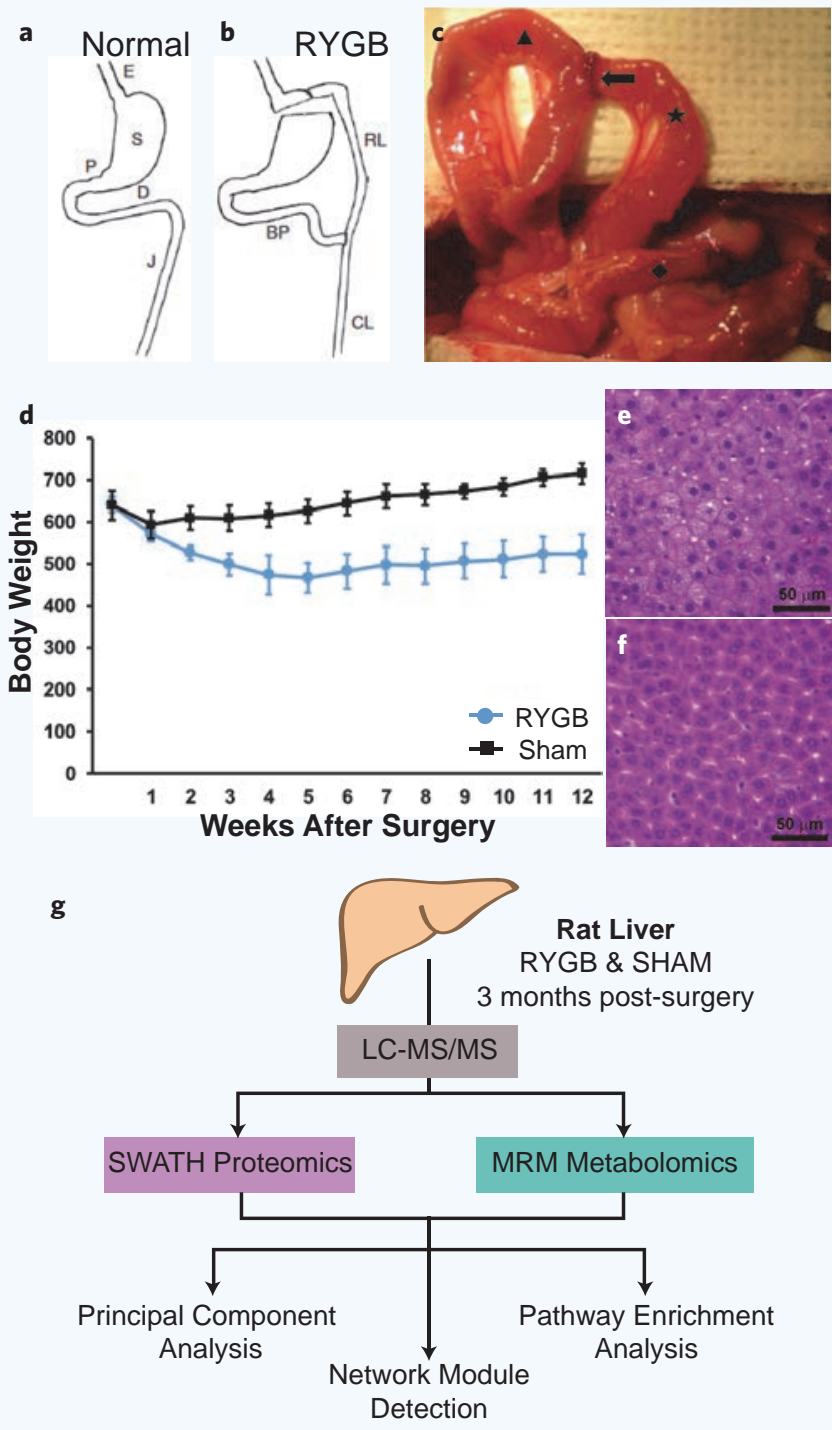

Figure 1 RYGB in rats induces systemic and hepatic-specific metabolic improvements. (a,b) Schematic drawing and (c) intraoperative image showing the anatomical reconfiguration of the gastrointestinal tract following RYGB with the following annotations: $\mathbf{\Delta}$ : common limb, -: site of anastamoses between the biliopancreatic limb and common limb, $\star$ : the biliopancreatic limb, $\bullet$ the roux limb (d) At three months post-surgery, the RYGB-treated rats sustainably have lost $\sim 25 \%$ of their pre-operative body weight. In contrast, the sham-treated rats continuously gained weight throughout the duration of the experiment. (e) Histological analysis of liver biopsies from both groups clearly demonstrate hepatocellular ballooning and steatosis in sham animals, which was completely resolved in RYGB-operated animals (f). (g) The multi-omic experimental workflow for the study.

non-parametric test to determine the likelihood that two samples are from the same distribution of equal medians, and is often used for in vivo experiments with small sample sizes where the residuals of the measurements are not normally distributed. The complete list of the calculated SSPs, along with a description of their function and relation to energy metabolism, or the metabolic syndrome is presented in Supplementary Table 1. Principal component analysis shows separation between the two treatment groups, with the separation driven by the second principal component (Fig. 2b). The absolute value of PC2's coefficients were sorted by magnitude and the
Table 1 Fractional coverage of KEGG pathways using SWATH proteomics in rat liver samples.

\begin{tabular}{lrcc}
\hline A KEGG pathway & $\begin{array}{c}{ }^{\mathrm{B}} \text { Rat } \\
\text { genes }\end{array}$ & $\begin{array}{c}{ }^{\mathrm{C}} \text { SWATH } \\
\text { proteins }\end{array}$ & ${ }^{\mathrm{D}}$ Coverage \\
\hline 1. Glyoxylate and dicarboxylate metabolism & 29 & 29 & 1.00 \\
2. Lysine biosynthesis & 2 & 2 & 1.00 \\
3. Citrate cycle (TCA cycle) & 32 & 29 & 0.91 \\
4. Propanoate metabolism & 32 & 28 & 0.88 \\
5. Valine, leucine, and isoleucine degradation & 56 & 42 & 0.75 \\
6. 2-oxocarboxylic acid metabolism & 19 & 14 & 0.74 \\
7. Fatty acid degradation & 47 & 33 & 0.70 \\
8. Primary bile acid biosynthesis & 16 & 11 & 0.69 \\
9. D-Glutamine and D-glutamate & 3 & 2 & 0.67 \\
$\quad$ metabolism & & & \\
10. Proteasome & 47 & 31 & 0.66 \\
\hline
\end{tabular}

top 14 proteins (1.0\% of the measured 1378) that comprise PC2 and are also differentially expressed between treatment groups include Aldh9a1, Nme2, Tfrc, Scpep1, Igkc, Fth1, Acat2, Ociad2, Ndufv2, Fabp7, Bhmt, Map1, Ephx, and Snrpd1. Notably, two of these proteins (Tfrc, and Fth1) are involved in iron acquisition and storage (Fig. 2c), which could explain the anemia patients experience post-RYGB. Furthermore, we performed Pathway Enrichment Analysis using the canonical classifications for each protein offered by KEGG, where the total number of statistically significant proteins (SSPs) classified under each pre-defined pathway was calculated. 100 pathways for Rattus norvegicus comprise at least one protein that was differentially expressed between Sham and RYGB, of which 47 comprise two or more proteins (Supplementary Table 2). This analysis provides a rudimentary assessment of which known metabolic and signaling pathways are affected by RYGB, but is subject to the inherent bias associated with pre-defining pathway boundaries.

\section{Network analysis and metabolomics integration}

For a more un-biased analysis of -omics data, we introduce a graph network-based algorithm to identify modules, or sub-networks spanning multiple canonical pathways, that are impacted by RYGB. For this analysis, the Recon 2.1 metabolic model, describing 7440 reactions in human metabolism, was downloaded from humanmetabolism.org ${ }^{21}$, and bipartite and reaction-centric graph networks were constructed as described in Supplementary Methods. Of the 1378 proteins detected in rat liver using SWATH, 1266 proteins had a corresponding gene entry catalogued in the KEGG database under the Rattus norvegicus genome and 575 possessed an enzyme commission number (EC) associated with the gene suggesting its involvement in metabolism. Of these, 346 proteins catalyze at least one of the 7440 reactions described in the Recon 2.1 network. Since a statistically significant protein (SSP) between Sham and RYGB can catalyze more than one network reaction in Recon 2.1 spanning multiple compartments, we distinguish SSPs from Statistically Significant Reactions (SSRs). For each measured protein that was a metabolic enzyme, the corresponding reactions in the Recon 2.1 metabolic network were identified based on EC numbers. For metabolomics data, collected using Multiple Reaction Monitoring (MRM) based on a protocol described by Yuan et al. ${ }^{18}, 303$ metabolites were quantified from the crushed rat liver samples. Comparing metabolite levels between Sham and RYGB, we computed the number of Statistically Significant Metabolites (SSMs, also determined using the Wilcoxon rank test with a $p$-value threshold of 0.05 and reported in Supplementary Table 3) in each KEGG pathway, a computation that is similar to the pathway enrichment tool provided by MetaboAnalyst $^{22}$. We find that pyrimidine metabolism, purine metabolism, 
a

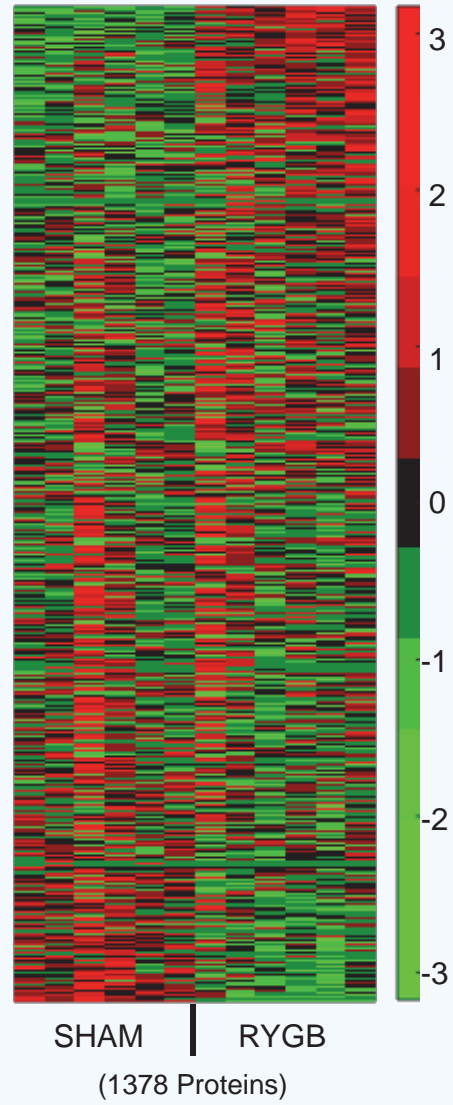

b

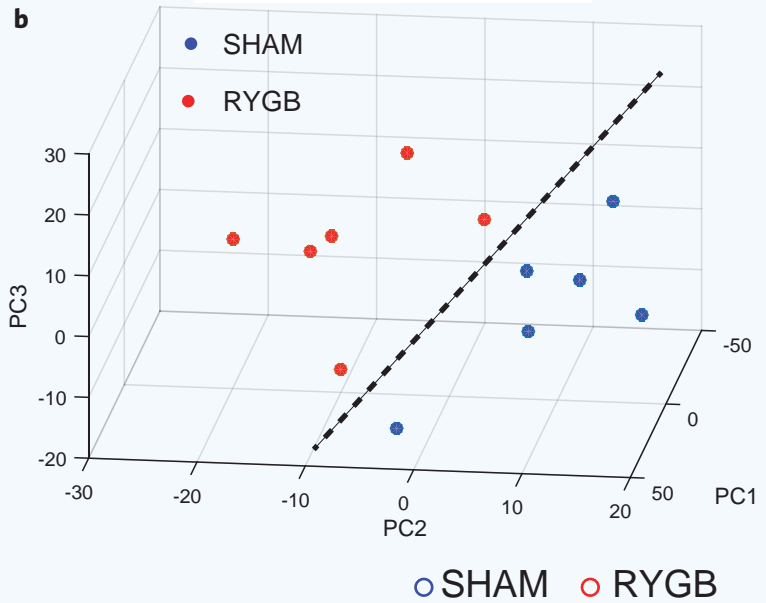

c

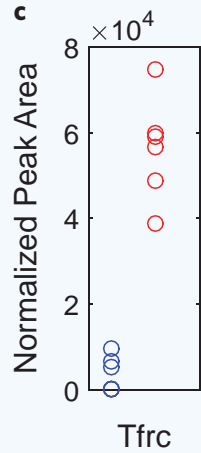

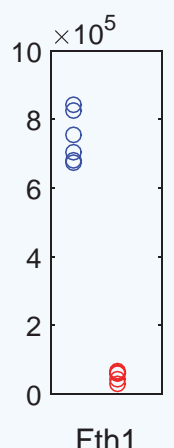

○SHAM

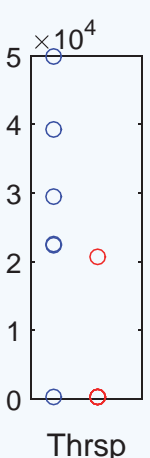

Figure 2 (a) Heatmap of SWATH Proteomics data where the relative abundance of each protein is colorcoded based on the z-score of the protein's normalized peak area. (b) Principal component analysis reveals separation between Sham and RYGB conditions (c) Selected statistically significant proteins (SSPs) highlighting perturbation to iron metabolism (Tfrc, Fth1), and potential therapeutic liver targets for obesity (Thrsp, Acot13). and glutathione metabolism were canonical pathways most enriched with SSMs, independent of proteomic information (Supplementary Table 4). Since the solvent-based metabolite extraction procedure on crushed tissue does not enable one to determine which compartment was responsible for the difference in metabolite level between Sham and RYGB, we treated all compartmental metabolites of an SSM the same. For example, taurine levels are significantly depleted in RYGB liver, but both cytosolic taurine $(\operatorname{taur}[\mathrm{c}])$ and peroxisomal taurine $(\operatorname{taur}[\mathrm{x}])$ are equally treated as SSMs in the Recon 2.1 model.

\section{Module discovery using sub-graph search}

The premise behind identifying sub-networks enriched with SSMs and SSRs is that significantly elevated or depleted biomolecules that are closely connected within a metabolic network provides evidence that RYGB is causing a perturbation to the module of reactions. Computationally, this is done by searching for random subgraphs in the Recon 2.1 reaction-centric graph of a specified size in a monte-carlo fashion and select sub-networks with a statistically higher density of SSRs and SSMs relative to the SSR/SSM density distribution for all random subgraphs. The premise of identifying these sub-networks from a more complex network of human metabolism is highlighted in Fig. 3a. The random sub-graph generation begins by starting with the seed node in the reaction-centric matrix $\mathbf{G}$ and then randomly selecting an edge connected to the seed node. The target node connected to the randomly selected edge is then added to the sub-network. Subsequently, a randomly selected node from the now two-node sub-network is chosen as a point to expand the sub-network and another randomly selected edge connecting to that node is selected in a similar manner as described above. In this regard, the sub-network grows until it reaches a specified reaction node size, in this case set to 20. The number of SSMs and SSRs in each sub-graph is then also computed.

For each of the 81 genes found to be significantly elevated or depleted between Sham and RYGB, 34 genes encode metabolic enzymes that catalyze at least one of the reactions described in the Recon 2.1 metabolic model. In total, 298 reactions in the model were catalyzed by one of the 34 metabolic genes found to be differentially expressed between the two experimental conditions. The central component of our target discovery methodology is to treat each of these reactions as a "seed" for a local network search algorithm to identify network modules with a high SSR and SSM density. The seed reaction, $R_{s}$, is used as a starting point to grow random connected sub-networks of 20 reaction nodes, which was determined to be a manageable module size for visual inspection. The distribution of maximum node-pair distance for random sub-networks of size 20 (Supplementary Fig. 6) shows that setting the module size to 20 is adequate to capture long range interactions spanning up to 9 reaction nodes. Since $99.88 \%$ of node-pair distances in $\mathbf{G}$ are less than or equal to 9 , a module size of 20 can capture the most distant interactions in the network. The Monte-Carlo sub-graph detection algorithm is allowed to run for 1 hour and the composition of each module is stored along with the number of SSRs and SSMs. The algorithm is applied on the reaction centric matrix $\mathbf{G}$ and modules enriched with SSRs and SSMs are visualized using the bipartite representation B using Cytoscape network visualization software ${ }^{23}$.

\section{Modules detected}

Several modules were discovered that exhibit regions within the metabolic network enriched with SSRs and SSMs, suggesting that the metabolic pathway captured by the module is influenced by RYGB. For example, 


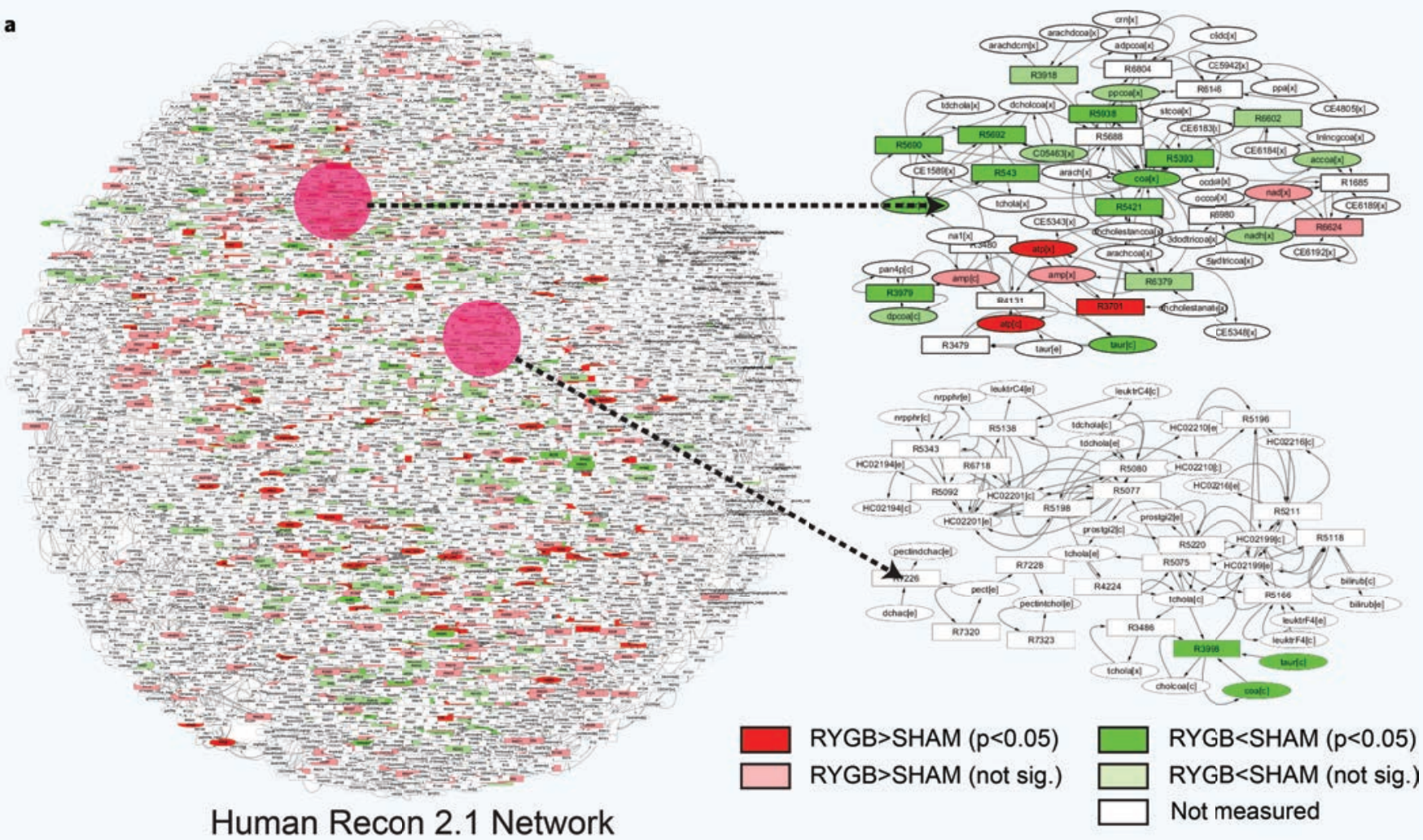

b

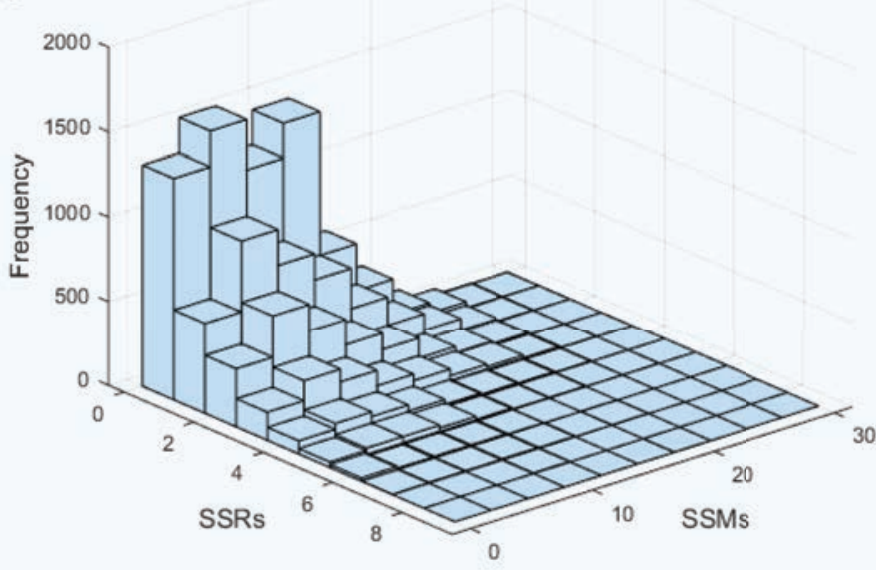

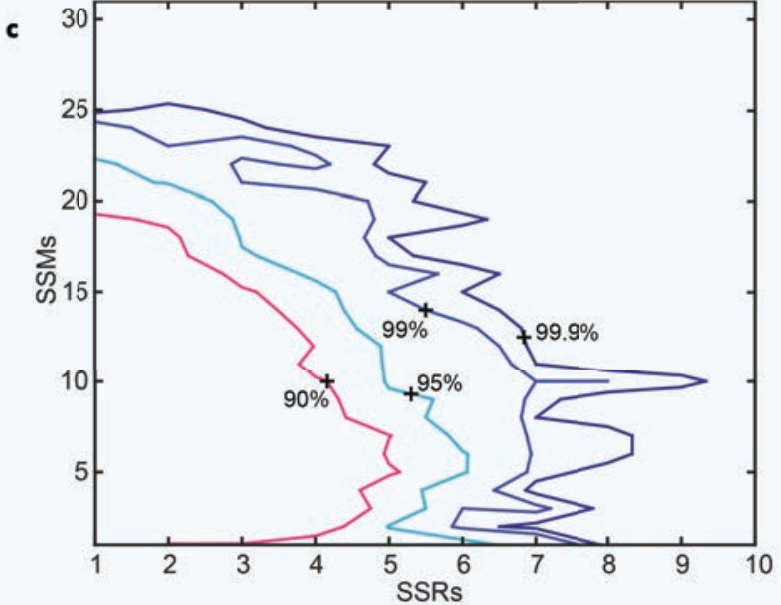

Figure 3 (a) Identifying sub-networks within a bipartite graph network of Recon 2.1 reveals modules either enriched or devoid of SSRs and SSMs. (b) Histogram for the distribution of the number of modular SSRs and SSMs and (c) the 90\%, 95\%, 99\%, and $99.9 \%$ confidence bands.

a zoomed-in module in Fig. 4a reveals the relationship between the down-regulation of Baat and the depletion of taurine, the metabolite used to conjugate $\mathrm{C} 24$ bile acids. Baat is the enzyme that catalyzes the conjugation of taurine to choloyl-coA to form taurocholate, and the connection between the significantly depleted protein and metabolite is uncovered using computational module discovery in an unbiased fashion. Similarly, the module highlighted in Fig. $\mathbf{4 b}$ reveals a perturbation to "one-carbon folate metabolism" based on the differential expression of Mthfd1 and Ftcd as well as the increase in dihydrofolate levels. The network-based modularity approach also reveals lesser intuitive cofactor-based interactions within the local network neighborhood. For example, the module featuring conjugated bile acid formation also (Fig. 4a) features the utilization CoA and other SSRs that consume or produce the cofactor. To further demonstrate the use of network-based modularity as a means to discover non-intuitive network interactions, several other modules are presented in Supplementary Materials, some of which highlight the production and consumption of NADPH, NADH, and other nucleotide triphosphates (Supplementary Fig. 1-5).

To determine if a module is significantly affected by RYGB, we compared the total SSMs and SSRs within the module to the multivariate distribution of modular SSMs and SSRs for approximately 10,000 random sub-networks of size 20 . The histogram representing this distribution 

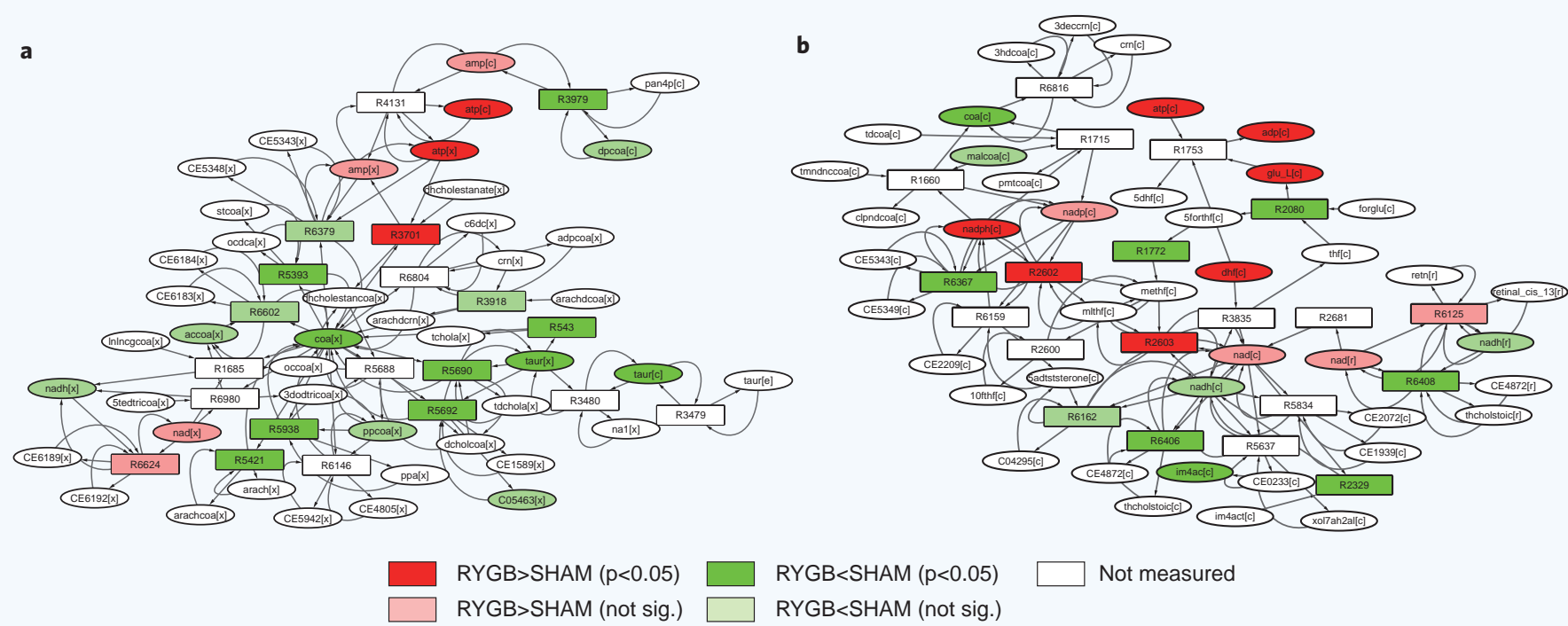

RYGB>SHAM (not sig.)

RYGB $<$ SHAM $(p<0.05)$

RYGB $<$ SHAM (not sig.)

Statistically Significant Proteins (SSPs) $\mid$ Statistically Significant Metabolites (SSMs)

Statistically Significant Proteins (SSPs) Statistically Significant Metabolites (SSMs)

\begin{tabular}{ll}
\hline Reaction & Protein \\
\hline R543 & Baat \\
R3701 & Slc27a5 \\
R3979 & Enpp3 \\
R5393 & Baat \\
R5421 & Baat \\
R5690 & Baat \\
R5692 & Baat \\
R5938 & Baat
\end{tabular}

Metabolite ID
coa $[\mathrm{x}]$
$\operatorname{taur}[\mathrm{x}]$
$\operatorname{accoa}[\mathrm{x}]$
$\operatorname{taur}[\mathrm{c}]$
$\operatorname{atp}[\mathrm{x}]$
$\operatorname{dpcoa}[\mathrm{x}]$
atp $[\mathrm{c}]$

Metabolite Name
CoenzymeA
Taurine
Acetyl-CoA
Taurine
ATP
Dephospho-CoA
ATP

\begin{tabular}{ll}
\hline Reaction & Protein \\
\hline R1772 & Ftcd \\
R2080 & Ftcd \\
R2329 & Aldh9a1, Aldh3a2 \\
R2602 & Mthfd1 \\
R2603 & Mthfd1 \\
R6367 & Cyp2a2, Cyp1a2 \\
R6406 & Aldh9a1, Aldh3a2 \\
R6408 & Aldh9a1, Aldh3a2
\end{tabular}

\begin{tabular}{ll} 
Metabolite ID & Metabolite Name \\
\hline coa[c] & CoenzymeA \\
atp $[\mathrm{x}]$ & ATP \\
adp[c] & ADP \\
glu_L[c] & L-glutamine(1-) \\
dhf[c] & 7,8 -Dihydrofolate \\
im4ac[c] & imidazol-4-ylacetate \\
nadph[c] & NADPH
\end{tabular}

Figure 4 Examples of detected modules featuring (a) Bile acid metabolism and (b) One-carbon metabolism. The SSRs and SSMs in each module are listed below the network diagrams.

(Fig. 3b) is heavily skewed where $48 \%$ of the modules contained zero SSRs. Since the distribution was non-normal, we numerically computed contour lines for the $90 \%, 95 \%, 99 \%$ and $99.9 \%$ bands (Fig. 4c) for which the specified percentage of modules lie to the left of the band. For example, the module representing bile acid conjugation (Fig. 4a) using taurine contained 8 SSPs and 7 SSMs, which falls above the 99\% band suggesting that the module has a significantly high density of SSPs and SSMs relative to randomly connected sub-networks of size 20. Indeed, the sub-network size selected and the run-time for the Monte-Carlo are both parameters that can impact the modules that are uncovered. Quantitatively, we show that there is an inverse relationship between the run-time required to identify a known set of interactions between proteins and the size the module used for the algorithm (Supplementary Fig. 7). As such, while there is a run-time benefit to picking a larger module size, visually interpreting a sub-network of several hundred nodes would be too unwieldy.

\section{DISCUSSION}

In this work, we present a novel multi-omic interrogation of liver metabolism in a rat RYGB model by integrating SWATH protoemics and MRM metabolomics data to facilitate the discovery of biochemical mechanisms associated with weight loss and achievement of glycemic control. SWATH is now the state-of-the-art label-free proteomics platform that offers unparalleled coverage of an organism's proteome owing to the dynamic range and speed offered by AB Sciex Tripletof HRMS instrumentation. However, the application of this technology to translational medicine research has been sparse and our utilization of the technique to quantify metabolic changes following RYGB in vivo using graph-based network analysis should serve as a generally applicable template for future multiomic endeavors.

Our multi-omic analysis of rat liver metabolism following 3 months of either Sham or RYGB operations revealed significant differences in protein and metabolite levels, many of which corroborate observations made in the clinic. For example, the SWATH proteomics data revealed a strong perturbation to iron storage and transport in the liver, namely that ferritin (Fth1, responsible for iron storage) levels are significantly decreased following RYGB, which is consistent with the clinical finding that patients following RYGB undergo anemia ${ }^{24}$. Intriguingly, increased serum ferritin has also been associated with insulin resistance via an inverse correlation to adiponectin levels ${ }^{25}$. In addition, our moduledetection algorithm found a perturbation to taurine-conjugated bile acid metabolism in the rat, which has also been observed in humans following RYGB. For example, Simonen et al. reported a decrease in fasting serum conjugated bile acids in RYGB patients, with an $82 \%$ reduction in taurineconjugated bile acids ${ }^{26}$. Moreover, they showed that the conjugated bile acid decrease correlated with glucose and lipid oxidation and proposed a mechanistic link to thyroid hormone regulation based on their observed increased mRNA expression of $\mathrm{DiO}_{2}$ in adipocytes. In contrast, Ahmad et al. reported post-prandial levels of taurine-conjugated bile acids in serum of lean, obese, and RYGB patients and found their levels to be higher in patients post-RYGB compared to obese patients ${ }^{27}$. Different models of gastric bypass also seem to result in a perturbation to bile acid 
metabolism, yet the trends appear opposite to what we observe following RYGB. For example, Wu et al. performed duodenal-jejunal bypass (DJB) and sleeve gastrectomy in diabetic rats and found that the expression of amino acid $\mathrm{N}$-acyltransferase $B$ aat was up-regulated with increased serum conjugated bile acid levels ${ }^{28}$. As such, further investigation is warranted to understand the dynamics of Baat expression following RYGB and how that impacts the levels of conjugated bile acids either after fasting or post-prandial states.

The ultimate translational goal of interrogating rodent RYGB models using multi-omic approaches is to discover therapeutic targets for noninvasive obesity treatments. To this end, we report Thrsp and Acot13 as two proteins down-regulated in RYGB animals (Fig. 3a), which have also been previously investigated for their impact on body weight regulation using knockout mouse models. Specifically, Kang et al. demonstrate that high fat diet fed Them $2^{-l-}$ mice exhibited a $10 \%$ reduced body weight compared to high fat diet fed Them $2^{+/+}$mice $^{29}$ and Anderson et al. showed a $26 / 29$ (male/female) \% reduced body weight gain in a $11 \%$ fat by weight diet for Thrsp knockout mice ${ }^{30}$. As a result, these targets may prove to be promising targets for hepatic knockdown using siRNA. However, further LOF/GOF experiments are required to determine if the down-regulation of these proteins can modulate body weight or if the observed protein expression following RYGB is instead a consequence of a more complex regulatory mechanism.

The module detection algorithm that was developed for this study provides an elegant integration of proteomics and metabolomics data to identify which metabolic pathways are impacted by the experimental perturbation. We find that modularity analysis can be advantageous to conventional pathway enrichment analysis (PEA) in uncovering lesserintuitive interactions between statistically significant components. For example, conventional pathway analysis would have identified two SSPs, Ftcd and Mthfd1, as both belonging to "One carbon folate metabolism" (Supplementary Table 2). However, modularity analysis also showed that the SSP Cyp2a2, involved in detoxification, could also be connected to the pathway based on the shared consumption of NADPH (Fig. 4b). Network analysis also provides a framework for visually presenting the relationship between metabolomics and proteomics data in a way that would be challenging with PEA. For example, PEA on metabolomics data would have revealed taurine as an SSM involved in "Primary bile acid biosynthesis"; however, the relationship between taurine and the SSP enzyme Baat (Fig. 4a) may not be intuitive to a non-biochemist. Modularity analysis using metabolic networks precludes an additional manual curation step to piece together relationships between metabolites and proteins that would be required if only using conventional PEA methods.

One major challenge in interpreting the uncovered metabolic modules data lies in ascribing directional changes, or determining the up/down regulation of a set of closely related genes that resulted from the experimental condition. For example, the module capturing reactions from folate metabolism features increased levels of Mthfd 1 and dihydrofolate in RYGB compared to Sham, serving as multi-omic evidence that a tetrahydrovolate interconversion module is up-regulated (Fig. 4 b). However, the decrease in Ftcd, which serves to replenish tetrahydrofolate from formiminoglutamate, obfuscates such a conclusion from being made without further experimentation. In this regard, the interpretation of modules enriched in SSRs and SSMs is limited to identifying perturbed regions of metabolic networks, and drawing conclusions about whole pathway directional changes is oftentimes a stretch due to conflicting evidence from multi-omic data. In another example, we find that while coenzyme A levels are decreased due to RYGB, two enzymes catalyzing reactions producing coA (Baat, Acat2) are differentially expressed in opposite directions (Supplementary Fig. 2), suggesting that the levels of proteins that consume coA are not driven solely by the metabolite pool.

The research workflow presented here, demonstrating a powerful multi-omic interrogation of rodent RYGB, will surely pave the way for impactful future investigations. For example, in this study, we only performed the -omics analysis on a single time point, three months post-RYGB in just liver. A natural extension of this work will be to perform a time-course multi-omic analysis to study the dynamics of metabolic reprogramming in multiple organs (e.g., adipose tissue, small intestine, and muscle), to better capture the molecular underpinnings of the therapeutic benefits of the surgery. In addition, SWATH-based proteomics analysis of plasma samples and causal network analysis ${ }^{31}$ could enable the discovery of novel differentially expressed metabolites or proteins that may drive the observed perturbations to tissue-specific metabolic modules post-RYGB. Another angle to consider is the impact of the gut-microbiome compositional shifts following RYGB on metabolic outcomes. For example, Liou et al. demonstrated that RYGB in mice causes a significant shift in the gut-microbiome population, and that fecal transplants of RYGB treated animals into lean germ-free animals elicits a $5.0 \%$ body weight reduction ${ }^{11}$. In this light, further examination of the gut-liver axis holds promise to discover microbial products regulate liver metabolism. Indeed, we also identify indole-3-carboxylic acid and phenylpyruvate to be significantly elevated RYGB liver extracts (Supplementary Table 2), both of which have previously been identified to be gut-microbiome derived using computational pathway analysis ${ }^{32}$.

In conclusion, we have demonstrated in this study a novel experimental and computational workflow as a technological advancement for the study of metabolic disease using a combination of rodent RYGB models and multi-omics approaches. We have applied this platform to interrogate the metabolic pathways that are altered in the liver following RYGB in rats, and identified several targets that may play a role in the regulation of hepatic energy homeostasis following the surgery. We expect that the combined computational and experimental framework presented here will further facilitate systematic investigations into the mechanisms that mediate the therapeutic benefits of the surgery, and ultimately, the discovery of novel therapeutics for the metabolic syndrome.

\section{REFERENCES}

1. Yang, L. \& Colditz, G.A. Prevalence of overweight and obesity in the United States, 2007-2012. JAMA Intern. Med. 295, 1549-1555 (2015).

2. Chan, E.W. et al. Efficacy and safety of lorcaserin in obese adults: A meta-analysis of 1-year randomized controlled trials (RCTs) and narrative review on short-term RCTs. Obes. Rev. 14, 383-392 (2013).

3. Korner, J. et al. Effects of Roux-en-Y gastric bypass surgery on fasting and postprandial concentrations of plasma ghrelin, peptide YY, and insulin. J. Clin. Endocrinol. Metab. 90, 359-365 (2005).

4. Campbell, L.E. et al. Identification of novel changes in human skeletal muscle proteome after Roux-en-Y gastric bypass surgery. Diabetes 65, 2724-2731 (2016).

5. Seyfried, F. et al. Urinary phenotyping indicates weight loss-independent metabolic effects of roux-en-Y gastric bypass in mice. J. Proteome Res. 12, 1245-1253 (2013).

6. Arora, T. et al. Roux-en-Y gastric bypass surgery induces early plasma metabolomic and lipidomic alterations in humans associated with diabetes remission. PLoS One 10. e0126401 (2015).

7. Luo, P. et al. Metabolomics study of Roux-en-Y gastric bypass surgery (RYGB) to treat type 2 diabetes patients based on ultraperformance liquid chromatography-mass spectrometry. J. Proteome Res. 15, 1288-1299 (2016).

8. Yarmush, M.L., D'Alessandro, M. \& Saeidi, N. Regulation of energy homeostasis after gastric bypass surgery. Annu. Rev. Biomed. Eng. 19, 459-484 (2017).

9. Bruinsma, B.G., Uygun, K., Yarmush, M.L. \& Saeidi, N. Surgical models of Roux-en-Y gastric bypass surgery and sleeve gastrectomy in rats and mice. Nat. Protoc. 10, 495-507 (2015).

10. Saeidi, N. et al. Reprogramming of intestinal glucose metabolism and glycemic control in rats after gastric bypass. Science 341, 406-410 (2013).

11. Liou, A.P. et al. Conserved shifts in the gut microbiota due to gastric bypass reduce host weight and adiposity. Sci. Transl. Med. 5, 178ra41 (2013).

12. Yang, Z.-X., Shen, W. \& Sun, H. Effects of nuclear receptor FXR on the regulation of liver lipid metabolism in patients with non-alcoholic fatty liver disease. Hepatol. Int. 4, 741-748 (2010)

13. Arnhard, K., Gottschall, A., Pitterl, F. \& Oberacher, H. Applying 'Sequential Windowed Acquisition of All Theoretical Fragment Ion Mass Spectra' (SWATH) for systematic toxicological analysis with liquid chromatography-high-resolution tandem mass spectrometry. Anal. Bioanal. Chem. 407, 405-414 (2015). 
14. Huang, Q. et al. SWATH enables precise label-free quantification on proteome scale. Proteomics 15, 1215-1223 (2015).

15. Aebersold, R. \& Mann, M. Mass-spectrometric exploration of proteome structure and function. Nature 537, 347-355 (2016).

16. Gao, Y. et al. Quantitative proteomics by SWATH-MS reveals sophisticated metabolic reprogramming in hepatocellular carcinoma tissues. Sci. Rep. 7, 45913 (2017).

17. Rosenberger, G. et al. A repository of assays to quantify 10,000 human proteins by SWATH-MS. Sci. Data 1, 140031 (2014).

18. Yuan, M., Breitkopf, S.B., Yang, X. \& Asara, J.M. A positive/negative ion-switching, targeted mass spectrometry-based metabolomics platform for bodily fluids, cells, and fresh and fixed tissue. Nat. Protoc. 7, 872-881 (2012).

19. Shin, A.C., Zheng, H., Pistell, P.J. \& Berthoud, H.-R. Roux-en-Y gastric bypass surgery changes food reward in rats. Int. J. Obes. (Lond.) 35, 642-651 (2011).

20. Kanehisa, M. \& Goto, S. Kyoto encyclopedia of genes and genomes. Nucleic Acids Res. 28, 27-30 (2000).

21. Thiele, I. et al. A community-driven global reconstruction of human metabolism. Nat Biotechnol. 31, 419-425 (2013).

22. Xia, J., Sinelnikov, I.V., Han, B. \& Wishart, D.S. MetaboAnalyst 3.0-making metabolomics more meaningful. Nucleic Acids Res. 43, W251-W257 (2015).

23. Shannon, P. et al. Cytoscape: A software environment for integrated models of biomolecular interaction networks. Genome Res. 13, 2498-2504 (2003).

24. Vargas-Ruiz, A.G., Hernández-Rivera, G. \& Herrera, M.F. Prevalence of iron, folate, and vitamin B12 deficiency anemia after laparoscopic Roux-en-Y gastric bypass. Obes. Surg. 18, 288-293 (2008).

25. Gabrielsen, J.S. et al. Adipocyte iron regulates adiponectin and insulin sensitivity. J. Clin. Invest. 122, 3529-3540 (2012).

26. Simonen, M. et al. Conjugated bile acids associate with altered rates of glucose and lipid oxidation after Roux-en-Y gastric bypass. Obes. Surg. 22, 1473-1480 (2012)

27. Ahmad, N.N., Pfalzer, A. \& Kaplan, L.M. Roux-en-Y gastric bypass normalizes the blunted postprandial bile acid excursion associated with obesity. Int. J. Obes. (Lond.) 37, 1553-1559 (2013)

28. Wu, Q. et al. Effects of bariatric surgery on serum bile acid composition and conjugation in a diabetic rat model. Obes. Surg. 26, 2384-2392 (2016).

29. Kang, H.W., Niepel, M.W., Han, S., Kawano, Y. \& Cohen, D.E. Thioesterase superfamily member 2/acyl-CoA thioesterase 13 (Them2/Acot13) regulates hepatic lipid and glucose metabolism. FASEB J. 26, 2209-2221 (2012).

30. Anderson, G.W. et al. The Thrsp null mouse (Thrsptm1cnm) and diet-induced obesity. Mol. Cell. Endocrinol. 302, 99-107 (2009).

31. Krämer, A. et al. Causal analysis approaches in Ingenuity Pathway Analysis. Bioinformatics 30, 523-530 (2014).

32. Sridharan, G.V. et al. Prediction and quantification of bioactive microbiota metabolites in the mouse gut. Nat. Commun. 5, 5492 (2014).

\section{SUPPLEMENTARY INFORMATION}

\section{Sections}

Supplementary Methods

Statistically Significant Proteins and Descriptions

Pathway Enrichment Analysis for SSPs

Metabolomics (Individual Metabolite Figures)

Pathway Enrichment Analysis for SSMs

Coverage of SWATH for Rat Liver Proteomics

Modules Relating SSRs and SSMs

Maximum Module Shortest Path Figure

Supplementary References

\section{SUPPLEMENTARY METHODS}

\section{SWATH proteomics}

Proteomics data using SWATH acquisition was collected on tissue biopsy (3 months post-surgery) protein extracts. Each biopsy was ground into powder using a mortar and pestle in liquid nitrogen, and then reconstituted in $7 \mathrm{M}$ urea, $2 \mathrm{M}$ thiourea, $4 \%$ CHAPS, reduced and alkylated. Proteins were then precipitated with six volumes of acetone overnight after which they were washed twice with methanol: $41 \mathrm{~g}$ of each of the six RYGB and six Sham samples were pooled and digested onpellet with $2 \times 3 \mathrm{~g}$ trypsin in $50 \mathrm{mM}$ Tris $\mathrm{pH} 8+0.1 \%$ NaDOC. Peptides were fractionated into six fractions using increasing percentage of acetonitrile on a reverse phase solid phase extraction (SPE) column. The individual samples were treated identically, but were eluted with $75 \%$ acetonitrile. Each of the 12 fractions and 12 samples were then analyzed using LC-MS/MS. Acquisition was performed with a 5600 TripleTOF LC/MS-MS (AB Sciex, Foster City, CA) equipped with an electrospray interference with a $25 \mathrm{~m}$ iD capillary and coupled to an Eksigent UHPLC (Eksigent, Redwood City, CA). The acquisition and data processing was controlled using Analyst TF 1.6 software. The source voltage was set to $5.2 \mathrm{kV}$ and maintained at $225^{\circ} \mathrm{C}$, curtain gas was set as $27 \mathrm{psi}$, gas 1 at $12 \mathrm{psi}$, and gas 2 at $10 \mathrm{psi}$. Acquisition was performed in Information Dependent Acquisition (IDA) for the 2D protein database and in SWATH acquisition mode for the samples. Separation was performed on a reverse phase HALO C18-ES column $0.3 \mu \mathrm{m}$ i.d., $2.7 \mu \mathrm{m}$ particles, $150 \mathrm{~mm}$ long (Advance Materials Technology, Wilmington, DE) which was maintained at $60^{\circ} \mathrm{C}$. Samples were injected by loop overfilling into a $5 \mu \mathrm{L}$ loop. For the 60 minute (2D database) or the 120 minute (samples) LC gradient, the mobile phase consistent of solvent A $(0.2 \% \mathrm{v} / \mathrm{v}$ formic acid and $3 \% \mathrm{DMSO} v / \mathrm{v}$ in water $)$ and solvent $\mathrm{B}(0.2 \% \mathrm{v} / \mathrm{v}$ formic acid and $3 \%$ DMSO in EtOH) at a flow rate of $3 \mu \mathrm{L} / \mathrm{min}$. Protein identification was performed with ProteinPilot V4.5 (AB Sciex) with the instrument pre-set for the AB Sciex 5600 TripleTOF and Iodoacetamide as cys alkylation as special factor.

\section{Recon 2.1 network preparation}

The Recon metabolic network was downloaded from humanmetabolism. org in a Matlab compatible .mat file, which comprised of a struct with reaction definitions, metabolite names with KEGG identification numbers, and a stoichiometric matrix. We chose to work with a human metabolic model since our findings in rat are only significant if applicable to target discovery for humans. Using this model, a bipartite graph network of both reaction and metabolite nodes was constructed in the form of a square connectivity matrix (B) of size $N+M$, where $N$ is the number of reactions and $M$ is the number of metabolites in the model respectively. With reactions listed before metabolites, the $i$ th metabolite was assigned to the $(N+i)$ th row and column in $\mathbf{B}$. A directed edge was assigned from a reaction node $\left(R_{i}\right)$ to a metabolite node $\left(M_{i}\right)$ if $M_{i}$ is produced by the reaction $R_{i}$ and is described by assigning a ' 1 ' to the entry $\mathbf{B}(i, N+i)$. Similarly, a directed edge was assigned from a $M_{i}$ to $R_{i}$ if the metabolite $M_{i}$ is consumed by the reaction $R_{i}$ and an entry ' 1 ' is assigned to $\mathbf{B}(N+$ $i, i$ ). All other matrix entries in $\mathbf{B}$ are otherwise assigned a value of ' 0 '. If a reaction was assigned as reversible, both directions were considered for edge assignment. In this study, we included edges mediated through the production and consumption of metabolic cofactors such as ATP, NADH, NADPH, but excluded meaningless interactions based on inorganic ions such as $\mathrm{H}^{+}$. A complete list of which metabolites were considered for network edges is provided in Supplementary Data 1. From the bipartite representation of Recon 2, an NXN reaction-centric graph $(\mathbf{G})$ was also constructed to enable module detection based on enzymes while metabolites are treated as a shared resource. In this case, an undirected edge between a reaction pair $\left[R_{i}, R_{j}\right]$ was assigned $(\mathbf{G}(i, j)=1)$ if a metabolite produced by $R_{i}$ is consumed by $R_{j}$. 
Supplementary Table 1 Significantly elevated or depleted liver proteins between Sham and RYGB.

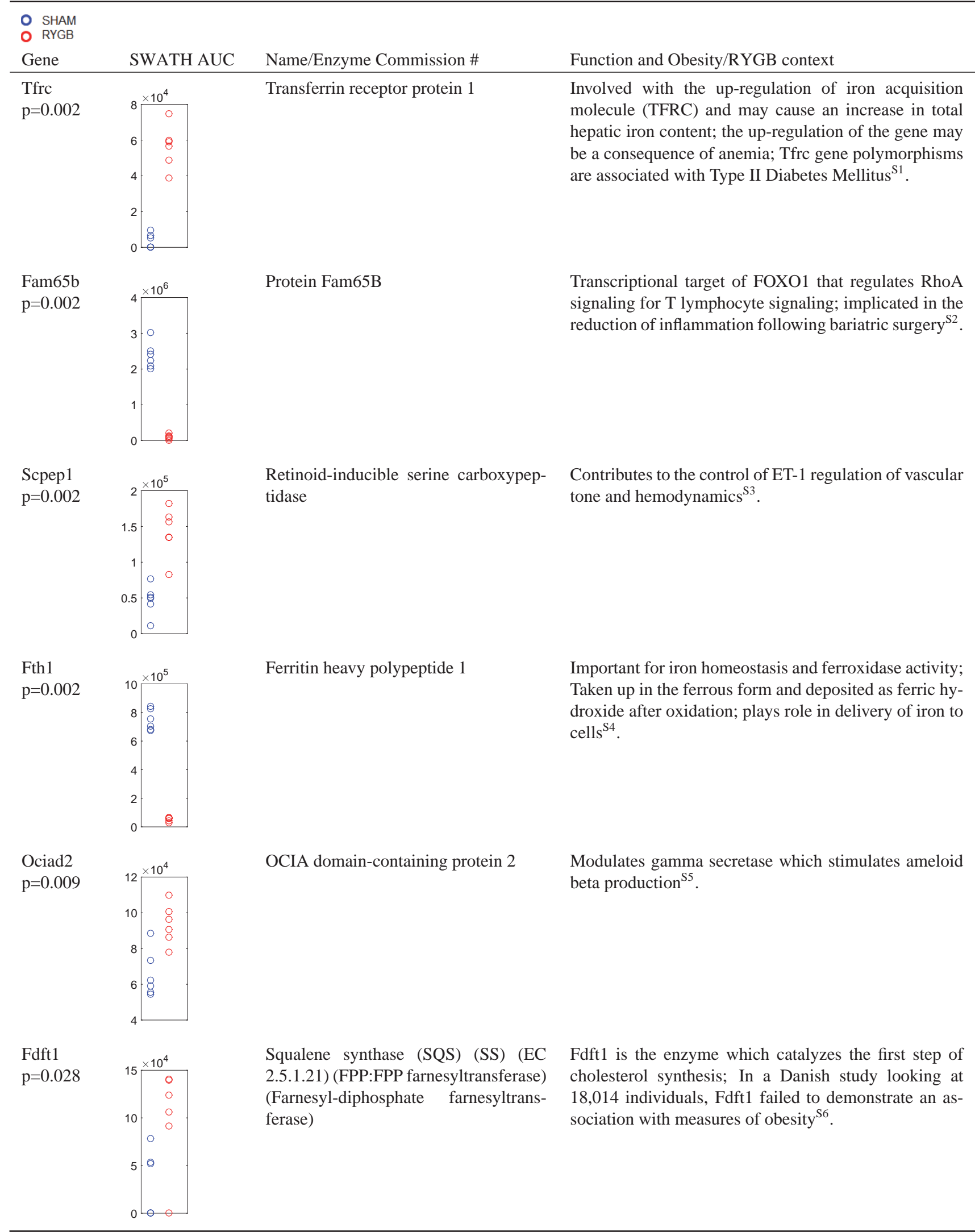

(Continued) 
Supplementary Table 1 (Continued)

\begin{tabular}{|c|c|c|}
\hline $\begin{array}{ll}\text { O SHAM } \\
\text { O RYGB }\end{array}$ & & \\
\hline Gene & & WAT \\
\hline 7. Cpq & & $\times 10^{6}$ \\
\hline & & $\stackrel{\circ}{\circ}$ \\
\hline & 1.5 & 0 \\
\hline & 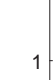 & $\begin{array}{ll}\circ & 0 \\
8 & \circ \\
8 & 0\end{array}$ \\
\hline & & \\
\hline
\end{tabular}

Asl $\mathrm{p}=0.009$

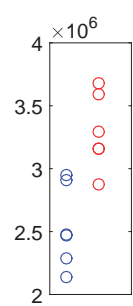

Glb1

$\mathrm{p}=0.022$

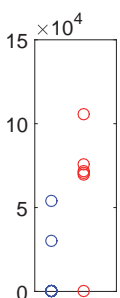

Ebp

$\mathrm{p}=0.041$

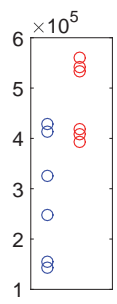

A1i3 $\mathrm{p}=0.015$

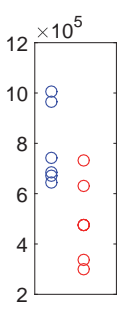

Ftcd $\mathrm{p}=0.015$

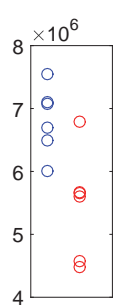

Name/Enzyme Commission \#

Carboxypeptidase Q

Argininosuccinate lyase (ASAL) (EC 4.3.2.1) (Arginosuccinase)

No Gene Name

3- $\beta$-hydroxysteroid-Delta(8),Delta(7)isomerase

Alpha-1-inhibitor-3

Formimidoyltransferase-

cyclodeaminase
Function and Obesity/RYGB context

Negative regulator of adipocyte differentiation; knockdown of Cpq in mature adipocytes leads to increased pro-adipogenic capacity of secreted fraction, implying this factor repreesnts inhibitory molecules derived from mature adipocytes ${ }^{\mathrm{S} 7}$.

Catalyzes fourth reaction in urea cycle, allows for breakdown of argininosuccinic acid to arginine and fumarate; involved in synthesis of arginine from ornithine and carbamoyl phosphate ${ }^{\mathrm{S} 8}$.

hydrolyzes $\beta$-galactose from glycoconjugates and is the origin of senescence-associated $\beta$-gal activity; Mutations in Glbl lead to deficiency in the activity of lysosomal $\beta$-galactosidase lipid accumulation ${ }^{\mathrm{S} 9}$.

Enzyme involved with catalyzing an intermediate step in the conversion of lanosterol to cholesterol ${ }^{\mathrm{S} 10}$.

Member of the macroglobulin superfamily of protease inhibitors; A negative acute phase protein which decreases significantly during an acute phase response; Studies have postulated that decreased levels of A1I3 in the blood would increase the activities of proteases causing non-specific breakdown of proteins and damage of intercellular tissue which would lead to loss of body weight ${ }^{\mathrm{S} 11}$.

Involved in folate metabolism (THF); The gene encodes the enzyme formiminotransferase cyclodeaminase which is a metabolism enzyme that links histidine catabolism to folate metabolism $^{\mathrm{S} 12}$. 
Supplementary Table 1 (Continued)

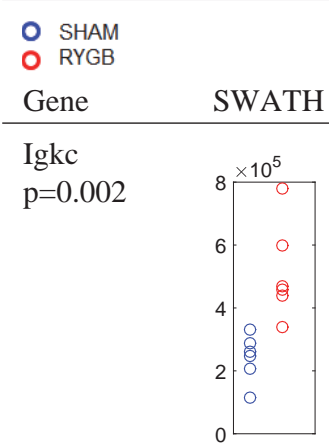

Plin2

$\mathrm{p}=0.013$

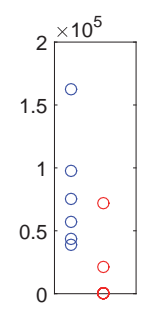

Aass

$\mathrm{p}=0.002$

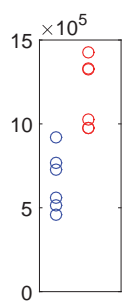

Acat2

$\mathrm{p}=0.004$

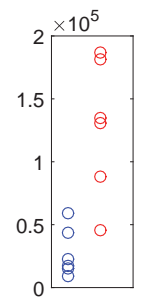

Tymp

$\mathrm{p}=0.026$

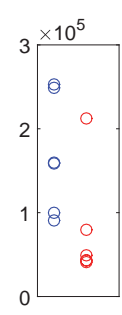

Fabp7

$\mathrm{p}=0.015$
Name/Enzyme Commission \#

Immunoglobulin Kappa Constant Region

Perilipin 2

Alpha-aminoadipic semialdehyde synthase, mitochondrial

Acetyl-coA acetyltransferase 2

Thymidine phosphorylase

Fatty acid binding protein 7
Function and Obesity/RYGB context

Strong prognostic marker in several human solid tumors, including breast cancer; mechanism is not entirely known ${ }^{\mathrm{S} 13}$.

Increased expression Plin2 leads to accumulation of cellular lipids; Overexpression of the gene affects enzymes involved in lipolysis and lipogenesis; Cells overexpressing Plin2 had elevated levels of triacylglycerols, fatty acids, cholesteryl esters, and phospholipids; Studies have shown that Plin2 deficiency results in reduced lipid droplet size and number ${ }^{\mathrm{S} 14}$.

Involved in lysine metabolism; Saccharopine in the pathway is oxidized to $\alpha$-aminoadipic semialdehyde and glutamate by saccharopine dehydrogenase ${ }^{\mathrm{S} 15}$.

Involved in cholesterol metabolism, catalyzing the conversion of acetyl-CoA to acetoacetyl-coA; ACAT2selective inhibitors have been associated with effective cholesterol-lowering or anti-atherosclerotic agents $\mathrm{S} 16$.

Encodes for a growth factor involved in cell proliferation and angiogenesis; Involved in nucleotide synthesis; Catalyzes the reversible phosphorolysis of thymidine which regulates the amount of nucleosides in cells necessary to maintain a balanced pool of deoxyribonucleoside triphosphate for replication and repair of $\mathrm{DNA}^{\mathrm{S} 17}$.

Associated with fatty acid metabolism; Involved in hepatic steatosis $^{\mathrm{S} 18}$. 
Supplementary Table 1 (Continued)

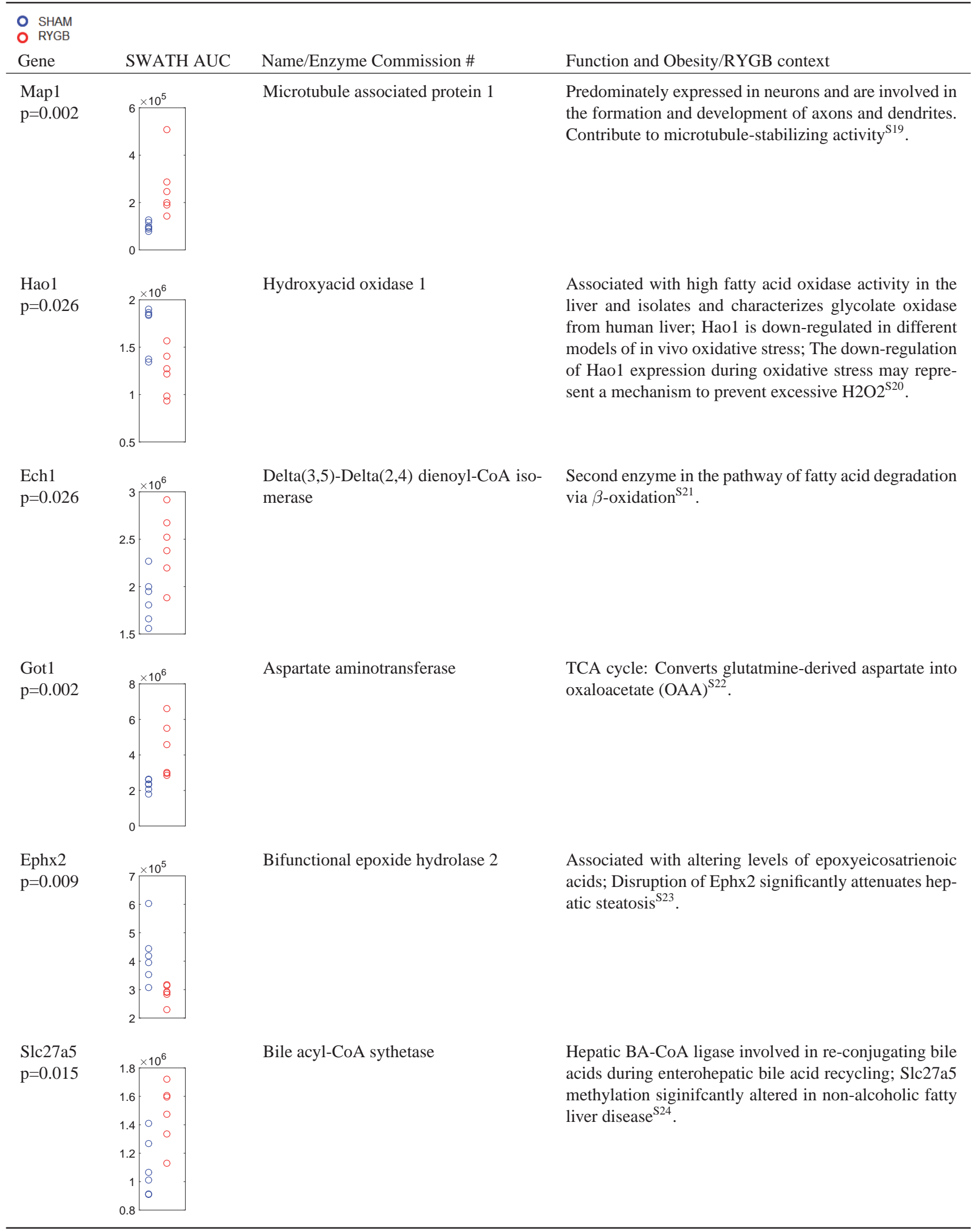


Supplementary Table 1 (Continued)

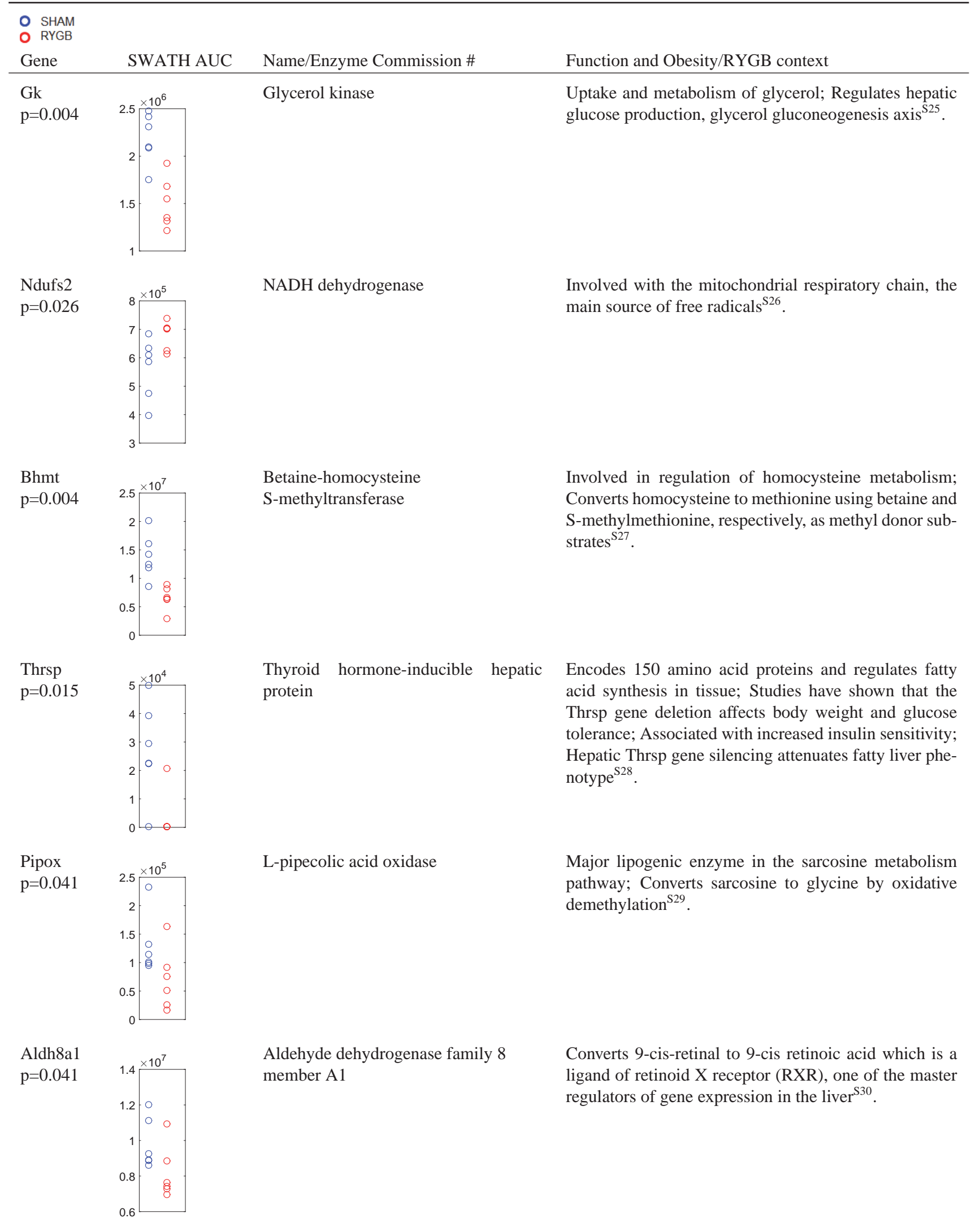

(Continued) 
Supplementary Table 1 (Continued)

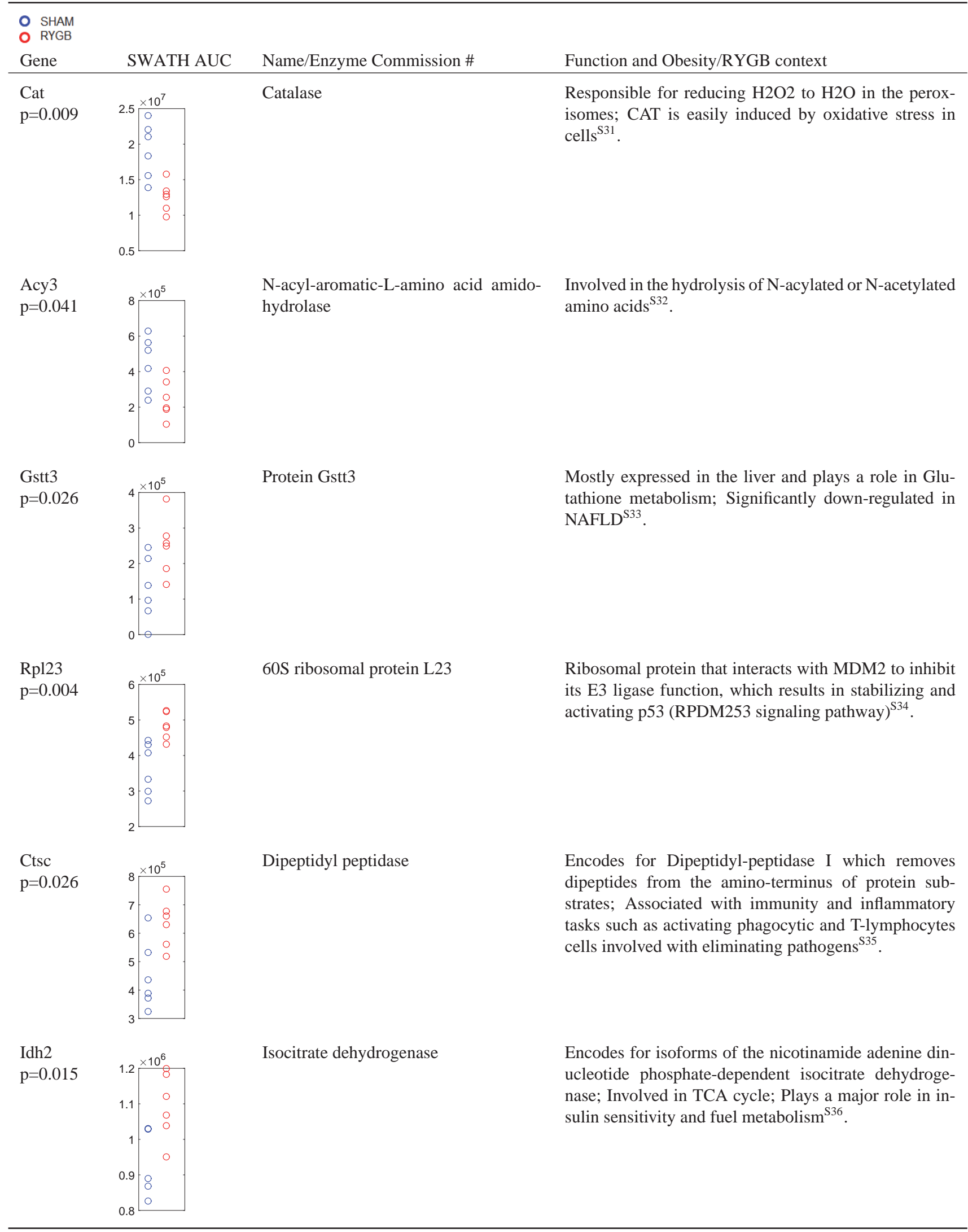


Supplementary Table 1 (Continued)

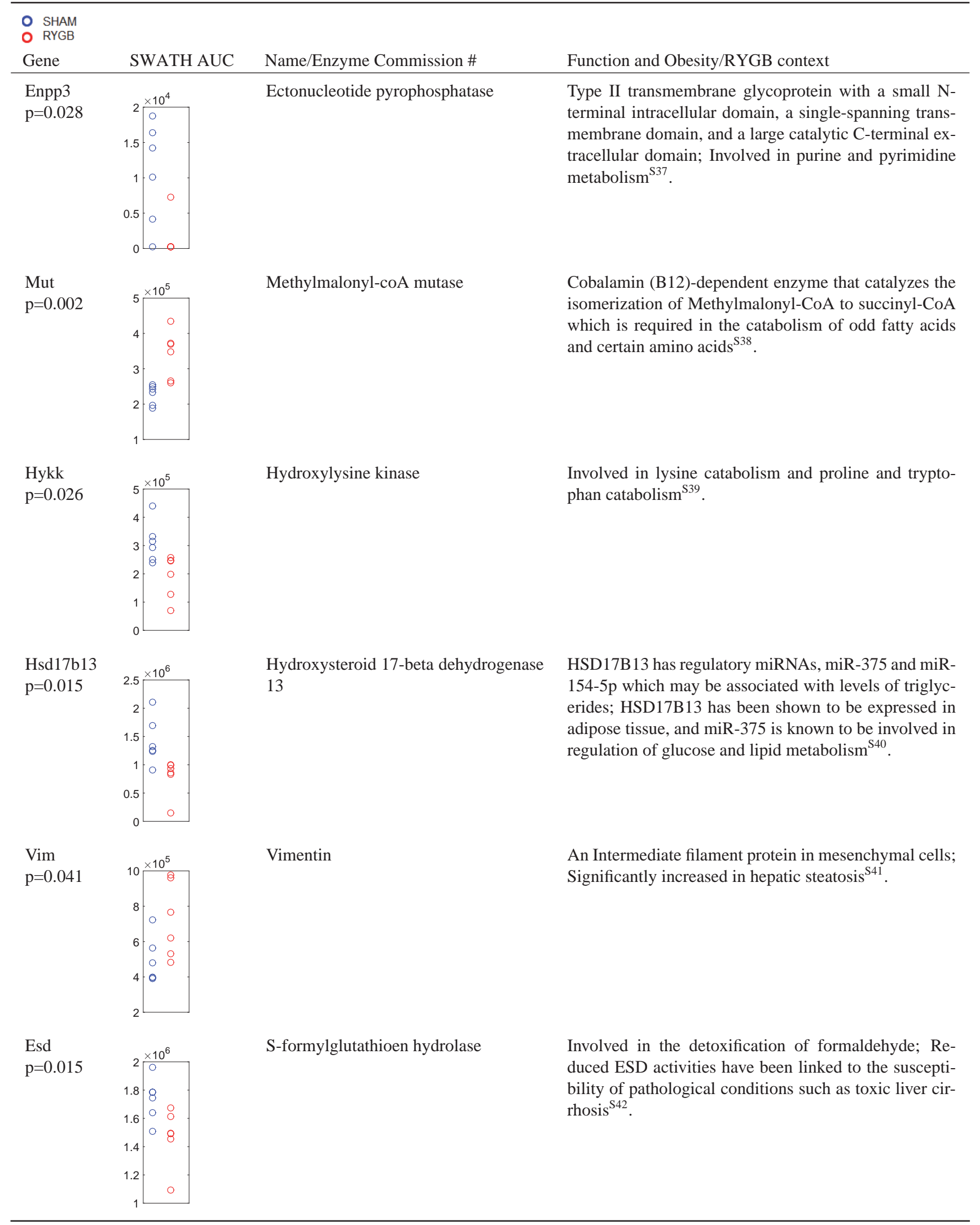

(Continued) 
Supplementary Table 1 (Continued)

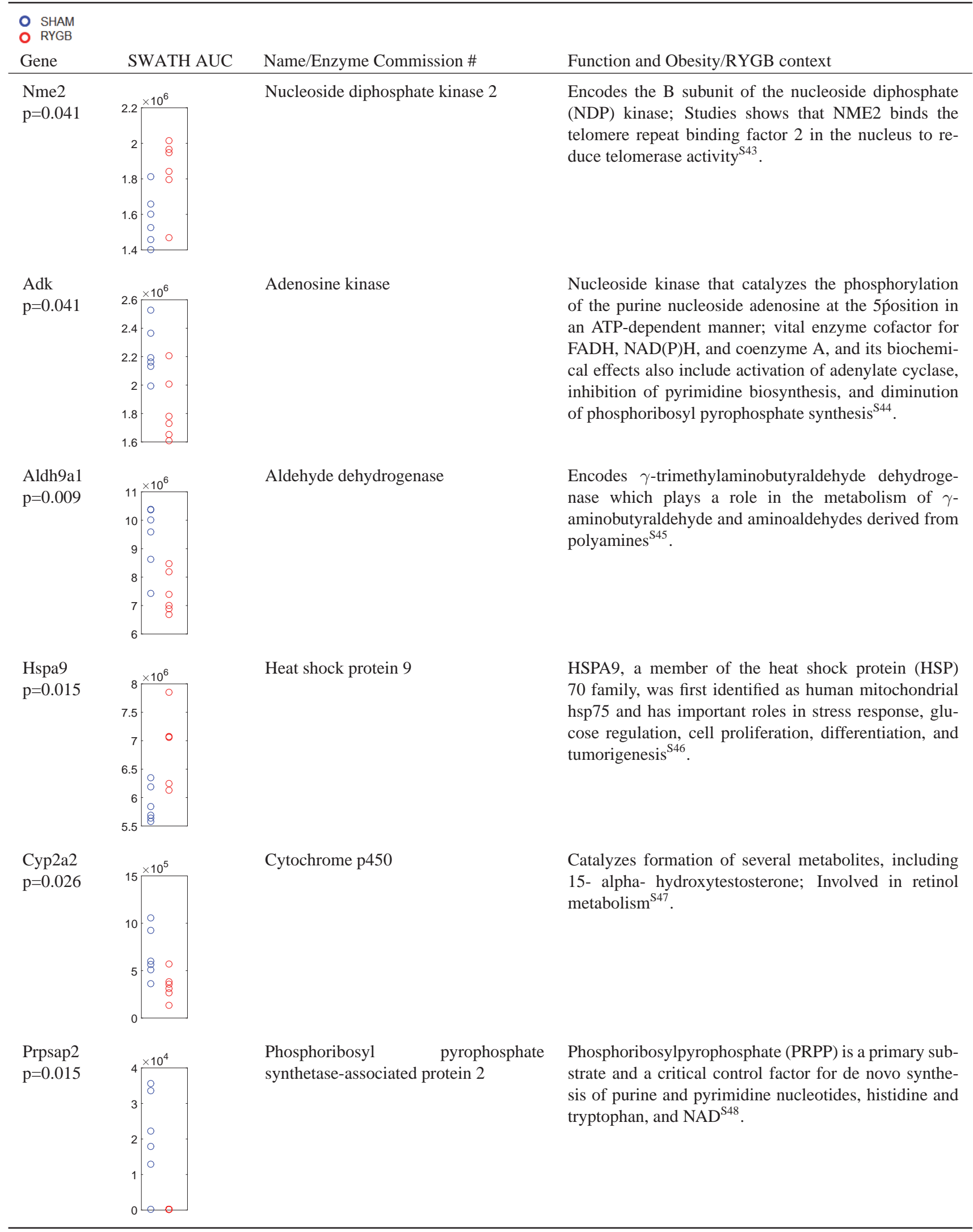


Supplementary Table 1 (Continued)

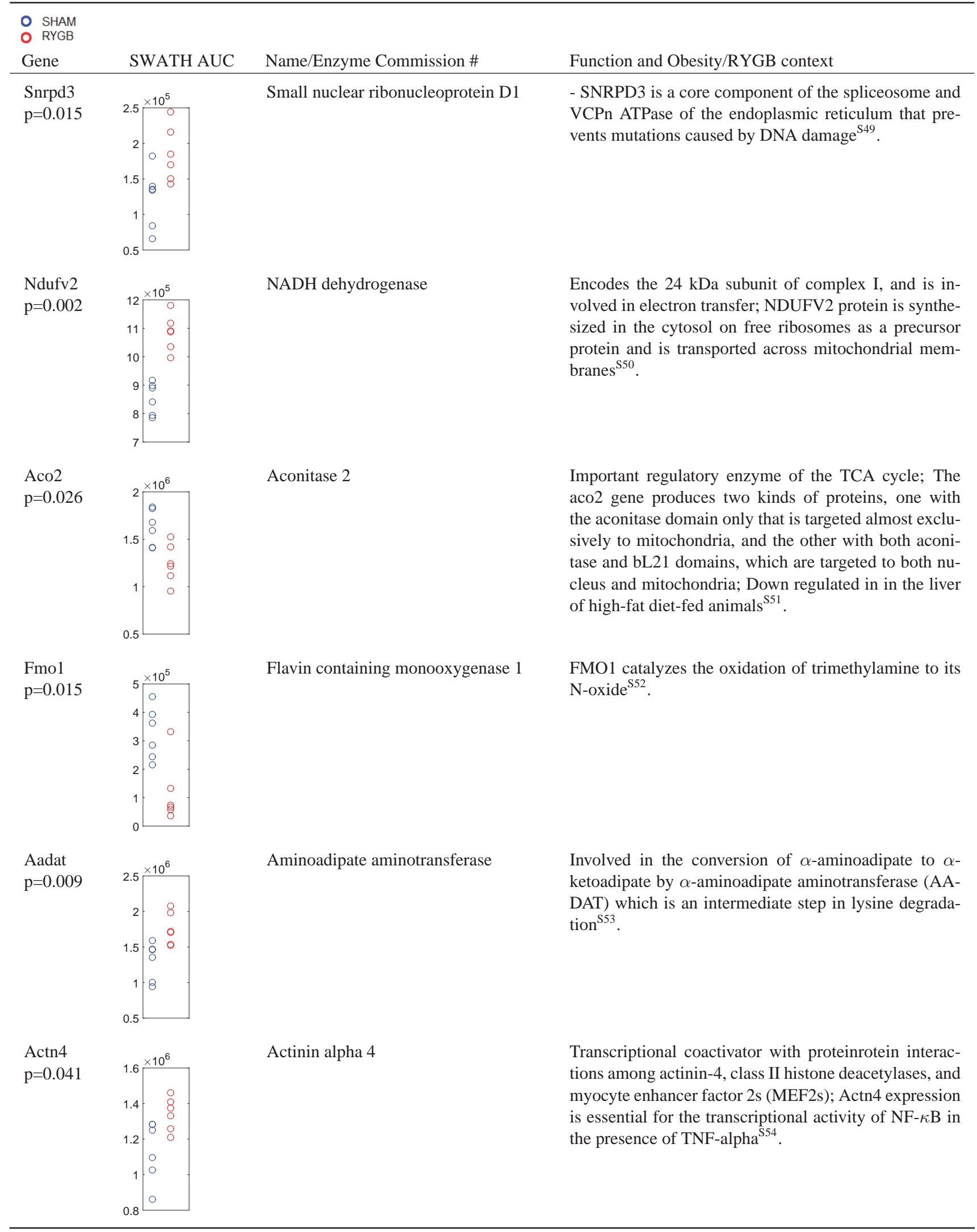

(Continued) 
Supplementary Table 1 (Continued)

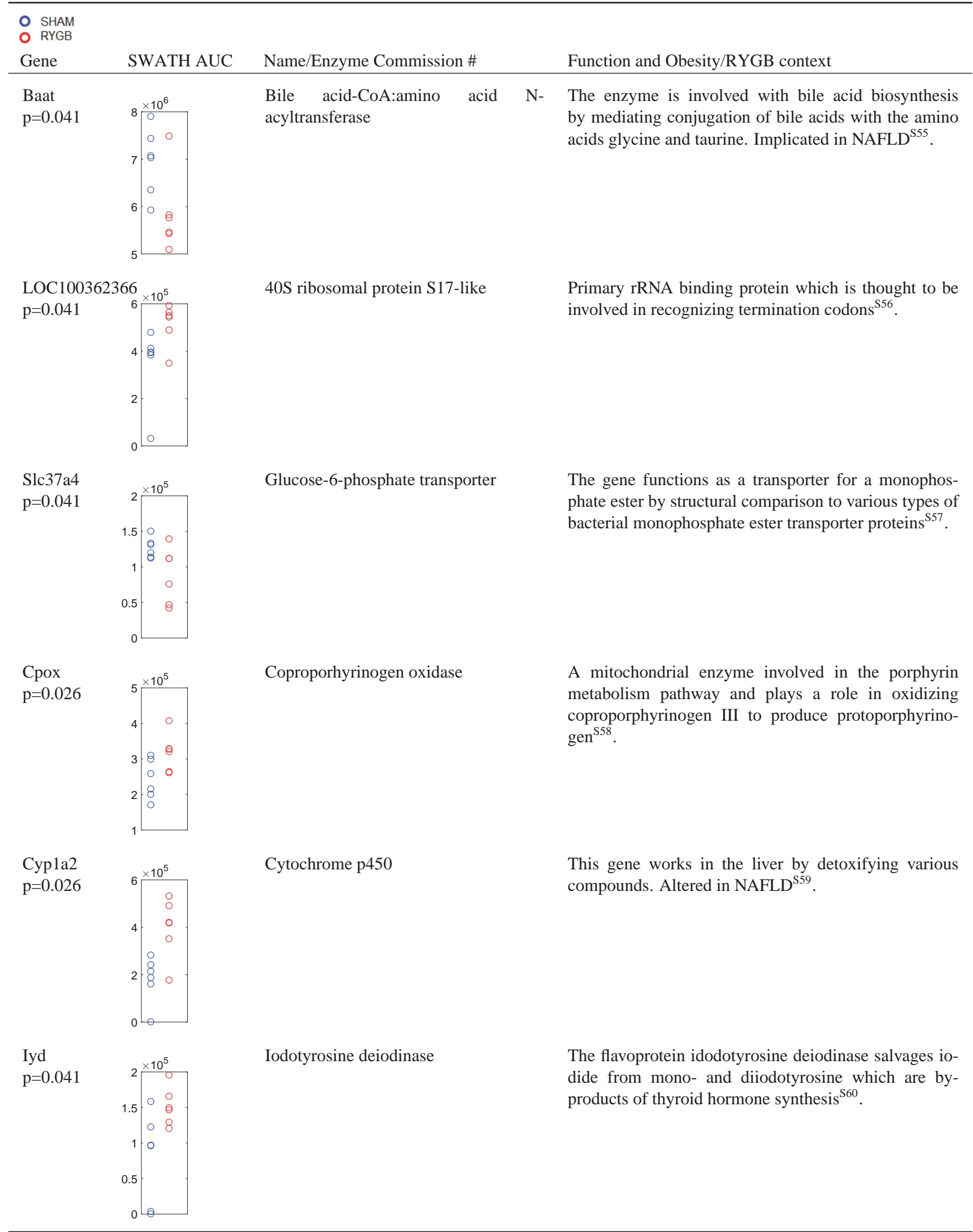


Supplementary Table 1 (Continued)

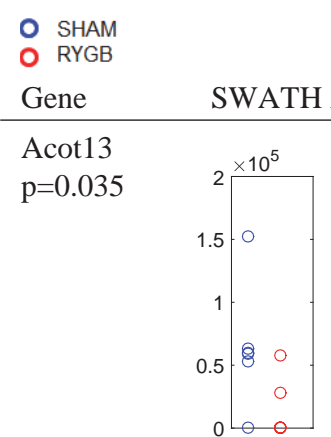

Ywhab $\mathrm{p}=0.035$

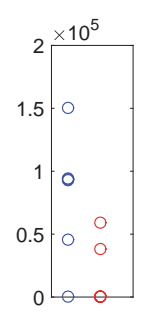

Gss $\mathrm{p}=0.041$

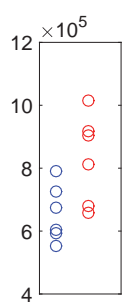

Gys2 $\mathrm{p}=0.009$

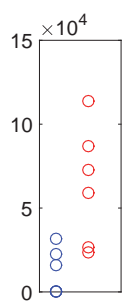

Adhfe 1
$\mathrm{p}=0.026$

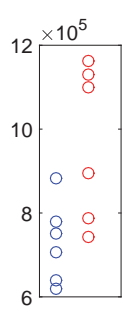

Aldh3a2 $\mathrm{p}=0.026$
Name/Enzyme Commission \#

Acyl-CoA thioesterase 13

Tyrosine 3-monooxygenase/tryptophan 5-monooxygenase activation protein, theta

Glutathione synthetase

Glycogen synthase 2

Hydroxyacid-oxoacid transhydrogenase

Aldehyde dehydrogenase
Function and Obesity/RYGB context

The enzyme is involved in lipid biosynthesis and fatty acid synthesis. It is an interacting partner for phsophatidylcholine transfer protein which is a highly specific intracellular lipid-binding protein. ${ }^{\mathrm{S} 61}$.

The enzyme regulates the activity of ChREBP (carbohydrate response element-binding protein) which regulates expression of genes involved in hepatic glycolysis and lipogenesis ${ }^{\mathrm{S} 62}$.

Involved in gamma-glutamyl cycle which produces glutathione molecules and is important to protect cells from oxidative damage and plays a role in membrane transport of amino acids ${ }^{\mathrm{S} 63}$.

Predominately expressed in the liver and is the ratelimiting enzyme for glycogenesis ${ }^{\mathrm{S} 64}$.

Encodes hydroxyacid-oxoacid transhydrogenase which is responsible for the oxidation of 4-hydroxybutyrate to succinate semialdehyde. Studies have shown that Adhfe1 transcript dysregulation in obese adipose tissue and obese kidney influences obesity parameters ${ }^{\mathrm{S}}{ }^{\text {. }}$.

The gene is a microsomal enzyme that catalyzes the oxidation of medium- and long-chain aliphatic aldehydes to fatty acids ${ }^{\mathrm{S} 66}$. 
Supplementary Table 1 (Continued)

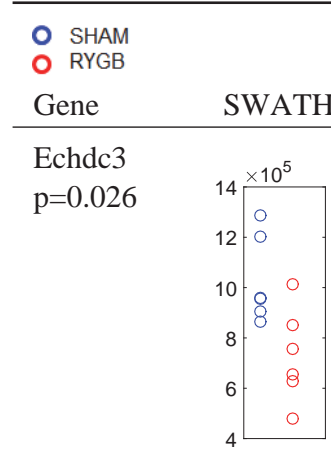

Acsf2 $\mathrm{p}=0.002$

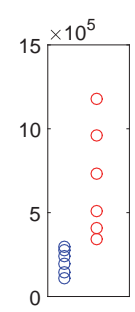

Acnat2

$\mathrm{p}=0.026$

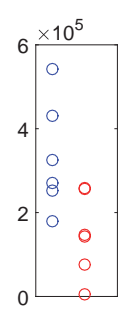

Hyi $\mathrm{p}=0.026$

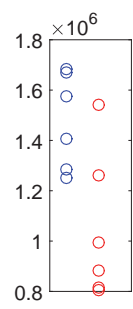

LOC103692171 $\mathrm{p}=0.015$

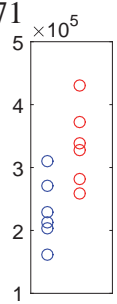

Scly $\mathrm{p}=0.041$

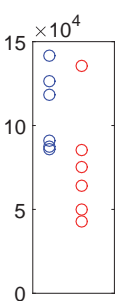

Name/Enzyme Commission \#

Enoyl coA hydratase

Fatty-acyl-coA synthetase

Acyl-coenzyme A amino acid Nacyltransferase 2

hydroxypyruvate isomerase

$\mathrm{n} / \mathrm{a}$

$\mathrm{n} / \mathrm{a}$

Selenocysteine lyase catalyzes the decomposition of selenocysteine into alanine and selenide. Mice lacking Scly have been shown to develop metabolic syndrome, hyperinsulinemia, hyperleptinemia, and hypercholesterolemia $^{\text {S71 }}$. 
Supplementary Table 1 (Continued)

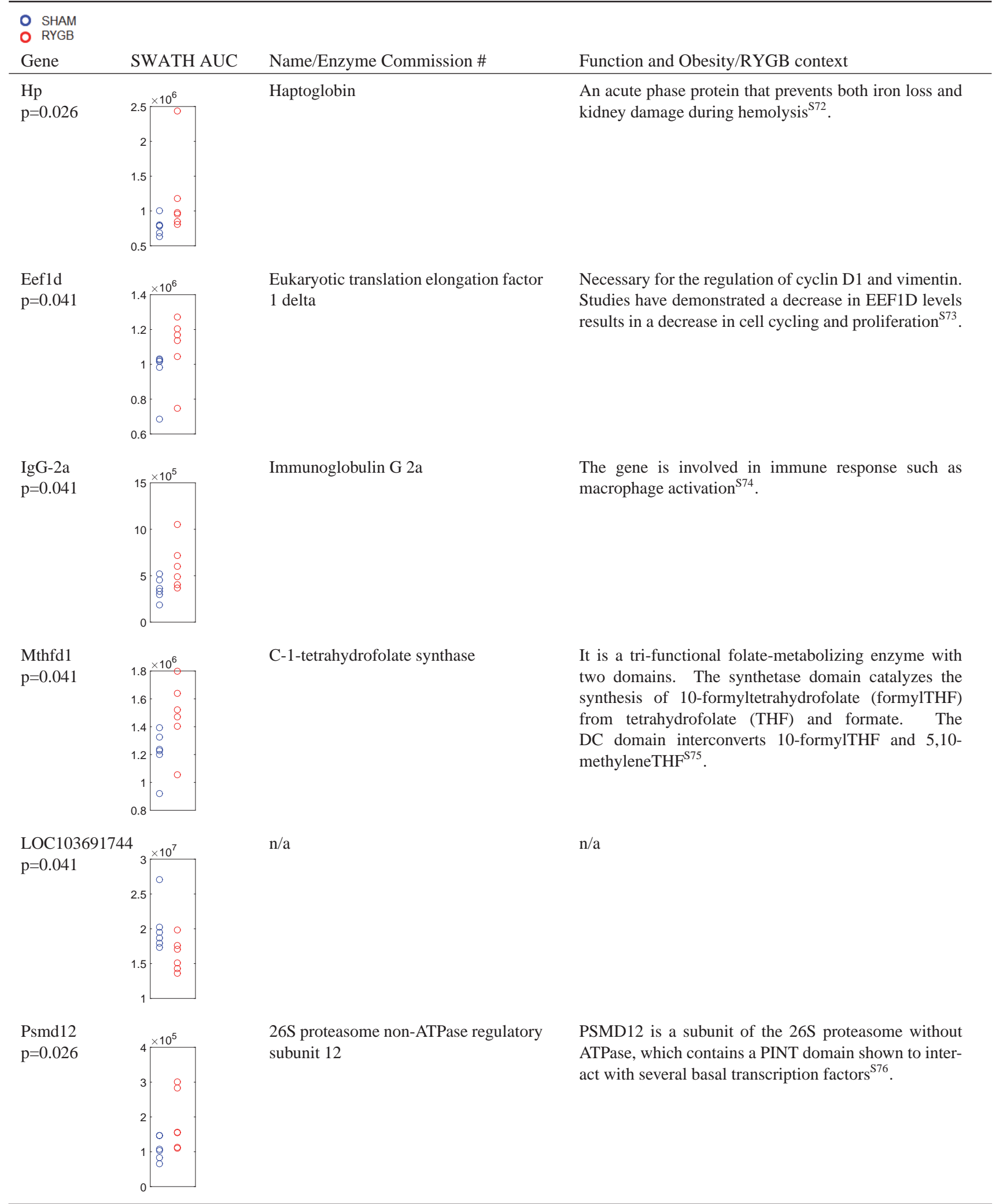


Supplementary Table 1 (Continued)

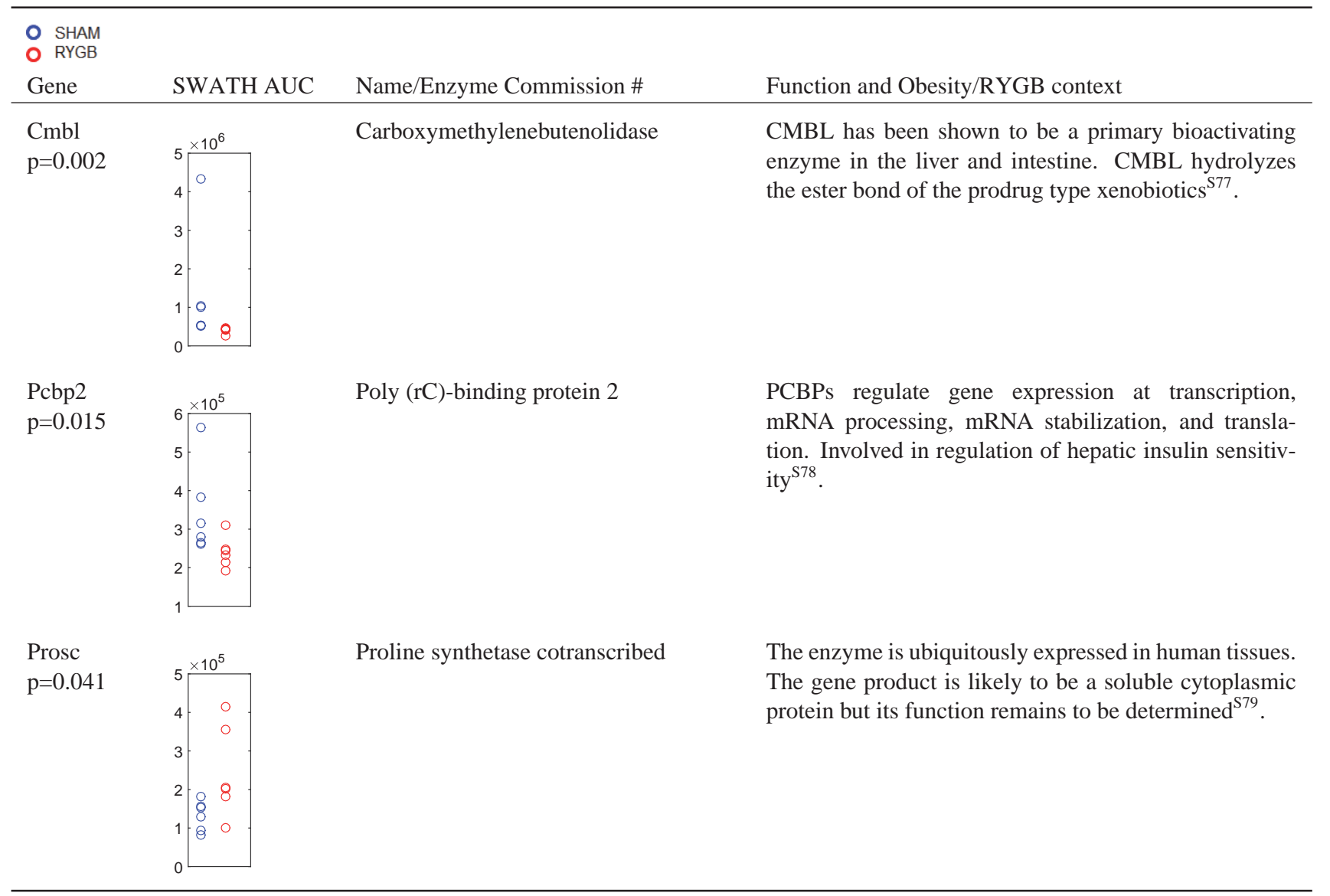


Supplementary Table $2{ }^{\mathrm{A}}$ Metabolic pathways or modules described in the KEGG Database; ${ }^{\mathrm{B}}$ Number of SSPs between Sham and RYGB KEGG pathway; ${ }^{\mathrm{C}}$ List of SSPs.

\begin{tabular}{|c|c|c|}
\hline KEGG Pathway ${ }^{\mathrm{A}}$ & \# of SSPs ${ }^{\mathrm{B}}$ & List of SSPs ${ }^{\mathrm{C}}$ \\
\hline Lysine degradation & 8 & Aass, Acat2, Pipox, Hykk, Aldh9a1, Hspa9, Aadat, Aldh3a2 \\
\hline Tryptophan metabolism & 6 & Acat2, Aldh9a1, Hspa9, Aadat, Cyp1a2, Aldh3a2 \\
\hline Peroxisome & 6 & Hao1, Ech1, Ephx2, Pipox, Idh2, Baat \\
\hline Biosynthesis of amino acids & 5 & Asl, Got1, Idh2, Aco2, Aadat \\
\hline Valine, leucine and isoleucine degradation & 5 & Acat2, Mut, Aldh9a1, Hspa9, Aldh3a2 \\
\hline Glyoxylate and dicarboxylate metabolism & 5 & Acat2, Hao1, Mut, Aco2, Hyi \\
\hline Histidine metabolism & 4 & Ftcd, Aldh9a1, Hspa9, Aldh3a2 \\
\hline Fatty acid degradation & 4 & Acat2, Aldh9a1, Hspa9, Aldh3a2 \\
\hline Pyruvate metabolism & 4 & Acat2, Aldh9a1, Hspa9, Aldh3a2 \\
\hline Arginine and proline metabolism & 4 & Got1, Aldh9a1, Hspa9, Aldh3a2 \\
\hline 2-Oxocarboxylic acid metabolism & 4 & Got1, Idh2, Aco2, Aadat \\
\hline Glycerolipid metabolism & 4 & Gk, Aldh9a1, Hspa9, Aldh3a2 \\
\hline PPAR signaling pathway & 3 & Fabp7, Slc27a5, Gk \\
\hline Cysteine and methionine metabolism & 3 & Got1, Bhmt, Gss \\
\hline Purine metabolism & 3 & Enpp3, Nme2, Adk \\
\hline Epstein-Barr virus infection & 3 & Vim, Ywhab, Psmd12 \\
\hline Glycolysis/Gluconeogenesis & 3 & Aldh9a1, Hspa9, Aldh3a2 \\
\hline Ascorbate and aldarate metabolism & 3 & Aldh9a1, Hspa9, Aldh3a2 \\
\hline beta-Alanine metabolism & 3 & Aldh9a1, Hspa9, Aldh3a2 \\
\hline Steroid biosynthesis & 2 & Fdft1, Ebp \\
\hline Arginine biosynthesis & 2 & Asl, Got1 \\
\hline Alanine, aspartate and glutamate metabolism & 2 & Asl, Got1 \\
\hline Lysosome & 2 & Glb1, Ctsc \\
\hline One carbon pool by folate & 2 & Ftcd, Mthfd 1 \\
\hline Propanoate metabolism & 2 & Acat2, Mut \\
\hline Pyrimidine metabolism & 2 & Tymp, Nme2 \\
\hline Primary bile acid biosynthesis & 2 & Slc27a5, Baat \\
\hline Insulin resistance & 2 & Slc27a5, Gys2 \\
\hline Bile secretion & 2 & Slc27a5, Baat \\
\hline Oxidative phosphorylation & 2 & Ndufs2, Ndufv2 \\
\hline Non-alcoholic fatty liver disease (NAFLD) & 2 & Ndufs2, Ndufv2 \\
\hline Alzheimer's disease & 2 & Ndufs2, Ndufv2 \\
\hline Parkinson's disease & 2 & Ndufs2, Ndufv2 \\
\hline Huntington's disease & 2 & Ndufs2, Ndufv2 \\
\hline Glycine, serine and threonine metabolism & 2 & Bhmt, Pipox \\
\hline Citrate cycle (TCA cycle) & 2 & Idh2, Aco2 \\
\hline Glutathione metabolism & 2 & Idh2, Gss \\
\hline Starch and sucrose metabolism & 2 & Enpp3, Gys2 \\
\hline Retinol metabolism & 2 & Сур2a2, Сур1а2 \\
\hline Systemic lupus erythematosus & 2 & Snrpd3, Actn 4 \\
\hline Drug metabolism - cytochrome P450 & 2 & Fmo1, Cyp1a2 \\
\hline Viral carcinogenesis & 2 & Actn4, Ywhab \\
\hline
\end{tabular}


Supplementary Table 3 Individual metabolite levels based on normalized peak areas comparing Sham and RYGB for SSMs.

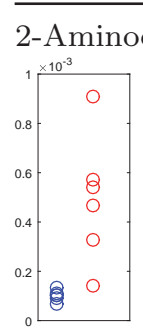

5-methoxytryptophan

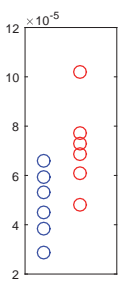

ADP-D-glucose

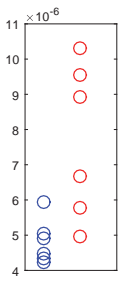

aspartate

\begin{tabular}{r|r|r|}
${ }_{4}^{4}$ & & 0 \\
3.5 & & 0 \\
3 & & 0 \\
3 & 0 & 0 \\
2.5 & 0 & 0 \\
2 & 0 & 0 \\
2 & 0 & \\
1.5 & 8 & \\
\hline & & \\
\hline
\end{tabular}

cellobiose

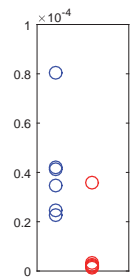

coenzyme A

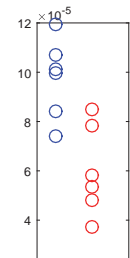

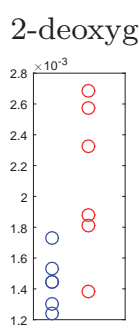

7-8-dihydrofolate

\begin{tabular}{r|rr|}
$r .5$ & 0 \\
$3.50^{-6}$ & & 0 \\
2.5 & & 0 \\
2 & 0 & \\
1.5 & 0 & 8 \\
1.5 & 0 \\
0.5 & & \\
0 & 0 & \\
\hline
\end{tabular}

ADP

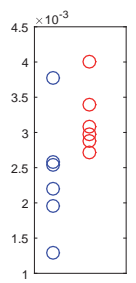

ATP-nega

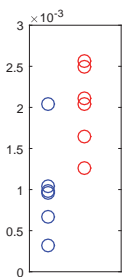

cholesteryl sulfate

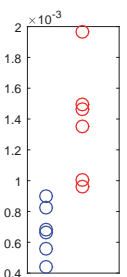

CTP

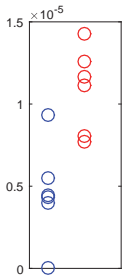

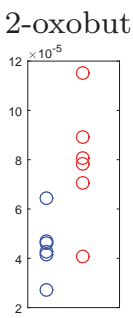

7-methylguanosine

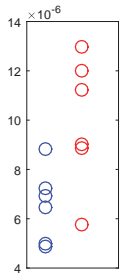

arginosuccinic-acid

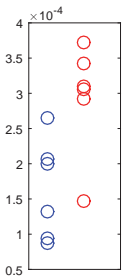

biotin

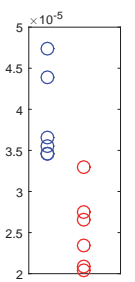

citrulline

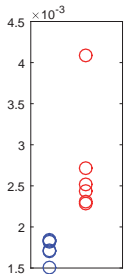

cyclic-AMP

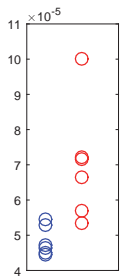

4-aminobutyrate

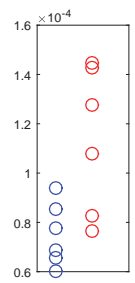

acetyl-CoA

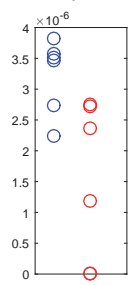

asparagine

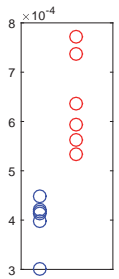

carbamoyl-phosphate

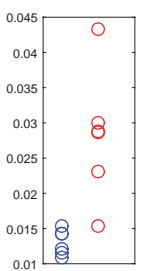

CMP

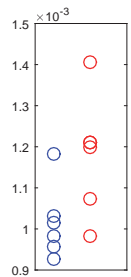

dATP

\begin{tabular}{|c|c|c|}
\hline $1.6 \times 10^{106}$ & 0 \\
1.4 & 0 \\
1.2 & 0 \\
1.2 & 0 \\
0.8 & \\
0.6 & \\
0.4 & & \\
0.2 & & \\
\hline 0 & 0 & 0 \\
\hline
\end{tabular} 
Supplementary Table 3 (Continued)

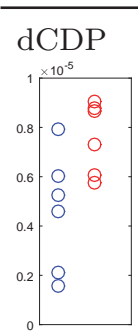

dGDP

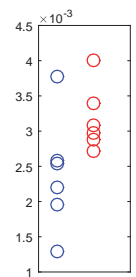

dihydroxy-acetone-phosphate

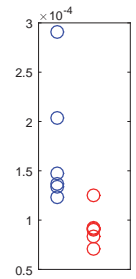

FAD

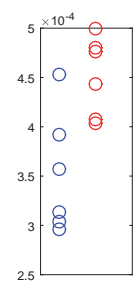

glutamate

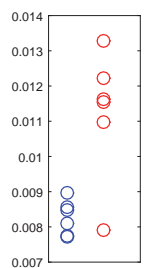

glycine

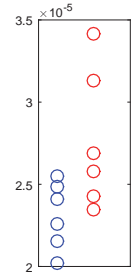

\section{deoxyguanosine}

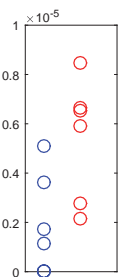

D-glucarate

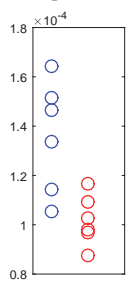

DL-Pipecolic acid

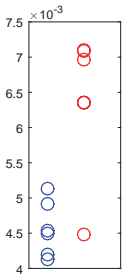

fructose-6-phosphate

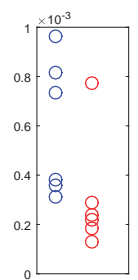

glutamine

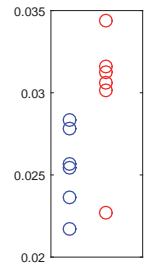

Guanidoacetic-acid

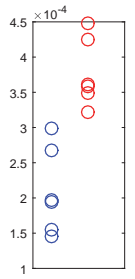

D-glyceraldehdye-3-phosphate

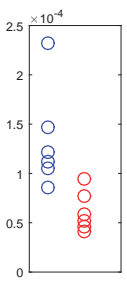

dTMP

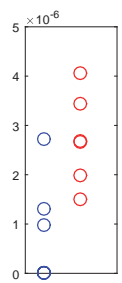

glucose-1-phosphate

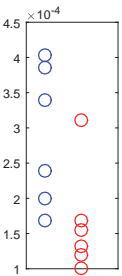

glutathione-disulfide

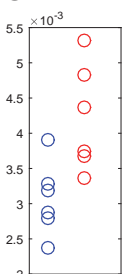

hexose-phosphate

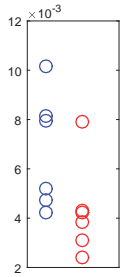

dephospho-CoA
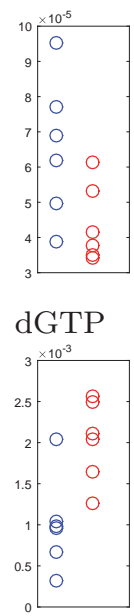

dUMP-nega

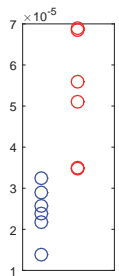

glucose-6-phosphate

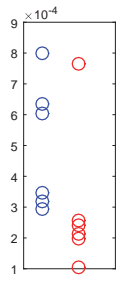

glutathione-

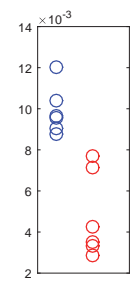

histidinol

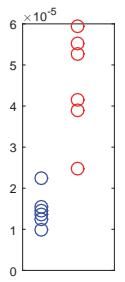

(Continued) 
Supplementary Table 3 (Continued)

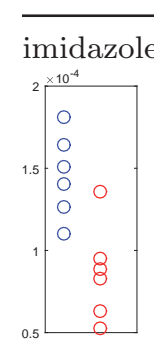

\section{NADPH}

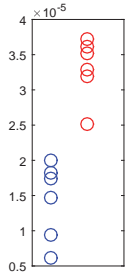

quinolinate

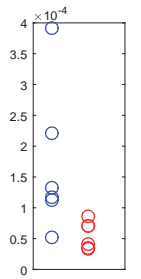

succinyl-CoA-posi

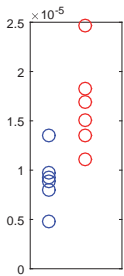

trans-trans-farnesyl-

diphosphate

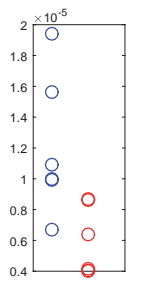

UDP

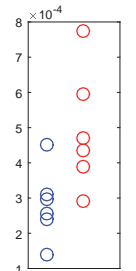

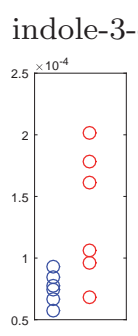

O8P-O1P

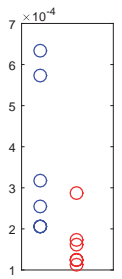

spermine

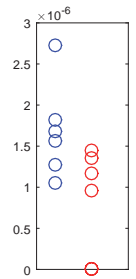

taurine

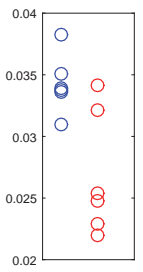

trehalose-sucrose

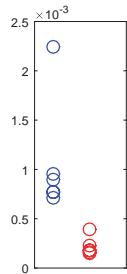

UTP

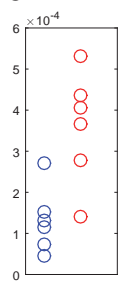

L-arginino-succinate

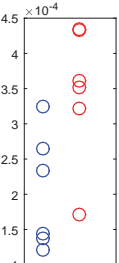

Pyroglutamic-acid

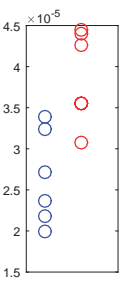

S-ribosyl-L-homocysteine-nega

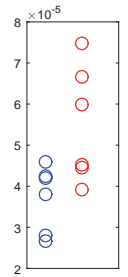

succinate

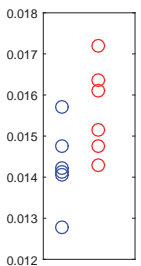

Thiamine-pyrophosphate

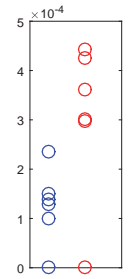

thymine

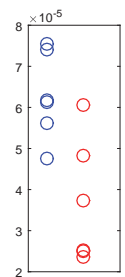

tyrosine

UDP-D-glucose
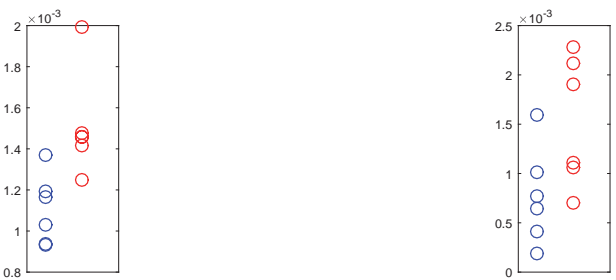
Supplementary Table $4{ }^{\mathrm{A}}$ Metabolic pathways or modules described in the KEGG Database; ${ }^{\mathrm{B}}$ Number of SSMs between Sham and RYGB KEGG pathway; ${ }^{\mathrm{C}}$ List of SSMs.

\begin{tabular}{|c|c|c|}
\hline KEGG Pathway ${ }^{\mathrm{A}}$ & $\begin{array}{l}\text { Num of } \\
\text { SSMs }^{\mathrm{B}}\end{array}$ & List of SSMs ${ }^{\mathrm{C}}$ \\
\hline Pyrimidine metabolism & 12 & $\begin{array}{l}\text { carbamoyl phosphate, dUMP, dCDP, UDP, dCTP, CTP, UTP, UDP-D-glucose, thymine, } \\
\text { glutamine, dTMP, guanidoacetic acid }\end{array}$ \\
\hline $\begin{array}{l}\text { Biosynthesis of alkaloids derived from ornithine, } \\
\text { lysine and nicotinic acid }\end{array}$ & 11 & $\begin{array}{l}\text { succinate, quinolinate, D-glyceraldehdye-3-phosphate, hexose-phosphate, glucose-6-phosphate, } \\
\text { fructose-6-phosphate, acetyl-CoA, DL-pipecolic acid, aspartate, glutamate, 2-keto-isovalerate }\end{array}$ \\
\hline Purine metabolism & 10 & $\begin{array}{l}\text { carbamoyl phosphate, cyclic-AMP, ADP, dGDP, dATP, ATP, dGTP, glycine, glutamine, maleic } \\
\text { acid }\end{array}$ \\
\hline Glutathione metabolism & 10 & $\begin{array}{l}\text { pyroglutamic acid, glutathione, NADPH, acetyl-CoA, glycine, glutamate, glutathione, } \\
\text { glutathione disulfide, NADPH, maleic acid }\end{array}$ \\
\hline $\mathrm{ABC}$ transporters & 10 & $\begin{array}{l}\text { taurine, glutathione, trehalose-sucrose, cellobiose, glycine, aspartate, glutamine, glutamate, } \\
\text { biotin, maleic acid }\end{array}$ \\
\hline Glycolysis/Gluconeogenesis & 9 & $\begin{array}{l}\text { lactate, dihydroxy-acetone-phosphate, D-glyceraldehdye-3-phosphate, hexose-phosphate, } \\
\text { glucose-1-phosphate, glucose-6-phosphate, fructose-6-phosphate, thiamine pyrophosphate, } \\
\text { fumarate }\end{array}$ \\
\hline Biosynthesis of plant hormones & 9 & $\begin{array}{l}\text { succinate, D-glyceraldehdye-3-phosphate, hexose-phosphate, glucose-6-phosphate, fructose-6- } \\
\text { phosphate, trans, trans-farnesyl diphosphate, acetyl-CoA, aspartate, fumarate }\end{array}$ \\
\hline Neuroactive ligand-receptor interaction & 9 & taurine, UDP, ADP, UTP, ATP, glycine, 4-aminobutyrate, aspartate, fumarate \\
\hline Central carbon metabolism in cancer & 9 & lactate, succinate, acetyl-CoA, glycine, asparagine, aspartate, glutamine, glutamate, fumarate \\
\hline Alanine, aspartate and glutamate metabolism & 8 & $\begin{array}{l}\text { succinate, carbamoyl phosphate, } 4 \text {-aminobutyrate, asparagine, aspartate, glutamine, glutamate, } \\
\text { uracil }\end{array}$ \\
\hline Starch and sucrose metabolism & 8 & $\begin{array}{l}\text { hexose-phosphate, glucose-1-phosphate, glucose-6-phosphate, fructose-6-phosphate, trehalose- } \\
\text { sucrose, cellobiose, UDP-D-glucose, uracil }\end{array}$ \\
\hline Biosynthesis of phenylpropanoids & 8 & $\begin{array}{l}\text { succinate, D-glyceraldehdye-3-phosphate, hexose-phosphate, glucose-6-phosphate, fructose-6- } \\
\text { phosphate, acetyl-CoA, 7,8-dihydrofolate, uracil }\end{array}$ \\
\hline $\begin{array}{l}\text { Biosynthesis of alkaloids derived from shikimate } \\
\text { pathway }\end{array}$ & 8 & $\begin{array}{l}\text { succinate, D-glyceraldehdye-3-phosphate, hexose-phosphate, glucose-6-phosphate, fructose-6- } \\
\text { phosphate, acetyl-CoA, tyrosine, uracil }\end{array}$ \\
\hline $\begin{array}{l}\text { Biosynthesis of alkaloids derived from terpenoid } \\
\text { and polyketide }\end{array}$ & 8 & $\begin{array}{l}\text { succinate, D-glyceraldehdye-3-phosphate, hexose-phosphate, glucose-6-phosphate, fructose-6- } \\
\text { phosphate, trans, trans-farnesyl diphosphate, acetyl-CoA, uracil }\end{array}$ \\
\hline $\begin{array}{l}\text { Biosynthesis of alkaloids derived from histidine } \\
\text { and purine }\end{array}$ & 7 & $\begin{array}{l}\text { succinate, D-glyceraldehdye-3-phosphate, hexose-phosphate, glucose-6-phosphate, fructose-6- } \\
\text { phosphate, acetyl-CoA, glycerate }\end{array}$ \\
\hline Galactose metabolism & 6 & $\begin{array}{l}\text { dihydroxy-acetone-phosphate, D-glyceraldehdye-3-phosphate, glucose-1-phosphate, glucose-6- } \\
\text { phosphate, trehalose-sucrose, acetoacetate }\end{array}$ \\
\hline Arginine biosynthesis & 6 & carbamoyl phosphate, aspartate, glutamine, glutamate, citrulline, acetoacetate \\
\hline Amino sugar and nucleotide sugar metabolism & 6 & $\begin{array}{l}\text { hexose-phosphate, glucose-1-phosphate, glucose-6-phosphate, fructose-6-phosphate, UDP-D- } \\
\text { glucose, acetoacetate }\end{array}$ \\
\hline Glyoxylate and dicarboxylate metabolism & 6 & succinate, acetyl-CoA, glycine, glutamine, glutamate, acetoacetate \\
\hline Propanoate metabolism & 6 & lactate, 2-oxobutanoate, succinate, dihydroxy-acetone-phosphate, acetyl-CoA, acetoacetate \\
\hline Aminoacyl-tRNA biosynthesis & 6 & glycine, asparagine, aspartate, glutamine, glutamate, acetoacetate \\
\hline Biosynthesis of terpenoids and steroids & 6 & $\begin{array}{l}\text { succinate, D-glyceraldehdye-3-phosphate, hexose-phosphate, fructose-6-phosphate, trans, trans- } \\
\text { farnesyl diphosphate, acetoacetate }\end{array}$ \\
\hline Thyroid hormone synthesis & 6 & glutathione, cyclic-AMP, NADPH, glutathione, glutathione disulfide, acetoacetate \\
\hline Glucagon signaling pathway & 6 & lactate, succinate, glucose-1-phosphate, glucose-6-phosphate, cyclic-AMP, acetoacetate \\
\hline Protein digestion and absorption & 6 & glycine, asparagine, aspartate, glutamine, glutamate, acetoacetate \\
\hline $\begin{array}{l}\text { Glycolysis (Embden-Meyerhof pathway) } \\
\text { glucose = pyruvate }\end{array}$ & 5 & $\begin{array}{l}\text { dihydroxy-acetone-phosphate, D-glyceraldehdye-3-phosphate, hexose-phosphate, glucose-6- } \\
\text { phosphate, 2-oxobutanoate }\end{array}$ \\
\hline $\begin{array}{l}\text { Pyrimidine deoxyribonuleotide biosynthesis, } \\
\text { CDP/CTP }=>\text { dCDP/dCTP,dTDP/dTTP }\end{array}$ & 5 & dUMP, dCDP, dCTP, CTP, 2-oxobutanoate \\
\hline Fructose and mannose metabolism & 5 & $\begin{array}{l}\text { lactate, dihydroxy-acetone-phosphate, D-glyceraldehdye-3-phosphate, hexose-phosphate, } \\
\text { 2-oxobutanoate }\end{array}$ \\
\hline Cysteine and methionine metabolism & 5 & 2-oxobutanoate, S-ribosyl-L-homocysteine-nega, glutathione, aspartate, 2-oxobutanoate \\
\hline Phenylalanine metabolism & 5 & succinate, phenylpyruvate, acetyl-CoA, tyrosine, 2-oxobutanoate \\
\hline beta-Alanine metabolism & 5 & quinolinate, acetyl-CoA, 4-aminobutyrate, aspartate, 2 -oxobutanoate \\
\hline
\end{tabular}


Supplementary Table 4 (Continued)

\begin{tabular}{|c|c|c|}
\hline KEGG Pathway ${ }^{\mathrm{A}}$ & $\begin{array}{l}\text { Num of } \\
\text { SSMs }^{\mathrm{B}}\end{array}$ & List of SSMs ${ }^{\mathrm{C}}$ \\
\hline Butanoate metabolism & 5 & succinate, acetyl-CoA, 4-aminobutyrate, glutamate, 2-oxobutanoate \\
\hline GABAergic synapse & 5 & succinate, cyclic-AMP, 4-aminobutyrate, glutamine, 2-oxobutanoate \\
\hline Bile secretion & 5 & glutathione, cyclic-AMP, acetyl-CoA, glutathione, 2-oxobutanoate \\
\hline Vitamin digestion and absorption & 5 & thiamine pyrophosphate, coenzyme A, biotin, coenzyme A-posi, 2-oxobutanoate \\
\hline Gluconeogenesis, oxaloacetate $=$ fructose $-6 \mathrm{P}$ & 4 & dihydroxy-acetone-phosphate, D-glyceraldehdye-3-phosphate, hexose-phosphate, lactate \\
\hline $\begin{array}{l}\text { Pentose phosphate pathway (Pentose phosphate } \\
\text { cycle) }\end{array}$ & 4 & D-glyceraldehdye-3-phosphate, hexose-phosphate, glucose-6-phosphate, lactate \\
\hline Urea cycle & 4 & carbamoyl phosphate, aspartate, citrulline, lactate \\
\hline Citrate cycle (TCA cycle) & 4 & succinate, thiamine pyrophosphate, acetyl-CoA, lactate \\
\hline Pentose phosphate pathway & 4 & D-glyceraldehdye-3-phosphate, hexose-phosphate, glucose-6-phosphate, lactate \\
\hline Glycine, serine and threonine metabolism & 4 & 2-oxobutanoate, guanidoacetic acid, glycine, lactate \\
\hline Arginine and proline metabolism & 4 & guanidoacetic acid, 4-aminobutyrate, glutamate, lactate \\
\hline Histidine metabolism & 4 & Imidazoleacetic acid, aspartate, histidinol, lactate \\
\hline Cyanoamino acid metabolism & 4 & glycine, asparagine, aspartate, lactate \\
\hline Pyruvate metabolism & 4 & lactate, succinate, thiamine pyrophosphate, lactate \\
\hline Methane metabolism & 4 & dihydroxy-acetone-phosphate, D-glyceraldehdye-3-phosphate, acetyl-CoA, lactate \\
\hline Thiamine metabolism & 4 & D-glyceraldehdye-3-phosphate, thiamine pyrophosphate, glycine, lactate \\
\hline Nicotinate and nicotinamide metabolism & 4 & quinolinate, dihydroxy-acetone-phosphate, 4 -aminobutyrate, lactate \\
\hline Pantothenate and CoA biosynthesis & 4 & dephospho-CoA-nega, coenzyme A, aspartate, lactate \\
\hline Two-component system & 4 & succinate, aspartate, glutamine, lactate \\
\hline cAMP signaling pathway & 4 & lactate, succinate, cyclic-AMP, lactate \\
\hline Synaptic vesicle cycle & 4 & ATP, glycine, 4-aminobutyrate, lactate \\
\hline Cholinergic synapse & 4 & cyclic-AMP, coenzyme A, acetyl-CoA, lactate \\
\hline Parkinson's disease & 4 & cyclic-AMP, ADP, ATP, lactate \\
\hline Citrate cycle (TCA cycle, Krebs cycle) & 3 & succinate, acetyl-CoA, pyruvate \\
\hline GABA (gamma-Aminobutyrate) shunt & 3 & succinate, 4 -aminobutyrate, pyruvate \\
\hline Reductive citrate cycle (Arnon-Buchanan cycle) & 3 & succinate, acetyl-CoA, pyruvate \\
\hline Dicarboxylate-hydroxybutyrate cycle & 3 & succinate, acetyl-CoA, pyruvate \\
\hline 3-Hydroxypropionate bi-cycle & 3 & succinate, acetyl-CoA, pyruvate \\
\hline Pentose and glucuronate interconversions & 3 & dihydroxy-acetone-phosphate, glucose-1-phosphate, pyruvate \\
\hline Fatty acid degradation & 3 & coenzyme A, acetyl-CoA, pyruvate \\
\hline Oxidative phosphorylation & 3 & succinate, $\mathrm{ADP}$, pyruvate \\
\hline Valine, leucine and isoleucine degradation & 3 & thiamine pyrophosphate, acetyl-CoA, pyruvate \\
\hline Lysine degradation & 3 & acetyl-CoA, glycine, pyruvate \\
\hline Carbapenem biosynthesis & 3 & coenzyme A, glutamate, pyruvate \\
\hline Taurine and hypotaurine metabolism & 3 & taurine, acetyl-CoA, pyruvate \\
\hline Inositol phosphate metabolism & 3 & dihydroxy-acetone-phosphate, D-glyceraldehdye-3-phosphate, pyruvate \\
\hline Terpenoid backbone biosynthesis & 3 & D-glyceraldehdye-3-phosphate, trans, trans-farnesyl diphosphate, pyruvate \\
\hline Nitrogen metabolism & 3 & carbamoyl phosphate, glutamine, pyruvate \\
\hline HIF-1 signaling pathway & 3 & lactate, ATP, pyruvate \\
\hline AMPK signaling pathway & 3 & glucose-6-phosphate, ADP, pyruvate \\
\hline Platelet activation & 3 & cyclic-AMP, ADP, pyruvate \\
\hline Retrograde endocannabinoid signaling & 3 & cyclic-AMP, 4-aminobutyrate, pyruvate \\
\hline Glutamatergic synapse & 3 & cyclic-AMP, glutamine, pyruvate \\
\hline Taste transduction & 3 & cyclic-AMP, trehalose-sucrose, pyruvate \\
\hline Renin secretion & 3 & cyclic-AMP, ADP, pyruvate \\
\hline
\end{tabular}


Supplementary Table 4 (Continued)

\begin{tabular}{|c|c|c|}
\hline KEGG Pathway ${ }^{\mathrm{A}}$ & $\begin{array}{l}\text { Num of } \\
\text { SSMs }^{B}\end{array}$ & List of SSMs ${ }^{\mathrm{C}}$ \\
\hline Mineral absorption & 3 & glycine, asparagine, pyruvate \\
\hline Cocaine addiction & 3 & cyclic-AMP, glutamate, pyruvate \\
\hline Amphetamine addiction & 3 & cyclic-AMP, glutamate, pyruvate \\
\hline Alcoholism & 3 & cyclic-AMP, glutamate, pyruvate \\
\hline Ethylmalonyl pathway & 2 & acetyl-CoA, glycolate \\
\hline Hydroxypropionate-hydroxybutylate cycle & 2 & acetyl-CoA, glycolate \\
\hline Photorespiration & 2 & glycine, glycolate \\
\hline Methylaspartate cycle & 2 & acetyl-CoA, glycolate \\
\hline Ascorbate and aldarate metabolism & 2 & D-glucarate, glycolate \\
\hline Primary bile acid biosynthesis & 2 & taurine, glycolate \\
\hline Puromycin biosynthesis & 2 & ATP, glycolate \\
\hline Monobactam biosynthesis & 2 & aspartate, glycolate \\
\hline Valine, leucine and isoleucine biosynthesis & 2 & 2-oxobutanoate, glycolate \\
\hline Lysine biosynthesis & 2 & acetyl-CoA, glycolate \\
\hline Tyrosine metabolism & 2 & succinate, glycolate \\
\hline Benzoate degradation & 2 & acetyl-CoA, glycolate \\
\hline Tryptophan metabolism & 2 & quinolinate, glycolate \\
\hline $\begin{array}{l}\text { Phenylalanine, tyrosine and tryptophan } \\
\text { biosynthesis }\end{array}$ & 2 & phenylpyruvate, glycolate \\
\hline D-Glutamine and D-glutamate metabolism & 2 & glutamine, glycolate \\
\hline Glycerolipid metabolism & 2 & dihydroxy-acetone-phosphate, glycolate \\
\hline C5-Branched dibasic acid metabolism & 2 & 2-oxobutanoate, glycolate \\
\hline Sulfur metabolism & 2 & succinate, glycolate \\
\hline $\begin{array}{l}\text { Tropane, piperidine and pyridine alkaloid } \\
\text { biosynthesis }\end{array}$ & 2 & phenylpyruvate, glycolate \\
\hline Phosphotransferase system (PTS) & 2 & trehalose-sucrose, glycolate \\
\hline Calcium signaling pathway & 2 & cyclic-AMP, glycolate \\
\hline FoxO signaling pathway & 2 & ADP, glycolate \\
\hline Lysosome & 2 & ADP, glycolate \\
\hline Gap junction & 2 & cyclic-AMP, glycolate \\
\hline Circadian entrainment & 2 & cyclic-AMP, glycolate \\
\hline Long-term potentiation & 2 & cyclic-AMP, glycolate \\
\hline Dopaminergic synapse & 2 & cyclic-AMP, glycolate \\
\hline $\begin{array}{l}\text { Inammatory mediator regulation of TRP } \\
\text { channels }\end{array}$ & 2 & cyclic-AMP, glycolate \\
\hline Insulin secretion & 2 & cyclic-AMP, glycolate \\
\hline Estrogen signaling pathway & 2 & cyclic-AMP, glycolate \\
\hline Melanogenesis & 2 & cyclic-AMP, glycolate \\
\hline Prolactin signaling pathway & 2 & ATP, glycolate \\
\hline Proximal tubule bicarbonate reclamation & 2 & glutamine, glycolate \\
\hline Fat digestion and absorption & 2 & coenzyme A, glycolate \\
\hline Morphine addiction & 2 & cyclic-AMP, glycolate \\
\hline Nicotine addiction & 2 & 4-aminobutyrate, glycolate \\
\hline Pertussis & 2 & cyclic-AMP, glycolate \\
\hline
\end{tabular}


Supplementary Table $5{ }^{\mathrm{A}}$ Metabolic pathways described in the KEGG Database; ${ }^{\mathrm{B}}$ Number of genes in the rat genome that are catalogued as being part of the KEGG pathway; ${ }^{C}$ Number of proteins detected in rat livers (at least one sample) using SWATH; ${ }^{\mathrm{D}}$ Fraction of all rat genes in the KEGG pathway detected in rat liver using SWATH proteomics; ${ }^{\mathrm{E}}$ List of proteins detected from the KEGG pathway in at least one sample.

\begin{tabular}{|c|c|c|c|c|}
\hline KEGG Pathway $^{\mathrm{A}}$ & Rat Genes ${ }^{\text {B }}$ & SWATH Proteins ${ }^{\mathrm{C}}$ & Coverage $^{\mathrm{D}}$ & List of Proteins Detected ${ }^{\mathrm{E}}$ \\
\hline $\begin{array}{l}\text { Glyoxylate and dicarboxylate } \\
\text { metabolism }\end{array}$ & 28 & 28 & 1.000 & $\begin{array}{l}\text { Acat2, Hao1, Mut, Aco2, Hyi, Hao2, Mdh1, Shmt1, Agxt, Afmid, } \\
\text { Mcee, Gldc, Pcca, Shmt2, Gcsh, Mdh2, Hoga1, Pccb, Grhpr, Acat1, } \\
\text { Gldc, Dld, Pccb, Glyctk, Cs, Aco1, Amt, Glul }\end{array}$ \\
\hline Lysine biosynthesis & 2 & 2 & 1.000 & Aadat, Aldh7a1 \\
\hline Citrate cycle (TCA cycle) & 32 & 26 & 0.813 & $\begin{array}{l}\text { Idh2, Aco2, Mdh1, Pck2, Sdhd, Pdhb, Pck1, Idh3a, Mdh2, Pdha1, } \\
\text { Idh3B, Sucla2, Suclg1, Dld, Dlat, Dlst, Sdha, Acly, Suclg2, Cs, } \\
\text { Idh3g, Aco1, Idh1, Sdhc, Ogdh, Sdhb }\end{array}$ \\
\hline Propanoate metabolism & 32 & 25 & 0.781 & $\begin{array}{l}\text { Ldha, Acat2, Mut, Acss3, Mlycd, Ehhadh, Abat, Mcee, Acaca, Dbt, } \\
\text { Hibch, Pcca, Bckdha, Aldh6a1, Acadm, Pccb, Sucla2, Acat1, } \\
\text { Suclg1, Dld, Pccb, Hadha, Bckdhb, Suclg2, Echs1 }\end{array}$ \\
\hline $\begin{array}{l}\text { Valine, leucine and isoleucine } \\
\text { degradation }\end{array}$ & 56 & 42 & 0.750 & $\begin{array}{l}\text { Acat2, Mut, Aldh9a1, Hspa9, Hsd17b10, Aldh3a2, Acaa2, Hmgcs1, } \\
\text { Aldh1b1, Ehhadh, Abat, Acad8, Mpv17, Mcee, Auh, Dbt, Acads, } \\
\text { Hibch, Pcca, Hmgcs2, Aox1, Bckdha, Agxt2, Acaa1a, Aldh6a1, } \\
\text { Acadm, Pccb, Acadsb, Mccc1, Aldh7a1, Acat1, Aox3, Dld, Pccb, } \\
\text { Hadha, Hibadh, Mccc2, Bckdhb, Hmgcl, Ivd, Echs1, Hadhb }\end{array}$ \\
\hline Fatty acid degradation & 47 & 33 & 0.702 & $\begin{array}{l}\text { Acat2, Aldh9a1, Hspa9, Aldh3a2, Acaa2, Aldh1b1, Adh6, Ehhadh, } \\
\text { Acox1, Acads, Cyp4a14, Acaa1a, Acsl1, Acadm, Acadsb, Aldh7a1, } \\
\text { Acat1, Acadl, Acs14, Eci2, Cpt1a, Hadha, Acox3, Cyp4a2, Fahd2, } \\
\text { Acs15, Echs1, Adh5, Cpt2, Eci1, Hadhb, Gcdh, Acadvl }\end{array}$ \\
\hline $\begin{array}{l}\text { D-Glutamine and D-glutamate } \\
\text { metabolism }\end{array}$ & 3 & 2 & 0.667 & Gls2, Glud1 \\
\hline Proteasome & 47 & 31 & 0.660 & $\begin{array}{l}\text { Psmd7, Psmb10, Psmd13, Psmd14, Psmc2, Psme1, Hadh, Psmd12, } \\
\text { Psma2, Psmd1, Psma6, Psma1, Psmc1, Psmd11, Psmd3, Psma4, } \\
\text { Psmc5, Psmb3, Psmd8, Psme2, Psmc6, Psmc4, Psma5, Psmb4, } \\
\text { Psma7, Atp6v1a, Psmc3, Psmb2, Psmd2, Psmb1, Psmb8 }\end{array}$ \\
\hline 2-Oxocarboxylic acid metabolism & 19 & 12 & 0.632 & $\begin{array}{l}\text { Got1, Idh2, Aco2, Aadat, Acy1a, Idh3a, Idh3B, Cs, Idh3g, Aco1, } \\
\text { Got2, Idh1 }\end{array}$ \\
\hline Primary bile acid biosynthesis & 16 & 10 & 0.625 & $\begin{array}{l}\text { Acox2, Slc27a5, Akr1d1, Cyp27a1, Baat, Akr1d1, Amacr, Hsd17b4, } \\
\text { Cyp8b1, Scp2 }\end{array}$ \\
\hline $\begin{array}{l}\text { Glycine, serine and threonine } \\
\text { metabolism }\end{array}$ & 40 & 24 & 0.600 & $\begin{array}{l}\text { Bhmt, Pipox, Gamt, Shmt1, Gcat, Agxt, Cbs, Sds, Gldc, Shmt2, Gcsh, } \\
\text { Agxt2, Aldh7a1, Grhpr, Sardh, Gldc, Dld, Glyctk, Chdh, Maob, } \\
\text { Amt, Pgam1, Maoa, Dmgdh }\end{array}$ \\
\hline Vitamin B6 metabolism & 10 & 6 & 0.600 & RGD1566085, Pdxk, Aox1, Pnpo, Aox3, Rbp1 \\
\hline Carbon metabolism & 121 & 72 & 0.595 & $\begin{array}{l}\text { Acat2, Hao1, Got1, Cps1, Idh2, Mut, Esd, Aco2, Hao2, Aldoa, Mdh1, } \\
\text { Idnk, Ighm, Shmt1, Aldob, Pgls, Sdhd, Agxt, Ehhadh, Sds, Pdhb, } \\
\text { Mcee, Gldc, Idh3a, Acads, Pgd, Impa1, Hibch, Pcca, Shmt2, Mdh2, } \\
\text { Pdha1, Idh3B, Fbp1, Aldh6a1, Acadm, Pccb, Sucla2, Gapdh, } \\
\text { Acat1, Suclg1, Pfkl, Gldc, Eno1, Pklr, Dld, Dlat, Pccb, Rgn, Dlst, } \\
\text { Hadha, Sdha, Taldo1, Suclg2, Cs, Glud1, Idh3g, Aco1, Got2, Tpi1, } \\
\text { Me1, Idh1, Tkt, Pgk1, Amt, Echs1, Pgam1, Sdhc, Adh5, Ogdh, } \\
\text { Tkfc, Sdhb }\end{array}$ \\
\hline Butanoate metabolism & 28 & 16 & 0.571 & $\begin{array}{l}\text { Acat2, Acsm3, Hmgcs1, Ehhadh, Abat, Bdh1, Acads, Hmgcs2, } \\
\text { L2hgdh, Acat1, Aldh5a1, Hadha, Acsm1, Hmgcl, Echs1, Acsm5 }\end{array}$ \\
\hline Tryptophan metabolism & 46 & 26 & 0.565 & $\begin{array}{l}\text { Acat2, Aldh9a1, Hspa9, Aadat, Cyp1a2, Aldh3a2, Acmsd, Cyp2c11, } \\
\text { Aldh1b1, Haao, Ehhadh, Afmid, Ccbl2, Ccbl1, Aox1, Aldh7a1, } \\
\text { Acat1, Aox3, Hadha, Kmo, Maob, Echs1, Kynu, Maoa, Ogdh, } \\
\text { Gcdh }\end{array}$ \\
\hline Arginine biosynthesis & 20 & 11 & 0.550 & Asl, Got1, Cps1, Arg1, Ass1, Gls2, Acy1a, Glud1, Otc, Got2, Glul \\
\hline Pyruvate metabolism & 40 & 22 & 0.550 & $\begin{array}{l}\text { Ldha, Acat2, Aldh9a1, Hspa9, Aldh3a2, Mdh1, Pck2, Aldh1b1, Pdhb, } \\
\text { Pck1, Acaca, Mdh2, Pdha1, Aldh7a1, Grhpr, Acat1, Pklr, Dld, } \\
\text { Dlat, Hagh, Me1, Glo1 }\end{array}$ \\
\hline $\begin{array}{l}\text { Synthesis and degradation of ketone } \\
\text { bodies }\end{array}$ & 11 & 6 & 0.545 & Acat2, Hmgcs1, Bdh1, Hmgcs2, Acat1, Hmgcl \\
\hline $\begin{array}{l}\text { Proximal tubule bicarbonate } \\
\text { reclamation }\end{array}$ & 22 & 12 & 0.545 & $\begin{array}{l}\text { Atp1b1, Mdh1, Pck2, Atp1b3, Nat1, Gls2, Pck1, Atp1a1, Slc25a10, } \\
\text { Adh1, Ca2, Glud1 }\end{array}$ \\
\hline
\end{tabular}


Supplementary Table 5 (Continued)

\begin{tabular}{|c|c|c|c|c|}
\hline KEGG Pathway ${ }^{\mathrm{A}}$ & Rat Genes ${ }^{\mathrm{B}}$ & SWATH Proteins ${ }^{\mathrm{C}}$ & Coverage $^{\mathrm{D}}$ & List of Proteins Detected ${ }^{\mathrm{E}}$ \\
\hline Histidine metabolism & 25 & 13 & 0.520 & $\begin{array}{l}\text { Ftcd, Aldh9a1, Hspa9, Aldh3a2, Cndp2, Aldh1b1, Uroc1, Amdhd1, } \\
\text { Vars, Aldh7a1, Hal, Maob, Maoa }\end{array}$ \\
\hline Ascorbate and aldarate metabolism & 27 & 14 & 0.519 & $\begin{array}{l}\text { Aldh9a1, Hspa9, Aldh3a2, Ugt2b17, Gulo, Ugt1a1, Ugt2b37, } \\
\text { Aldh1b1, Ugt2b15, Ugt2b10, Ugdh, Aldh7a1, Rgn, Ugt2b17 }\end{array}$ \\
\hline Fatty acid metabolism & 54 & 27 & 0.500 & $\begin{array}{l}\text { Acat2, Acaa2, Pecr, Ehhadh, Acaca, Acox1, Fasn, Acads, Acaa1a, } \\
\text { Acsl1, Acadm, Acadsb, Mecr, Acat1, Hsd17b12, Acadl, Acsl4, } \\
\text { Cpt1a, Hadha, Oxsm, Acox3, Tecr, Acs15, Echs1, Cpt2, Hadhb, } \\
\text { Acadvl }\end{array}$ \\
\hline Glutathione metabolism & 58 & 29 & 0.500 & $\begin{array}{l}\text { Idh2, Gss, Gstm3, Gstk1, Ndufv3, Gpx3, Lap3, Gsr, Mgst1, Gstp1, } \\
\text { Pgd, Impa1, Gstt1, Mgst3, Anpep, Gstm4, Gsto1, Gstt2, Gclc, } \\
\text { Txndc12, Gclm, Gsta1, Gstm1, Gsta4, Oplah, Gpx1, Idh1, Gsta1, } \\
\text { Gstm2 }\end{array}$ \\
\hline beta-Alanine metabolism & 34 & 17 & 0.500 & $\begin{array}{l}\text { Aldh9a1, Hspa9, Aldh3a2, Cndp2, Aldh1b1, Mlycd, Ehhadh, Abat, } \\
\text { Dpyd, Upb1, Dpys, Hi-bch, Aldh6a1, Acadm, Aldh7a1, Hadha, } \\
\text { Echs1 }\end{array}$ \\
\hline Caffeine metabolism & 6 & 3 & 0.500 & Cyp1a2, Uox, Xdh \\
\hline $\begin{array}{l}\text { Pentose and glucuronate } \\
\text { interconversions }\end{array}$ & 36 & 17 & 0.472 & $\begin{array}{l}\text { Aldh3a2, Ugt2b17, Ugt1a1, Akr1b1, Ugt2b37, Gusb, Aldh1b1, } \\
\text { Cryl1, Xylb, Sord, Ugt2b15, Ugt2b10, Ugdh, Ugp2, Akr1a1, Dcxr, } \\
\text { Ugt2b17 }\end{array}$ \\
\hline Sulfur metabolism & 13 & 6 & 0.462 & Suox, Gpt, Tst, Mpst, Ethe1, Papss2 \\
\hline Drug metabolism - cytochrome P450 & 71 & 32 & 0.451 & $\begin{array}{l}\text { Fmo1, Cyp1a2, Ugt2b17, Ugt1a1, Gstm3, Gstk1, Ndufv3, Ugt2b37, } \\
\text { Fmo3, Adh6, Mgst1, Fmo5, Gstp1, Ugt2b15, Ugt2b10, Aox1, } \\
\text { Gstt1, Mgst3, Gstm4, Aox3, Gsto1, Gstt2, Cyp2e1, Gsta1, Gstm1, } \\
\text { Gsta4, Maob, Gsta1, Gstm2, Adh5, Maoa, Ugt2b17 }\end{array}$ \\
\hline Pentose phosphate pathway & 29 & 13 & 0.448 & $\begin{array}{l}\text { Aldoa, Rbks, Idnk, Ighm, Aldob, Pgls, Pgd, Impa1, Fbp1, Pfkl, Rgn, } \\
\text { Taldo1, Tkt }\end{array}$ \\
\hline One carbon pool by folate & 18 & 8 & 0.444 & Ftcd, Shmt1, Mthfd1, Shmt2, Aldh1l1, Aldh112, Atic, Amt \\
\hline Nitrogen metabolism & 18 & 8 & 0.444 & Cps1, Ca3, Ca1, Car14, Ca5a, Ca2, Glud1, Glul \\
\hline Peroxisome & 88 & 39 & 0.443 & $\begin{array}{l}\text { Acox2, Hao1, Ech1, Ephx2, Pipox, Dhrs4, Idh2, Baat, Hao2, Gstk1, } \\
\text { Ndufv3, Pxmp2, Pecr, Mlycd, Phyh, Agxt, Ehhadh, Hacl1, Acox1, } \\
\text { Slc27a2, Sars, Amacr, Acaala, Abcd3, Acsl1, Sod2, Hsd17b4, } \\
\text { Acsl4, Eci2, Sod1, Acox3, Hmgcl, Scp2, Decr2, Idh1, Xdh, Acsl5, } \\
\text { Crot, Prdx5 }\end{array}$ \\
\hline Biosynthesis of amino acids & 83 & 36 & 0.434 & $\begin{array}{l}\text { Asl, Got1, Cps1, Idh2, Aco2, Aadat, Arg1, Aldoa, Shmt1, Aldob, Cbs, } \\
\text { Ass1, Sds, Acy1a, Idh3a, Shmt2, Idh3B, Aldh7a1, Gapdh, Pfkl, } \\
\text { Eno1, Pklr, Pah, Taldo1, Cs, Otc, Idh3g, Aco1, Got2, Tpi1, Idh1, } \\
\text { Tkt, Pgk1, Pgam1, Mat1a, Glul }\end{array}$ \\
\hline $\begin{array}{l}\text { Phenylalanine, tyrosine and } \\
\text { tryptophan biosynthesis }\end{array}$ & 7 & 3 & 0.429 & Got1, Pah, Got2 \\
\hline $\begin{array}{l}\text { Metabolism of xenobiotics by } \\
\text { cytochrome P450 }\end{array}$ & 70 & 30 & 0.429 & $\begin{array}{l}\text { Cyp1a2, Ugt2b17, Ugt1a1, Gstm3, Ephx1, Gstk1, Ndufv3, Ugt2b37, } \\
\text { Adh6, Mgst1, Gstp1, Ugt2b15, Ugt2b10, Gstt1, Mgst3, Gstm4, } \\
\text { Gsto1, Gstt2, Cyp2e1, Gsta1, Gstm1, Hsd11b1, Gsta4, Akr7a2, } \\
\text { Gsta1, Gstm2, Sult2a1, Adh5, Ugt2b17, Akr7a3 }\end{array}$ \\
\hline Fatty acid biosynthesis & 14 & 6 & 0.429 & Acaca, Fasn, Acsl1, Acsl4, Oxsm, Acsl5 \\
\hline Cysteine and methionine metabolism & 47 & 20 & 0.426 & $\begin{array}{l}\text { Ldha, Got1, Bhmt, Gss, Mdh1, Cbs, Mri1, Ahcy, Sds, Bhmt2, Adi1, } \\
\text { Mdh2, Agxt2, Tst, Mpst, Mtap, Gclc, Gclm, Got2, Mat1a }\end{array}$ \\
\hline Selenocompound metabolism & 19 & 8 & 0.421 & Txnrd2, Scly, Ccbl2, Ccbl1, Sephs1, Txnrd1, Mars, Papss2 \\
\hline PPAR signaling pathway & 79 & 33 & 0.418 & $\begin{array}{l}\text { Acox2, Fabp7, Slc27a5, Gk, Cyp27a1, Apoc3, Pck2, Fabp1, Ehhadh, } \\
\text { Pck1, Acox1, Slc27a2, Sars, Hmgcs2, Cyp4a14, Acaa1a, Acsl1, } \\
\text { Acadm, Acadl, Acs14, Apoa1, Cpt1a, Acox3, Cyp8b1, Cyp4a2, } \\
\text { Fahd2, Scp2, Dbi, Ilk, Me1, Acsl5, Cpt2, Apoa2 }\end{array}$ \\
\hline Chemical carcinogenesis & 91 & 38 & 0.418 & $\begin{array}{l}\text { Cyp1a2, Cyp3a18, Ugt2b17, Ugt1a1, Gstm3, Ephx1, Gstk1, Ndufv3, } \\
\text { Ugt2b37, Adh6, Mgst1, Cyp2c22, Ccbl2, Ccbl1, Gstp1, Ugt2b15, } \\
\text { Ugt2b10, Cyp3a2, Gstt1, Mgst3, Gstm4, Sult1a1, Cyp2c23, } \\
\text { Cyp2c7, Cyp2c13, Gsto1, Gstt2, Cyp2e1, Gsta1, Gstm1, Hsd11b1, } \\
\text { Gsta4, Cyp2b3, Gsta1, Gstm2, Sult2a1, Adh5, Ugt2b17 }\end{array}$ \\
\hline Phenylalanine metabolism & 22 & 9 & 0.409 & Got1, Hpd, Pah, Glyatl1, Got2, Glyat, Maob, Mif, Maoa \\
\hline
\end{tabular}


Supplementary Table 5 (Continued)

\begin{tabular}{|c|c|c|c|c|}
\hline KEGG Pathway ${ }^{\mathrm{A}}$ & Rat Genes ${ }^{\mathrm{B}}$ & SWATH Proteins ${ }^{\mathrm{C}}$ & Coverage $^{\mathrm{D}}$ & List of Proteins Detected ${ }^{\mathrm{E}}$ \\
\hline Oxidative phosphorylation & 144 & 58 & 0.403 & $\begin{array}{l}\text { Ndufs2, Ndufv2, Ndufa6, Uqcrc2, Ndufc2, Atp6v1e1, Ndufs1, } \\
\text { Cox7a2, Cox4i1, Ndufb4, Ndufb5, Atp6v1f, Uqcr10, Ndufb10, } \\
\text { Cox5b, Sdhd, Ndufs8, Uqcrq, Ndufs7, Atp5f1, Ndufa8, Ndufa12, } \\
\text { Ndufa5, Atp5j2, Atp6v0d1, Ndufa4, Atp5o, Ndufv1, Uqcrc1, } \\
\text { Ndufa9, Atp5b, Ndufab1, Ndufs4, Atp5j, Ndufs3, Ndufa2, Atp5d, } \\
\text { Atp5h, Ndufa10, Ndufa7, Ndufb7, Atp5i, Cox6c2, Uqcr11, } \\
\text { Cox6a1, Sdha, Cox6b1, Atp5a1, Ndufb3, Lhpp, Atp51, Sdhc, Ppa1, } \\
\text { Cyc1, Uqcrfs1, Ppa2, Cox5a, Sdhb }\end{array}$ \\
\hline $\begin{array}{l}\text { Alanine, aspartate and glutamate } \\
\text { metabolism }\end{array}$ & 35 & 14 & 0.400 & $\begin{array}{l}\text { Asl, Got1, Cps1, Adsl, Agxt, Ass1, Abat, Gls2, Nit2, Agxt2, Aldh5a1, } \\
\text { Glud1, Got2, Glul }\end{array}$ \\
\hline Glycolysis/Gluconeogenesis & 70 & 27 & 0.386 & $\begin{array}{l}\text { Ldha, Aldh9a1, Hspa9, Aldh3a2, Aldoa, Pck2, Aldh1b1, Aldob, } \\
\text { Adh6, Pdhb, Pck1, Pdha1, Fbp1, Aldh7a1, Gapdh, Pfkl, Galm, } \\
\text { Eno1, Pklr, Dld, Dlat, G6pc, Akr1a1, Tpi1, Pgk1, Pgam1, Adh5 }\end{array}$ \\
\hline Tyrosine metabolism & 39 & 15 & 0.385 & $\begin{array}{l}\text { Got1, Hpd, Adh6, Fah, Hgd, Aox1, Aox3, Gstz1, Fahd1, Got2, Maob, } \\
\text { Mif, Adh5, Maoa, Comt }\end{array}$ \\
\hline Parkinson's disease & 153 & 58 & 0.379 & $\begin{array}{l}\text { Ndufs2, Ndufv2, Ndufa6, Uqcrc2, Ndufc2, Ndufs1, Cox7a2, Cox4i1, } \\
\text { Ndufb4, Ndufb5, Uqcr10, Ndufb10, Cox5b, Sdhd, Ndufs8, Uqcrq, } \\
\text { Ndufs7, Atp5f1, Ndufa8, Ndufa12, Vdac2, Ndufa5, Uba1, Htra2, } \\
\text { Ndufa4, Prkaca, Atp5o, Ndufv1, Slc25a5, Uqcrc1, Ndufa9, Atp5b, } \\
\text { Ndufab1, Ndufs4, Atp5j, Ndufs3, Ndufa2, Atp5d, Atp5h, Ndufa10, } \\
\text { Ndufa7, Ndufb7, Cox6c2, Uqcr11, Cox6a1, Sdha, Vdac3, Ppif, } \\
\text { Cox6b1, Atp5a1, Ndufb3, Vdac1, Park7, Sdhc, Cyc1, Uqcrfs1, } \\
\text { Cox5a, Sdhb }\end{array}$ \\
\hline Lysine degradation & 52 & 19 & 0.365 & $\begin{array}{l}\text { Aass, Acat2, Pipox, Hykk, Aldh9a1, Hspa9, Aadat, Aldh3a2, } \\
\text { Aldh1b1, Ehhadh, Phykpl, Aldh7a1, Acat1, Dlst, Hadha, Bbox1, } \\
\text { Echs1, Ogdh, Gcdh }\end{array}$ \\
\hline Retinol metabolism & 83 & 30 & 0.361 & $\begin{array}{l}\text { Dhrs4, Cyp2a2, Cyp1a2, Cyp3a18, Ugt2b17, Ugt1a1, Ugt2b37, } \\
\text { Aldh1a1, Adh6, Hsd17b6, Cyp2c22, Cyp2a1, Cyp4a14, Ugt2b15, } \\
\text { Ugt2b10, Cyp3a2, Aox1, Cyp2c23, Cyp2c7, Aldh1a7, Aox3, } \\
\text { Cyp2c13, Aldh2, Cyp4a2, Fahd2, Cyp2b3, Retsat, Adh5, Ugt2b17, } \\
\text { Rdh3 }\end{array}$ \\
\hline Drug metabolism - other enzymes & 56 & 20 & 0.357 & $\begin{array}{l}\text { Tymp, Ces1e, Ugt2b17, Ugt1a1, Ces2a, Ugt2b37, Gusb, Hprt1, Dpyd, } \\
\text { Upb1, Dpys, Itpa, Ugt2b15, Ugt2b10, Tpmt, Umps, Xdh, Ces1d, } \\
\text { Alb, Ugt2b17 }\end{array}$ \\
\hline Arginine and proline metabolism & 51 & 18 & 0.353 & $\begin{array}{l}\text { Got1, Gamt, Aldh9a1, Hspa9, Aldh3a2, Agmat, Arg1, Cndp2, } \\
\text { Aldh1b1, Prodh, Lap3, Prodh2, Hoga1, Aldh7a1, Got2, Maob, } \\
\text { Oat, Maoa }\end{array}$ \\
\hline $\begin{array}{l}\text { Biosynthesis of unsaturated fatty } \\
\text { acids }\end{array}$ & 29 & 10 & 0.345 & $\begin{array}{l}\text { Baat, Acot2, Pecr, Acot4, Acox1, Acaala, Hsd17b12, Hadha, Acox3, } \\
\text { Tecr }\end{array}$ \\
\hline Steroid hormone biosynthesis & 81 & 27 & 0.333 & $\begin{array}{l}\text { Akr1d1, Cyp1a2, Cyp3a18, Ugt2b17, Ugt1a1, Hsd17b8, Sult1e1, } \\
\text { Akr1d1, Ugt2b37, Cyp2d10, Hsd17b6, Cyp2c22, Cyp2d26, } \\
\text { Ugt2b15, Ugt2b10, Cyp3a2, Cyp2d1, Hsd17b12, Cyp2c23, Cyp2c7, } \\
\text { Cyp2c13, Cyp2e1, Hsd11b1, Cyp2b3, Hsd3b5, Comt, Ugt2b17 }\end{array}$ \\
\hline $\begin{array}{l}\text { Ubiquinone and other terpenoid- } \\
\text { quinone biosynthesis }\end{array}$ & 12 & 4 & 0.333 & Vkorc111, Hpd, Coq5, Ggcx \\
\hline Biotin metabolism & 3 & 1 & 0.333 & Oxsm \\
\hline Pantothenate and CoA biosynthesis & 19 & 6 & 0.316 & Enpp3, Dpyd, Upb1, Dpys, Ppcs, Coasy \\
\hline Fatty acid elongation & 29 & 9 & 0.310 & Acaa2, Acot2, Acot4, Mecr, Hsd17b12, Hadha, Tecr, Echs1, Hadhb \\
\hline Alzheimer's disease & 182 & 56 & 0.308 & $\begin{array}{l}\text { Ndufs2, Ndufv2, Ndufa6, Uqcrc2, Hsd17b10, Ndufc2, Ndufs1, } \\
\text { Cox7a2, Cox4i1, Ndufb4, Ndufb5, Uqcr10, Ndufb10, Cox5b, } \\
\text { Sdhd, Ndufs8, Uqcrq, Ndufs7, Atp5f1, Ndufa8, Ndufa12, Ndufa5, } \\
\text { Ndufa4, Ide, Atp5o, Ndufv1, Uqcrc1, Apoe, Ndufa9, Atp5b, } \\
\text { Gapdh, Ndufab1, Ndufs4, Atp5j, Ndufs3, Ndufa2, Atp5d, Calm1, } \\
\text { Atp5h, Ndufa10, Lrp1, Ndufa7, Atp2a2, Ndufb7, Cox6c2, Uqcr11, } \\
\text { Cox6a1, Sdha, Cox6b1, Atp5a1, Ndufb3, Sdhc, Cyc1, Uqcrfs1, } \\
\text { Cox5a, Sdhb }\end{array}$ \\
\hline Terpenoid backbone biosynthesis & 23 & 7 & 0.304 & Acat2, Hmgcs1, Idi1, Fdps, Hmgcs2, Acat1, Pcyox1 \\
\hline
\end{tabular}


Supplementary Table 5 (Continued)

\begin{tabular}{|c|c|c|c|c|}
\hline KEGG Pathway ${ }^{\mathrm{A}}$ & Rat Genes ${ }^{\mathrm{B}}$ & SWATH Proteins ${ }^{\mathrm{C}}$ & Coverage $^{D}$ & List of Proteins Detected $^{\mathrm{E}}$ \\
\hline Huntington's disease & 204 & 62 & 0.304 & $\begin{array}{l}\text { Ndufs2, Ap2a1, Ndufv2, Ndufa6, Uqcrc2, Ndufc2, Ndufs1, Cox7a2, } \\
\text { Cox4i1, Ndufb4, Ndufb5, Uqcr10, Ndufb10, Cox5b, Sdhd, Ndufs8, } \\
\text { Uqcrq, Ndufs7, Atp5f1, Ndufa8, Ndufa12, Vdac2, Ndufa5, Tgm2, } \\
\text { Ndufa4, Atp5o, Ndufv1, Slc25a5, Uqcrc1, Ap2a2, Ndufa9, Atp5b, } \\
\text { Sod2, Ndufab1, Ndufs4, Atp5j, Ndufs3, Ndufa2, Atp5d, Ap2b1, } \\
\text { Atp5h, Ndufa10, Cltc, Ndufa7, Ndufb7, Cox6c2, Uqcr11, Cox6a1, } \\
\text { Sdha, Sod1, Vdac3, Ppif, Cox6b1, Atp5a1, Ndufb3, Vdac1, Gpx1, } \\
\text { Sdhc, Cyc1, Uqcrfs1, Cox5a, Sdhb }\end{array}$ \\
\hline
\end{tabular}

Non-alcoholic fatty liver disease (NAFLD)

\begin{abstract}
Steroid biosynthesis
Protein processing in endoplasmic
\end{abstract} reticulum

Cyanoamino acid metabolism

Ribosome

Starch and sucrose metabolism

Nicotinate and nicotinamide metabolism

Sulfur relay system

Fructose and mannose metabolism

Linoleic acid metabolism

Galactose metabolism

Amino sugar and nucleotide sugar metabolism

Riboavin metabolism

Valine, leucine and isoleucine biosynthesis

Cardiac muscle contraction

Aminoacyl-tRNA biosynthesis

Protein export

Fat digestion and absorption

Arachidonic acid metabolism

Bacterial invasion of epithelial cells

Bile secretion
20

168 7

172
Ndufs2, Ndufv2, Ndufa6, Uqcrc2, Ndufc2, Eif2s1, Ndufs1, Cox7a2, Cox4i1, Ndufb4, Ndufb5, Uqcr10, Ndufb10, Cox5b, Rac1, Sdhd, Cdc42, Ndufs8, Uqcrq, Ndufs7, Ndufa8, Ndufa12, Ndufa5, Ndufa4, Ndufv1, Uqcrc1, Ndufa9, Ndufab1, Ndufs4, Ndufs3, Ndufa2, Pklr, Ndufa10, Ndufa7, Ndufb7, Cox6c2, Uqcr11, Cyp2e1, Cox6a1, Sdha, Cox6b1, Ndufb3, Prkag1, Sec13, Sdhc, Cyc1, Uqcrfs1, Cox5a, Sdhb

Fdft1, Ebp, Dhcr7, Atad3, Nsdhl, Lipa

Erp29, Man1a1, Ighm, Eif2s1, Rrbp1, Sec61a1, Dnajb11, Hsp90b1, Hsph1, Dnajc3, Lman2, Dnaja2, Dnaja1, Canx, Vcp, Sar1b, Pdia3, Ssr4, Hsp90aa1, Ube2d3, Pdia6, Bcap31, Hspa5, Rad23b, Hspa8, Rpn1, A1cf, Pdia4, Stt3a, Ssr1, Ddost, Prkcsh, Stt3b, Sec23a, Hsp90ab1, Ns1c, Uggt1, Txndc5, Hyou1, Sec31a, Ssr3, Sec61b, Calr, Lman1, Ubqln1, Rpn2, Ganab, P4hb, Sec24a

Shmt1, Shmt2

Rpl26, Rpl23, Rps8, Rplp1, Rplp0, Rpl35, Rpl10a, Rpl17, Rpl21, Ste2, Rps12, Rps11, Rps5, Rpl27, Rpl8, Rps4x, Rps28, Rps3, Rpl39, Rps18, Rps16, Ckb, Rps26, Rps15, Rpl10, Rpl30, Rps23, Rpl3, Rps7, Rpl32, Rpl7, Rpl18a, Rpsa, Rpl12, Rpl5, Rps9, Rpl6, Rps6, Rpl24, Rpl15, Rpl13, Rpl7a, Rpl4, Rps3a, Rpl13a, Uba52, Rpl18, Rps21, Rpl11

Enpp3, Gys2, Ugt2b17, Ugt1a1, Pygl, Ugt2b37, Gusb, Ugt2b15, Ugt2b10, Ugdh, Ugp2, Gbe1, G6pc, Agl, Ugt2b17

Enpp3, Nnt, Nampt, Qprt, Aox1, Aox3, Pnp, Nadk2

\section{Nfs1, Tst, Mpst}

Aldoa, Akr1b1, Khk, Aldob, Sord, Fbp1, Pfkl, Gmppb, Tpi1, Gmppa

Cyp1a2, Cyp3a18, Pla2g12b, Cyp4f1, Сyp2c22, Сyp3a2, Сyp2c23, Сур2c7, Сур2c13, Сур2e1, Сур2j3

Glb1, Akr1b1, Gale, Galk1, Pfkl, Ugp2, Galm, G6pc

Hexa, Gne, Pgm3, Cyb5r3, Gale, Galk1, Ugdh, Nans, Ugp2, Gnpnat1, Gmppb, Gmppa

Blvrb

Sds

Uqcrc2, Cox7a2, Atp1b1, Cox4i1, Tpm3, Uqcr10, Atp1b3, Cox5b, Uqcrq, Tpm1, Uqcrc1, Atp1a1, Atp2a2, Cox6c2, Uqcr11, Cox6a1, Cox6b1, Cyc1, Uqcrfs1, Cox5a

Rars, Qars, Hars, Farsb, Iars2, Yars, Dars, Tars, Cars, Nars, Aars, Eprs, Farsa, Mars, Kars, Gars

Sec61a1, Hspa5, Srp72, Spcs3, Sec11a, Sec61b

Acat2, Pla2g12b, Cyp4f1, Fabp1, Apob, Apoa4, Mttp, Apoa1, Got2

Ephx2, Pla2g12b, Cyp4f1, Ptges3, Gpx3, Cyp2c22, Cyp4a14, Lta4h, Cyp2c23, Сyp2c7, Сyp2c13, Сyp2e1, Cyp4a2, Fahd2, Сyp2b3, Gpx1, Cyp2j3, Ptges2

Dnm2, Rac1, Arpc3, Cdc42, Rhoa, Itgb1, Fn1, Arpc5, Actb, Arpc4, Vcl, Ctnna1, H6pd, Ctnnb1, Arpc2, Cltc, Ilk

Slc27a5, Baat, Abcc2, Atp1b1, Abcb11, Slco1a4, Ndufs5, Ephx1, Atp1b3, Slc22a7, Prkaca, Ugt2b15, Atp1a1, Ca2, Sult2a1 
Supplementary Table 5 (Continued)

\begin{tabular}{|c|c|c|c|c|}
\hline KEGG Pathway ${ }^{A}$ & Rat Genes ${ }^{B}$ & SWATH Proteins ${ }^{\mathrm{C}}$ & Coverage $^{\mathrm{D}}$ & List of Proteins Detected ${ }^{\mathrm{E}}$ \\
\hline Thyroid hormone synthesis & 69 & 14 & 0.203 & $\begin{array}{l}\text { Iyd, Atp1b1, Hsp90b1, Gpx3, Atp1b3, Gsr, Canx, Prkaca, Hspa5, } \\
\text { Asgr2, Atp1a1, Pdia4, Asgr1, Gpx1 }\end{array}$ \\
\hline Thiamine metabolism & 5 & 1 & 0.200 & Nfs1 \\
\hline alpha-Linolenic acid metabolism & 25 & 5 & 0.200 & Pla2g12b, Cyp4f1, Acox1, Acaa1a, Acox3 \\
\hline Glycosaminoglycan degradation & 21 & 4 & 0.190 & Glb1, Hexa, Gusb, Naglu \\
\hline Collecting duct acid secretion & 27 & 5 & 0.185 & Atp6v1e1, Atp6v1f, Atp6v0d1, Ca2, Slc4a1 \\
\hline $\begin{array}{l}\text { Endocrine and other factor-regulated } \\
\text { calcium reabsorption }\end{array}$ & 49 & 9 & 0.184 & Ap2a1, Atp1b1, Dnm2, Atp1b3, Prkaca, Ap2a2, Atp1a1, Ap2b1, Cltc \\
\hline Glycerolipid metabolism & 60 & 11 & 0.183 & $\begin{array}{l}\text { Gk, Aldh9a1, Hspa9, Aldh3a2, Mgll, Akr1b1, Aldh1b1, Aldh7a1, } \\
\text { Glyctk, Akr1a1, Tkfc }\end{array}$ \\
\hline Taurine and hypotaurine metabolism & 11 & 2 & 0.182 & Baat, Csad \\
\hline N-Glycan biosynthesis & 50 & 9 & 0.180 & Man1a1, Ighm, Rpn1, Alcf, Stt3a, Ddost, Stt3b, Rpn2, Ganab \\
\hline Glucagon signaling pathway & 102 & 18 & 0.176 & $\begin{array}{l}\text { Ldha, Gys2, Pygl, Pck2, Pdhb, Pck1, Acaca, Prkaca, Pdha1, Fbp1, } \\
\text { Pfkl, Calm1, Cpt1a, G6pc, Prkag1, Sec13, Pgam1, Slc2a2 }\end{array}$ \\
\hline Adherens junction & 74 & 12 & 0.162 & $\begin{array}{l}\text { Actn1, Actn4, Rac1, Cdc42, Rhoa, Actb, Ctnnd1, Acp1, Vcl, Ctnna1, } \\
\text { H6pd, Ctnnb1 }\end{array}$ \\
\hline $\begin{array}{l}\text { Arrhythmogenic right ventricular } \\
\text { cardiomyopathy (ARVC) }\end{array}$ & 74 & 12 & 0.162 & $\begin{array}{l}\text { Actn1, Actn4, Itgb1, Jup, Actb, Itgb3, Ctnna1, H6pd, Ctnnb1, } \\
\text { Atp2a2, Lmna, Itga1 }\end{array}$ \\
\hline $\begin{array}{l}\text { Complement and coagulation } \\
\text { cascades }\end{array}$ & 82 & 13 & 0.159 & $\begin{array}{l}\text { Map1, Fgg, Mbl2, Serpina1, C4a, Serping1, Fga, Serpinc1, Fgb, Vtn, } \\
\text { Serpinf2, Plg, Fh }\end{array}$ \\
\hline $\begin{array}{l}\text { Carbohydrate digestion and } \\
\text { absorption }\end{array}$ & 44 & 7 & 0.159 & Slc37a4, Atp1b1, Atp1b3, Atp1a1, G6pc, Slc2a2, Aldh4a1 \\
\hline Other glycan degradation & 19 & 3 & 0.158 & Glb1, Hexa, Man2c1 \\
\hline Lysosome & 129 & 20 & 0.155 & $\begin{array}{l}\text { Glb1, Hexa, Scarb2, Tpp1, Ctsc, Psap, Ctsh, Gusb, Ctsb, Asah1, } \\
\text { Lamp2, Atp6v0d1, Naga, Naglu, Ctsa, Ap1b1, Cltc, Gm2a, Lipa, } \\
\text { Ctsd }\end{array}$ \\
\hline AMPK signaling pathway & 129 & 20 & 0.155 & $\begin{array}{l}\text { Gys2, Rab11b, Pck2, Mlycd, Pck1, Acaca, Fasn, Rab10, Cyp2c6, } \\
\text { Cab39, Rab2a, Fbp1, Rab14, Eef2, Pfkl, Ppp2r1a, Cpt1a, G6pc, } \\
\text { Prkag1, Sec13 }\end{array}$ \\
\hline Folate biosynthesis & 13 & 2 & 0.154 & Spr, Qdpr \\
\hline RNA transport & 164 & 25 & 0.152 & $\begin{array}{l}\text { Eif4a2, Eif3c, Eif2s1, Eif3d, Eif2s3y, Eif3b, Eif3a, Eif5, Xpo1, Eif4g1, } \\
\text { Sumo3, Rangap1, Pabpc1, Eif3g, Kpnb1, Eif3j, Eif3i, Ddx39b, } \\
\text { Eif3f, Eif4a1, Upf1, Eif4a3, Ran, Eef1a1, Cyfip1 }\end{array}$ \\
\hline Synaptic vesicle cycle & 62 & 9 & 0.145 & $\begin{array}{l}\text { Ap2a1, Atp6v1e1, Dnm2, Atp6v1f, Atp6v0d1, Ap2a2, Ap2b1, Cltc, } \\
\text { Napa }\end{array}$ \\
\hline $\begin{array}{l}\text { Vasopressin-regulated water } \\
\text { reabsorption }\end{array}$ & 43 & 6 & 0.140 & Rab5c, Rab11b, Rab5b, Prkaca, Arhgdia, Dync1h1 \\
\hline Central carbon metabolism in cancer & 65 & 9 & 0.138 & Ldha, Gls2, Pdhb, Pdha1, Sirt3, Isoc2, Pfkl, Pgam1, Slc2a2 \\
\hline Insulin resistance & 111 & 15 & 0.135 & $\begin{array}{l}\text { Slc27a5, Gys2, Pygl, Pck2, Ppp2r4, Pck1, Slc27a2, Sars, Ppp1ca, } \\
\text { Cpt1a, G6pc, Stat3, Prkag1, Sec13, Slc2a2 }\end{array}$ \\
\hline $\begin{array}{l}\text { Glycosphingolipid } \\
\text { biosynthesis - ganglio series }\end{array}$ & 15 & 2 & 0.133 & Glb1, Hexa \\
\hline $\begin{array}{l}\text { Glycosphingolipid biosynthesis - globo } \\
\text { series }\end{array}$ & 15 & 2 & 0.133 & Hexa, Naga \\
\hline $\begin{array}{l}\text { Leukocyte transendothelial } \\
\text { migration }\end{array}$ & 120 & 16 & 0.133 & $\begin{array}{l}\text { Actn1, Msn, Actn4, Rac1, Cdc42, Rhoa, Itgb1, F11r, Actb, Myl12b, } \\
\text { Ctnnd1, Vcl, Ctnna1, H6pd, Ctnnb1, Rap1b }\end{array}$ \\
\hline Purine metabolism & 181 & 24 & 0.133 & $\begin{array}{l}\text { Enpp3, Nme2, Adk, Adsl, Nme1, RGD1309350, Uox, Hprt1, Gda, } \\
\text { Itpa, Ak3, Paics, Nme3, Entpd5, Dguok, Atic, Pklr, Pnp, Aprt, Ak2, } \\
\text { Papss2, Xdh, Urad, Ak4 }\end{array}$ \\
\hline Adipocytokine signaling pathway & 75 & 10 & 0.133 & Pck2, Pck1, Acsl1, Acsl4, Cpt1a, G6pc, Stat3, Prkag1, Sec13, Acs15 \\
\hline Antigen processing and presentation & 98 & 13 & 0.133 & $\begin{array}{l}\text { Ctsb, Psme1, Hadh, Hspa4, Canx, Pdia3, Hsp90aa1, Hspa5, Hspa8, } \\
\text { RT1-A2, Psme2, Hsp90ab1, Calr }\end{array}$ \\
\hline
\end{tabular}


Supplementary Table 5 (Continued)

\begin{tabular}{|c|c|c|c|c|}
\hline KEGG Pathway ${ }^{\mathrm{A}}$ & Rat Genes ${ }^{\mathrm{B}}$ & SWATH Proteins ${ }^{\mathrm{C}}$ & Coverage $^{\mathrm{D}}$ & List of Proteins Detected ${ }^{\mathrm{E}}$ \\
\hline Salmonella infection & 83 & 11 & 0.133 & $\begin{array}{l}\text { Rac1, Arpc3, Cdc42, Pfn1, Flnb, Arpc5, Actb, Arpc4, Arpc2, Rab7a, } \\
\text { Dync1h1 }\end{array}$ \\
\hline Pyrimidine metabolism & 106 & 14 & 0.132 & $\begin{array}{l}\text { Tymp, Txnrd2, Nme2, Nme1, Dpyd, Upb1, Dpys, Itpa, Nme3, } \\
\text { Entpd5, Umps, Txnrd1, Pnp, Cmpk1 }\end{array}$ \\
\hline Phagosome & 198 & 26 & 0.131 & $\begin{array}{l}\text { Tfrc, Mbl2, Atp6v1e1, Tubb4b, Vamp3, Sec61a1, Rab5c, Atp6v1f, } \\
\text { Rab5b, Rac1, Tuba4a, Canx, Lamp2, Itgb1, Atp6v0d1, Sec22b, } \\
\text { Actb, Itgb3, RT1-A2, Rab7a, Dync1h1, Sec61b, Calr, Tubb5, Dhfr, } \\
\text { Tubb2a }\end{array}$ \\
\hline $\mathrm{ABC}$ transporters & 47 & 6 & 0.128 & Abcc2, Abca6, Abcb11, Abcd3, Abcc6, Abca8a \\
\hline Epstein-Barr virus infection & 229 & 29 & 0.127 & $\begin{array}{l}\text { Vim, Ywhab, Psmd7, Snd1, Psmd13, Psmd14, Psmc2, Xpo1, Psmd12, } \\
\text { Psmd1, Prkaca, Psmc1, Psmd11, Hspa8, Ywhag, Ywhaz, Psmd3, } \\
\text { Psmc5, Psmd8, RT1-A2, Ywhah, Stat3, Psmc6, Psmc4, Ddx58, } \\
\text { Ran, Ywhae, Psmc3, Psmd2 }\end{array}$ \\
\hline Renin-angiotensin system & 32 & 4 & 0.125 & Prep, Anpep, Ctsa, Nln \\
\hline Vitamin digestion and absorption & 24 & 3 & 0.125 & Apob, Apoa4, Apoa1 \\
\hline Endocytosis & 288 & 35 & 0.122 & $\begin{array}{l}\text { Tfrc, Ap2a1, Snx2, Snx5, Dnm2, Arf6, Rab5c, Rab11b, Rab5b, Arpc3, } \\
\text { Cdc42, Vps26b, Vps4b, Capza2, Vps25, Rhoa, Pdcd6ip, Rab10, } \\
\text { Cyp2c6, Capza1, Arpc5, Arf3, Ap2a2, Hspa8, Arpc4, Ap2b1, } \\
\text { Arpc2, Cltc, RT1-A2, Rab7a, Vps35, Arfgap2, Vps29, Ehd3, Capzb }\end{array}$ \\
\hline Legionellosis & 58 & 7 & 0.121 & Eef1g, Ggct, Vcp, Sar1b, Sec22b, Hspa8, Eef1a1 \\
\hline HIF-1 signaling pathway & 109 & 13 & 0.119 & $\begin{array}{l}\text { Tfrc, Ldha, Tceb2, Aldoa, Pdhb, Cul2, Pdha1, Gapdh, Pfkl, Eno1, } \\
\text { Stat3, Rps6, Pgk1 }\end{array}$ \\
\hline $\begin{array}{l}\text { Hypertrophic cardiomyopathy } \\
\text { (HCM) }\end{array}$ & 85 & 10 & 0.118 & Tpm3, Itgb1, Tpm1, Actb, Itgb3, Atp2a2, Lmna, Prkag1, Sec13, Itga1 \\
\hline Prion diseases & 34 & 4 & 0.118 & Prkaca, Hspa5, Stip1, Sod1 \\
\hline Ether lipid metabolism & 44 & 5 & 0.114 & Tmem86b, Pla2g12b, Cyp4f1, Cept1, Pafah1b2 \\
\hline Insulin signaling pathway & 140 & 16 & 0.114 & $\begin{array}{l}\text { Gys2, Pygl, Pck2, Pck1, Acaca, Fasn, Prkaca, Fbp1, Calm1, Pklr, } \\
\text { Ppp1ca, G6pc, Prkar2a, Rps6, Prkag1, Sec13 }\end{array}$ \\
\hline Regulation of actin cytoskeleton & 221 & 25 & 0.113 & $\begin{array}{l}\text { Actn1, Msn, Actn4, Rac1, Arpc3, Cdc42, Rhoa, Itgb1, Iqgap2, Pfn1, } \\
\text { C2, Diaph1, Fn1, Arpc5, Actb, Arpc4, Myl12b, Itgb3, Vcl, Ppp1ca, } \\
\text { Arpc2, Rdx, Itga1, Cyfip1, Cfl1 }\end{array}$ \\
\hline Platelet activation & 127 & 14 & 0.110 & $\begin{array}{l}\text { Fgg, Fga, Rhoa, Itgb1, Prkaca, Stim1, Pbld, Fgb, Actb, Myl12b, Itgb3, } \\
\text { Ppplca, Tln1, Rap1b }\end{array}$ \\
\hline Fc gamma R-mediated phagocytosis & 91 & 10 & 0.110 & Dnm2, Arf6, Rac1, Arpc3, Cdc42, Cfl2, Arpc5, Arpc4, Arpc2, Cfl1 \\
\hline Mineral absorption & 46 & 5 & 0.109 & Fth1, Atp1b1, Atp1b3, Atp1a1, Atox1 \\
\hline Spliceosome & 137 & 15 & 0.109 & $\begin{array}{l}\text { Snrpd3, Snrpd1, Hnrnpa1, Ddx5, Hnrnpk, U2af2, Hspa8, Ddx39b, } \\
\text { Snrpa1, Srsf1, Hnrnpa3, Hnrnpu, Hnrnpm, Eif4a3, Tra2b }\end{array}$ \\
\hline mRNA surveillance pathway & 93 & 10 & 0.108 & $\begin{array}{l}\text { Gspt1, Cpsf6, Dazap1, Etf1, Pabpc1, Ddx39b, Ppp2r1a, Ppp1ca, Upf1, } \\
\text { Eif4a3 }\end{array}$ \\
\hline Focal adhesion & 206 & 22 & 0.107 & $\begin{array}{l}\text { Actn1, Col6a1, Actn4, Rac1, Cdc42, Rhoa, Itgb1, Flnb, Diaph1, Fn1, } \\
\text { Actb, Myl12b, Vtn, Itgb3, Parva, Vcl, Ctnnb1, Ppp1ca, Tln1, Ilk, } \\
\text { Rap1b, Itga1 }\end{array}$ \\
\hline Apoptosis & 141 & 15 & 0.106 & $\begin{array}{l}\text { Ctsc, Eif2s1, Casp6, Ctsh, Ctsb, Tuba4a, Htra2, Actb, Parp3, Sptan1, } \\
\text { Lmnb1, Endog, Lmna, Aifm1, Ctsd }\end{array}$ \\
\hline Vascular smooth muscle contraction & 123 & 13 & 0.106 & $\begin{array}{l}\text { Pla2g12b, Cyp4f1, Myh11, Rhoa, Prkaca, Cyp4a14, Acta2, Calm1, } \\
\text { Ppp1ca, Cyp4a2, Fahd2, Myl6, Cald1 }\end{array}$ \\
\hline Tight junction & 143 & 15 & 0.105 & $\begin{array}{l}\text { Actn1, Actn4, Myh11, Cdc42, Rhoa, F11r, Actb, Myl12b, Ctnna1, } \\
\text { H6pd, Sptan1, Vapa, Ppp2r1a, Ctnnb1, Myh9 }\end{array}$ \\
\hline Pancreatic secretion & 97 & 10 & 0.103 & $\begin{array}{l}\text { Pla2g12b, Cyp4f1, Atp1b1, Atp1b3, Rac1, Rhoa, Atp1a1, Atp2a2, } \\
\text { Ca2, Rap1b }\end{array}$ \\
\hline Thyroid cancer & 29 & 3 & 0.103 & Tpm3, Tfg, Ctnnb1 \\
\hline Dilated cardiomyopathy & 91 & 9 & 0.099 & Tpm3, Itgb1, Tpm1, Prkaca, Actb, Itgb3, Atp2a2, Lmna, Itga1 \\
\hline
\end{tabular}


Supplementary Table 5 (Continued)

\begin{tabular}{|c|c|c|c|c|}
\hline KEGG Pathway ${ }^{\mathrm{A}}$ & Rat Genes ${ }^{B}$ & SWATH Proteins ${ }^{\mathrm{C}}$ & Coverage $^{\mathrm{D}}$ & List of Proteins Detected ${ }^{\mathrm{E}}$ \\
\hline Viral carcinogenesis & 235 & 23 & 0.098 & $\begin{array}{l}\text { Actn1, Ddx3x, Actn4, Ywhab, Snd1, Rac1, Cdc42, Ddb1, Rhoa, } \\
\text { Hnrnpk, Atp6v0d1, Prkaca, Hist1h2bd, Psmc1, Dnaja3, Ywhag, } \\
\text { Ywhaz, Hist1h4b, RT1-A2, Ywhah, Stat3, Vdac3, Ywhae }\end{array}$ \\
\hline Pertussis & 73 & 7 & 0.096 & C4a, Serping1, Rhoa, Itgb1, Cfl2, Calm1, Cfl1 \\
\hline Serotonergic synapse & 125 & 12 & 0.096 & $\begin{array}{l}\text { Сур2d10, Сур2с22, Сур2d26, Prkaca, Сyp2d1, Gnb2, Сур2с23, } \\
\text { Сур2с7, Сур2с13, Maob, Сyp2j3, Maoa }\end{array}$ \\
\hline $\begin{array}{l}\text { Inammatory mediator regulation of } \\
\text { TRP channels }\end{array}$ & 115 & 11 & 0.096 & $\begin{array}{l}\text { Cyp2c22, Prkaca, Cyp4a14, Сyp2c23, Сyp2c7, Calm1, Сyp2c13, } \\
\text { Ppp1ca, Cyp4a2, Fahd2, Сyp2j3 }\end{array}$ \\
\hline Gastric acid secretion & 74 & 7 & 0.095 & Atp1b1, Atp1b3, Prkaca, Actb, Atp1a1, Calm1, Ca2 \\
\hline Proteoglycans in cancer & 203 & 19 & 0.094 & $\begin{array}{l}\text { Msn, Rac1, Cdc42, Ddx5, Rhoa, Itgb1, Prkaca, Flnb, Fn1, F2, Lum, } \\
\text { Actb, Vtn, Itgb3, Ctnnb1, Ppp1ca, Stat3, Rps6, Rdx }\end{array}$ \\
\hline $\begin{array}{l}\text { Longevity regulating } \\
\text { pathway - multiple species }\end{array}$ & 65 & 6 & 0.092 & Prkaca, Hspa8, Sod2, Sod1, Prkag1, Sec13 \\
\hline Staphylococcus aureus infection & 57 & 5 & 0.088 & Fgg, Mbl2, C4a, Plg, Fh \\
\hline Amoebiasis & 106 & 9 & 0.085 & Actn1, Actn4, Arg1, Rab5c, Rab5b, Prkaca, Fn1, Vcl, Rab7a \\
\hline Systemic lupus erythematosus & 135 & 11 & 0.081 & $\begin{array}{l}\text { Actn1, Snrpd3, Actn4, Snrpd1, C4a, H2afv, H2afy, Hist1h2bd, } \\
\text { Hist1h4b, H2afx, Ssb }\end{array}$ \\
\hline Oocyte meiosis & 113 & 9 & 0.080 & $\begin{array}{l}\text { Ywhab, Prkaca, Ywhag, Ywhaz, Calm1, Ppp2r1a, Ppp1ca, Ywhah, } \\
\text { Ywhae }\end{array}$ \\
\hline GABAergic synapse & 90 & 7 & 0.078 & Gphn, Nat1, Abat, Gls2, Prkaca, Gnb2, Glul \\
\hline Amphetamine addiction & 65 & 5 & 0.077 & Prkaca, Calm1, Ppp1ca, Maob, Maoa \\
\hline Endometrial cancer & 52 & 4 & 0.077 & Ctnna1, H6pd, Ctnnb1, Ilk \\
\hline Thyroid hormone signaling pathway & 119 & 9 & 0.076 & Atp1b1, Atp1b3, Prkaca, Actb, Stat1, Atp1a1, Itgb3, Ctnnb1, Atp2a2 \\
\hline African trypanosomiasis & 40 & 3 & 0.075 & Hba-a1, Apoal, Hbb \\
\hline Renal cell carcinoma & 68 & 5 & 0.074 & Tceb2, Rac1, Cdc42, Cul2, Rap1b \\
\hline Maturity onset diabetes of the young & 27 & 2 & 0.074 & Pklr, Slc2a2 \\
\hline $\begin{array}{l}\text { Aldosterone-regulated sodium } \\
\text { reabsorption }\end{array}$ & 41 & 3 & 0.073 & Atp1b1, Atp1b3, Atpla1 \\
\hline Estrogen signaling pathway & 96 & 7 & 0.073 & Hsp90b1, Hsp90aa1, Prkaca, Hspa8, Calm1, Hsp90ab1, Fkbp4 \\
\hline ECM-receptor interaction & 84 & 6 & 0.071 & Col6a1, Itgb1, Fn1, Vtn, Itgb3, Itgal \\
\hline Hippo signaling pathway & 156 & 11 & 0.071 & $\begin{array}{l}\text { Ywhab, Actb, Ywhag, Ywhaz, Ctnna1, H6pd, Ppp2r1a, Ctnnb1, } \\
\text { Ppp1ca, Ywhah, Ywhae }\end{array}$ \\
\hline Amyotrophic lateral sclerosis (ALS) & 56 & 4 & 0.071 & Rac1, Tomm40, Sod1, Gpx1 \\
\hline Influenza A & 171 & 12 & 0.070 & $\begin{array}{l}\text { Eif2s1, Dnajc3, Xpo1, Fdps, Actb, Stat1, Hspa8, Stat2, Ddx39b, Plg, } \\
\text { Ddx58, Vdac1 }\end{array}$ \\
\hline cGMP-PKG signaling pathway & 171 & 12 & 0.070 & $\begin{array}{l}\text { Atp1b1, Atp1b3, Rhoa, Vdac2, Slc25a5, Atp1a1, Calm1, Ppp1ca, } \\
\text { Atp2a2, Vdac3, Ppif, Vdac1 }\end{array}$ \\
\hline Ovarian steroidogenesis & 57 & 4 & 0.070 & Acot2, Prkaca, Hsd3b5, Сyp2j3 \\
\hline Gap junction & 88 & 6 & 0.068 & Tubb4b, Tuba4a, Prkaca, Tubb5, Dhfr, Tubb2a \\
\hline $\begin{array}{l}\text { Adrenergic signaling in } \\
\text { cardiomyocytes }\end{array}$ & 148 & 10 & 0.068 & $\begin{array}{l}\text { Atp1b1, Tpm3, Atp1b3, Tpm1, Prkaca, Atp1a1, Calm1, Ppp2r1a, } \\
\text { Ppp1ca, Atp2a2 }\end{array}$ \\
\hline Circadian rhythm & 30 & 2 & 0.067 & Prkag1, Sec13 \\
\hline Salivary secretion & 76 & 5 & 0.066 & Atp1b1, Atp1b3, Prkaca, Atp1a1, Calm1 \\
\hline Hepatitis B & 139 & 9 & 0.065 & Ddx3x, Ywhab, Ddb1, Stat1, Stat2, Ywhaz, Stat3, Vdac3, Ddx58 \\
\hline PI3K-Akt signaling pathway & 336 & 22 & 0.065 & $\begin{array}{l}\text { Col6a1, Ywhab, Gys2, Hsp90b1, Pck2, Rac1, Itgb1, Pck1, Hsp90aa1, } \\
\text { Fn1, Gnb2, Ywhag, Ywhaz, Vtn, Itgb3, Ppp2r1a, G6pc, Ywhah, } \\
\text { Rps6, Hsp90ab1, Ywhae, Itga1 }\end{array}$ \\
\hline Cocaine addiction & 46 & 3 & 0.065 & Prkaca, Maob, Maoa \\
\hline Glycerophospholipid metabolism & 95 & 6 & 0.063 & Gpd1, Pla2g12b, Cyp4f1, Lypla1, Cept1, Pcyt2 \\
\hline Pancreatic cancer & 65 & 4 & 0.062 & Rac1, Cdc42, Stat1, Stat3 \\
\hline Long-term potentiation & 65 & 4 & 0.062 & Prkaca, Calm1, Ppp1ca, Rap1b \\
\hline
\end{tabular}


Supplementary Table 5 (Continued)

\begin{tabular}{|c|c|c|c|c|}
\hline KEGG Pathway ${ }^{\mathrm{A}}$ & Rat Genes ${ }^{\mathrm{B}}$ & SWATH Proteins ${ }^{\mathrm{C}}$ & Coverage $^{\mathrm{D}}$ & List of Proteins Detected ${ }^{\mathrm{E}}$ \\
\hline Dopaminergic synapse & 130 & 8 & 0.062 & Prkaca, Gnb2, Calm1, Ppp2r1a, Ppp1ca, Maob, Maoa, Comt \\
\hline $\begin{array}{l}\text { SNARE interactions in vesicular } \\
\text { transport }\end{array}$ & 33 & 2 & 0.061 & Vamp3, Sec22b \\
\hline cAMP signaling pathway & 196 & 12 & 0.061 & $\begin{array}{l}\text { Atp1b1, Atp1b3, Rac1, Rhoa, Acox1, Prkaca, Atp1a1, Calm1, Ppp1ca, } \\
\text { Atp2a2, Acox3, Rap1b }\end{array}$ \\
\hline Alcoholism & 179 & 11 & 0.061 & $\begin{array}{l}\text { H2afv, Prkaca, H2afy, Hist1h2bd, Gnb2, Calm1, Ppp1ca, Hist1h4b, } \\
\text { Maob, H2afx, Maoa }\end{array}$ \\
\hline Measles & 138 & 8 & 0.058 & Msn, Eif2s1, Stat1, Hspa8, Stat2, Stat3, Ddx58, Gnb211 \\
\hline Insulin secretion & 86 & 5 & 0.058 & Atp1b1, Atp1b3, Prkaca, Atp1a1, Slc2a2 \\
\hline $\begin{array}{l}\text { AGE-RAGE signaling pathway in } \\
\text { diabetic complications }\end{array}$ & 104 & 6 & 0.058 & Rac1, Cdc42, Diaph1, Fn1, Stat1, Stat3 \\
\hline Toxoplasmosis & 123 & 7 & 0.057 & Irgm, Igtp, Itgb1, Stat1, Hspa8, Stat3, Ppif \\
\hline MicroRNAs in cancer & 143 & 8 & 0.056 & Vim, Gls2, Rhoa, Hnrnpk, Tpm1, Itgb3, Stat3, Rdx \\
\hline Prostate cancer & 89 & 5 & 0.056 & Hsp90b1, Hsp90aa1, Gstp1, Ctnnb1, Hsp90ab1 \\
\hline Rap1 signaling pathway & 216 & 12 & 0.056 & $\begin{array}{l}\text { Rac1, Cdc42, Rhoa, Itgb1, Pfn1, Actb, Ctnnd1, Itgb3, Calm1, } \\
\text { Ctnnb1, Tln1, Rap1b }\end{array}$ \\
\hline Neurotrophin signaling pathway & 126 & 7 & 0.056 & Rac1, Cdc42, Rhoa, Arhgdia, Calm1, Rap1b, Ywhae \\
\hline Oxytocin signaling pathway & 160 & 9 & 0.056 & Rhoa, Prkaca, Actb, Eef2, Calm1, Ppp1ca, Prkag1, Sec13, Myl6 \\
\hline Protein digestion and absorption & 93 & 5 & 0.054 & Col6a1, Atp1b1, Atp1b3, Dpp4, Atp1a1 \\
\hline Pathways in cancer & 400 & 21 & 0.053 & $\begin{array}{l}\text { Tceb2, Tpm3, Hsp90b1, Rac1, Cdc42, Rhoa, Itgb1, Cul2, Hsp90aa1, } \\
\text { Prkaca, Jup, Fn1, Tfg, Stat1, Ctbp1, Gnb2, Ctnna1, H6pd, Ctnnb1, } \\
\text { Stat3, Hsp90ab1 }\end{array}$ \\
\hline Calcium signaling pathway & 187 & 10 & 0.053 & $\begin{array}{l}\text { Vdac2, Prkaca, Slc25a5, Stim1, Pbld, Calm1, Atp2a2, Vdac3, Ppif, } \\
\text { Vdac1 }\end{array}$ \\
\hline Ras signaling pathway & 232 & 12 & 0.052 & $\begin{array}{l}\text { Pla2g12b, Cyp4f1, Arf6, Rab5c, Rab5b, Rac1, Cdc42, Rhoa, Prkaca, } \\
\text { Gnb2, Calm1, Rap1b }\end{array}$ \\
\hline NOD-like receptor signaling pathway & 58 & 3 & 0.052 & Hsp90b1, Hsp90aa1, Hsp90ab1 \\
\hline Malaria & 59 & 3 & 0.051 & Hba-a1, Hbb, Lrp1 \\
\hline Herpes simplex infection & 217 & 11 & 0.051 & $\begin{array}{l}\text { Eif2s1, Eef1d, Hnrnpk, Stat1, Stat2, Ppp1ca, Srsf1, RT1-A2, Ddx58, } \\
\text { Taf3, C1qbp }\end{array}$ \\
\hline FoxO signaling pathway & 136 & 7 & 0.051 & Pck2, Pck1, Sod2, G6pc, Stat3, Prkag1, Sec13 \\
\hline Chemokine signaling pathway & 178 & 9 & 0.051 & Rac1, Cdc42, Rhoa, Prkaca, Stat1, Gnb2, Stat2, Stat3, Rap1b \\
\hline Ubiquitin mediated proteolysis & 141 & 7 & 0.050 & Tceb2, Ube2n, Uba6, Ddb1, Cul2, Uba1, Ube2d3 \\
\hline Tuberculosis & 184 & 9 & 0.049 & Rab5c, Rab5b, Rhoa, Lamp2, Atp6v0d1, Stat1, Calm1, Rab7a, Ctsd \\
\hline RNA degradation & 82 & 4 & 0.049 & Pabpc1, Pfkl, Eno1, Wdr61 \\
\hline Hematopoietic cell lineage & 86 & 4 & 0.047 & Tfrc, Anpep, Itgb3, Itga1 \\
\hline $\begin{array}{l}\text { RIG-I-like receptor signaling } \\
\text { pathway }\end{array}$ & 64 & 3 & 0.047 & Ddx3x, Ddx58, Tkfc \\
\hline Hepatitis C & 129 & 6 & 0.047 & Eif2s1, Stat1, Stat2, Ppp2r1a, Stat3, Ddx58 \\
\hline Colorectal cancer & 64 & 3 & 0.047 & Rac1, Rhoa, Ctnnb1 \\
\hline Axon guidance & 130 & 6 & 0.046 & Rac1, Cdc42, Rhoa, Itgb1, Cfl2, Cfl1 \\
\hline Renin secretion & 67 & 3 & 0.045 & Ctsb, Prkaca, Calm1 \\
\hline HTLV-I infection & 293 & 13 & 0.044 & $\begin{array}{l}\text { Canx, Xpo1, Vdac2, Fdps, Prkaca, Slc25a5, Ctnnb1, RT1-A2, Vdac3, } \\
\text { Vdac1, Tln1, Ran, Calr }\end{array}$ \\
\hline Viral myocarditis & 93 & 4 & 0.043 & Rac1, Eif4g1, Actb, RT1-A2 \\
\hline Glutamatergic synapse & 115 & 5 & 0.043 & Nat1, Gls2, Prkaca, Gnb2, Glul \\
\hline Nucleotide excision repair & 47 & 2 & 0.043 & $\mathrm{Ddb} 1, \operatorname{Rad} 23 \mathrm{~b}$ \\
\hline $\begin{array}{l}\text { Longevity regulating } \\
\text { pathway - mammal }\end{array}$ & 94 & 4 & 0.043 & Prkaca, Sod2, Prkag1, Sec13 \\
\hline Mismatch repair & 23 & 1 & 0.043 & Ssbp1 \\
\hline
\end{tabular}


Supplementary Table 5 (Continued)

\begin{tabular}{|c|c|c|c|c|}
\hline KEGG Pathway ${ }^{\mathrm{A}}$ & Rat Genes ${ }^{B}$ & SWATH Proteins ${ }^{\mathrm{C}}$ & Coverage $^{\mathrm{D}}$ & List of Proteins Detected ${ }^{\mathrm{E}}$ \\
\hline Sphingolipid metabolism & 49 & 2 & 0.041 & Glb1, Asah1 \\
\hline Sphingolipid signaling pathway & 124 & 5 & 0.040 & Rac1, Asah1, Rhoa, Ppp2r1a, Ctsd \\
\hline Phototransduction & 25 & 1 & 0.040 & Calm1 \\
\hline Cell cycle & 127 & 5 & 0.039 & Ywhab, Ywhag, Ywhaz, Ywhah, Ywhae \\
\hline $\begin{array}{l}\text { Retrograde endocannabinoid } \\
\text { signaling }\end{array}$ & 103 & 4 & 0.039 & Mgll, Prkaca, Gnb2, Faah \\
\hline Prolactin signaling pathway & 76 & 3 & 0.039 & Stat1, Stat3, Slc2a2 \\
\hline Type II diabetes mellitus & 52 & 2 & 0.038 & Pklr, Slc2a2 \\
\hline Aldosterone synthesis and secretion & 84 & 3 & 0.036 & Prkaca, Calm1, Hsd3b5 \\
\hline Homologous recombination & 28 & 1 & 0.036 & Ssbp1 \\
\hline Acute myeloid leukemia & 57 & 2 & 0.035 & Jup, Stat3 \\
\hline Regulation of lipolysis in adipocytes & 59 & 2 & 0.034 & Mgll, Prkaca \\
\hline Wnt signaling pathway & 145 & 5 & 0.034 & Rac1, Rhoa, Prkaca, Ctbp1, Ctnnb1 \\
\hline Rheumatoid arthritis & 92 & 3 & 0.033 & Atp6vle1, Atp6v1f, Atp6v0d1 \\
\hline VEGF signaling pathway & 60 & 2 & 0.033 & Rac1, Cdc42 \\
\hline GnRH signaling pathway & 92 & 3 & 0.033 & Cdc42, Prkaca, Calm1 \\
\hline $\begin{array}{l}\text { Progesterone-mediated oocyte } \\
\text { maturation }\end{array}$ & 90 & 3 & 0.033 & Hsp90aa1, Prkaca, Hsp90ab1 \\
\hline Jak-STAT signaling pathway & 151 & 5 & 0.033 & Aox1, Stat1, Stat2, Aox3, Stat3 \\
\hline MAPK signaling pathway & 259 & 8 & 0.031 & Rac1, Cdc42, Prkaca, Ppm1b, Flnb, Ecsit, Hspa8, Rap1b \\
\hline Circadian entrainment & 97 & 3 & 0.031 & Prkaca, Gnb2, Calm1 \\
\hline mTOR signaling pathway & 64 & 2 & 0.031 & Cab39, Rps6 \\
\hline Inflammatory bowel disease (IBD) & 65 & 2 & 0.031 & Stat1, Stat3 \\
\hline Osteoclast dierentiation & 134 & 4 & 0.030 & Rac1, Stat1, Stat2, Itgb3 \\
\hline Melanogenesis & 99 & 3 & 0.030 & Prkaca, Calm1, Ctnnb1 \\
\hline DNA replication & 36 & 1 & 0.028 & Ssbp1 \\
\hline Leishmaniasis & 75 & 2 & 0.027 & Itgb1, Stat1 \\
\hline Inositol phosphate metabolism & 74 & 2 & 0.027 & Aldh6a1, Tpi1 \\
\hline Base excision repair & 37 & 1 & 0.027 & Parp3 \\
\hline Bladder cancer & 40 & 1 & 0.025 & Tymp \\
\hline TGF-beta signaling pathway & 86 & 2 & 0.023 & Rhoa, Ppp2r1a \\
\hline Ribosome biogenesis in eukaryotes & 88 & 2 & 0.023 & Xpol, Ran \\
\hline Small cell lung cancer & 88 & 2 & 0.023 & Itgb1, Fn1 \\
\hline Taste transduction & 89 & 2 & 0.022 & Prkaca, Aldh4a1 \\
\hline Morphine addiction & 92 & 2 & 0.022 & Prkaca, Gnb2 \\
\hline Basal transcription factors & 45 & 1 & 0.022 & Taf3 \\
\hline Toll-like receptor signaling pathway & 97 & 2 & 0.021 & Rac1, Stat1 \\
\hline Hedgehog signaling pathway & 47 & 1 & 0.021 & Prkaca \\
\hline Phospholipase D signaling pathway & 148 & 3 & 0.020 & Dnm2, Arf6, Rhoa \\
\hline Choline metabolism in cancer & 101 & 2 & 0.020 & Lypla1, Rac1 \\
\hline $\begin{array}{l}\text { Intestinal immune network for IgA } \\
\text { production }\end{array}$ & 49 & 1 & 0.020 & Pigr \\
\hline Notch signaling pathway & 52 & 1 & 0.019 & Ctbp1 \\
\hline $\begin{array}{l}\text { Chagas disease (American } \\
\text { trypanosomiasis) }\end{array}$ & 107 & 2 & 0.019 & Ppp2r1a, Calr \\
\hline $\mathrm{T}$ cell receptor signaling pathway & 109 & 2 & 0.018 & Cdc42, Rhoa \\
\hline Cholinergic synapse & 112 & 2 & 0.018 & Prkaca, Gnb2 \\
\hline TNF signaling pathway & 110 & 2 & 0.018 & Dnm1l, Hpx \\
\hline
\end{tabular}


Supplementary Table 5 (Continued)

\begin{tabular}{|c|c|c|c|c|}
\hline KEGG Pathway ${ }^{\mathrm{A}}$ & Rat Genes ${ }^{\mathrm{B}}$ & SWATH Proteins ${ }^{\mathrm{C}}$ & Coverage $^{\mathrm{D}}$ & List of Proteins Detected ${ }^{\mathrm{E}}$ \\
\hline Basal cell carcinoma & 55 & 1 & 0.018 & Ctnnb1 \\
\hline Cytosolic DNA-sensing pathway & 56 & 1 & 0.018 & Ddx58 \\
\hline $\begin{array}{l}\text { Transcriptional misregulation in } \\
\text { cancer }\end{array}$ & 174 & 3 & 0.017 & Fus, Ddx5, Jup \\
\hline Cell adhesion molecules (CAMs) & 172 & 3 & 0.017 & Itgb1, F11r, RT1-A2 \\
\hline Long-term depression & 62 & 1 & 0.016 & Ppp2r1a \\
\hline Glioma & 65 & 1 & 0.015 & Calm1 \\
\hline Graft-versus-host disease & 68 & 1 & 0.015 & RT1-A2 \\
\hline B cell receptor signaling pathway & 74 & 1 & 0.014 & Rac1 \\
\hline Fc epsilon RI signaling pathway & 70 & 1 & 0.014 & Rac1 \\
\hline $\begin{array}{l}\text { Signaling pathways regulating } \\
\text { pluripotency of stem cells }\end{array}$ & 142 & 2 & 0.014 & Ctnnb1, Stat3 \\
\hline Allograft rejection & 72 & 1 & 0.014 & RT1-A2 \\
\hline Chronic myeloid leukemia & 76 & 1 & 0.013 & Ctbpl \\
\hline Type I diabetes mellitus & 77 & 1 & 0.013 & RT1-A2 \\
\hline Autoimmune thyroid disease & 79 & 1 & 0.013 & RT1-A2 \\
\hline NF-kappa B signaling pathway & 95 & 1 & 0.011 & Ddx58 \\
\hline $\begin{array}{l}\text { Natural killer cell mediated } \\
\text { cytotoxicity }\end{array}$ & 101 & 1 & 0.010 & Racl \\
\hline $\begin{array}{l}\text { Phosphatidylinositol signaling } \\
\text { system }\end{array}$ & 96 & 1 & 0.010 & Calm 1 \\
\hline $\begin{array}{l}\text { Neuroactive ligand-receptor } \\
\text { interaction }\end{array}$ & 292 & 1 & 0.003 & $\mathrm{Plg}$ \\
\hline Olfactory transduction & 1249 & 2 & 0.002 & Prkaca, Calm1 \\
\hline
\end{tabular}


Statistically Significant Proteins (SSPs)

\begin{tabular}{ll} 
Reaction & Gene \\
\hline R2859 & Cyp2a2, \\
& Cyp1a2 \\
R2886 & Cyp2a2, \\
& Cyp1a2 \\
R3423 & Fdft1 \\
R3972 & Fdft1 \\
R6372 & Cyp2a2, \\
& Cyp1a2 \\
R6658 & Fmo1 \\
R6687 & Cyp2a2, \\
& Cyp1a2
\end{tabular}

Statistically Significant Metabolites (SSMs)

Metabolite ID Metabolite Name

nadph $[\mathrm{r}] \quad$ Nicotinamide adenine dinucleotide phosphate - reduced

frdp $[\mathrm{r}] \quad$ Farnesyl diphosphate

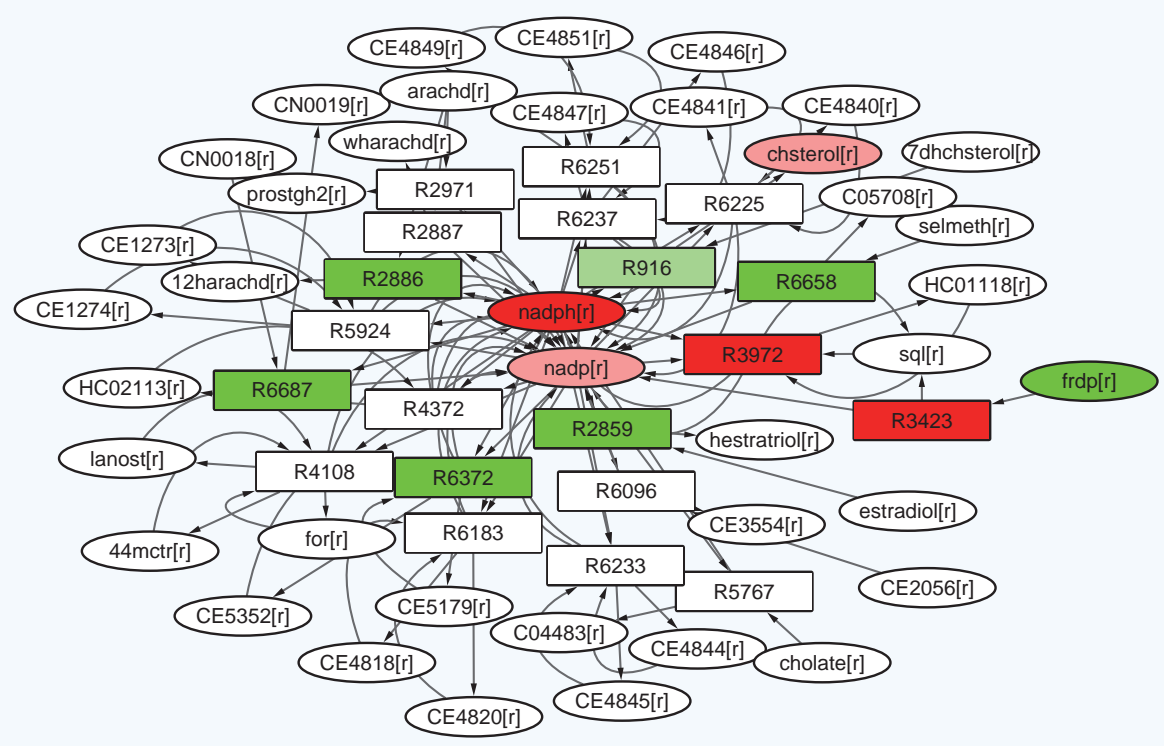

Supplementary Figure 1 The benefit of our algorithm was to identify co-factor driven interactions. In this module, we observed a significant up-regulation of the first specific enzyme of cholesterol biosynthesis, Farnesyl-Diphosphate Farnesyltransferase 1. Cholesterol biosynthesis is driven by the cofactor NADPH, which we also observe a significant upregulation of. 
Statistically Significant Proteins (SSPs)

\begin{tabular}{ll} 
Reaction & Gene \\
\hline R1772 & Ftcd \\
R2080 & Ftcd \\
R2329 & Aldh9a1, \\
& Aldh3a2 \\
R2602 & Mthfd1 \\
R2603 & Mthfd1 \\
R6367 & Cyp2a2, \\
& Cyp1a2 \\
R6406 & Aldh9a1, \\
& Aldh3a2 \\
R6408 & Aldh9a1, \\
& Aldh3a2
\end{tabular}

Statistically Significant Metabolites (SSMs)

Metabolite ID Metabolite Name nadph $[\mathrm{c}] \quad$ Nicotinamide adenine dinucleotide phosphate - reduced coa $[\mathrm{c}] \quad$ Coenzyme A

$\operatorname{atp}[\mathrm{c}] \quad$ ATP

$\operatorname{adp}[\mathrm{c}] \quad \mathrm{ADP}$

glu-L[c] L-glutamate(1-)

$\operatorname{dhf}[\mathrm{c}] \quad$ 7,8-Dihydrofolate

im4ac $[\mathrm{c}] \quad$ imidazol-4-ylacetate

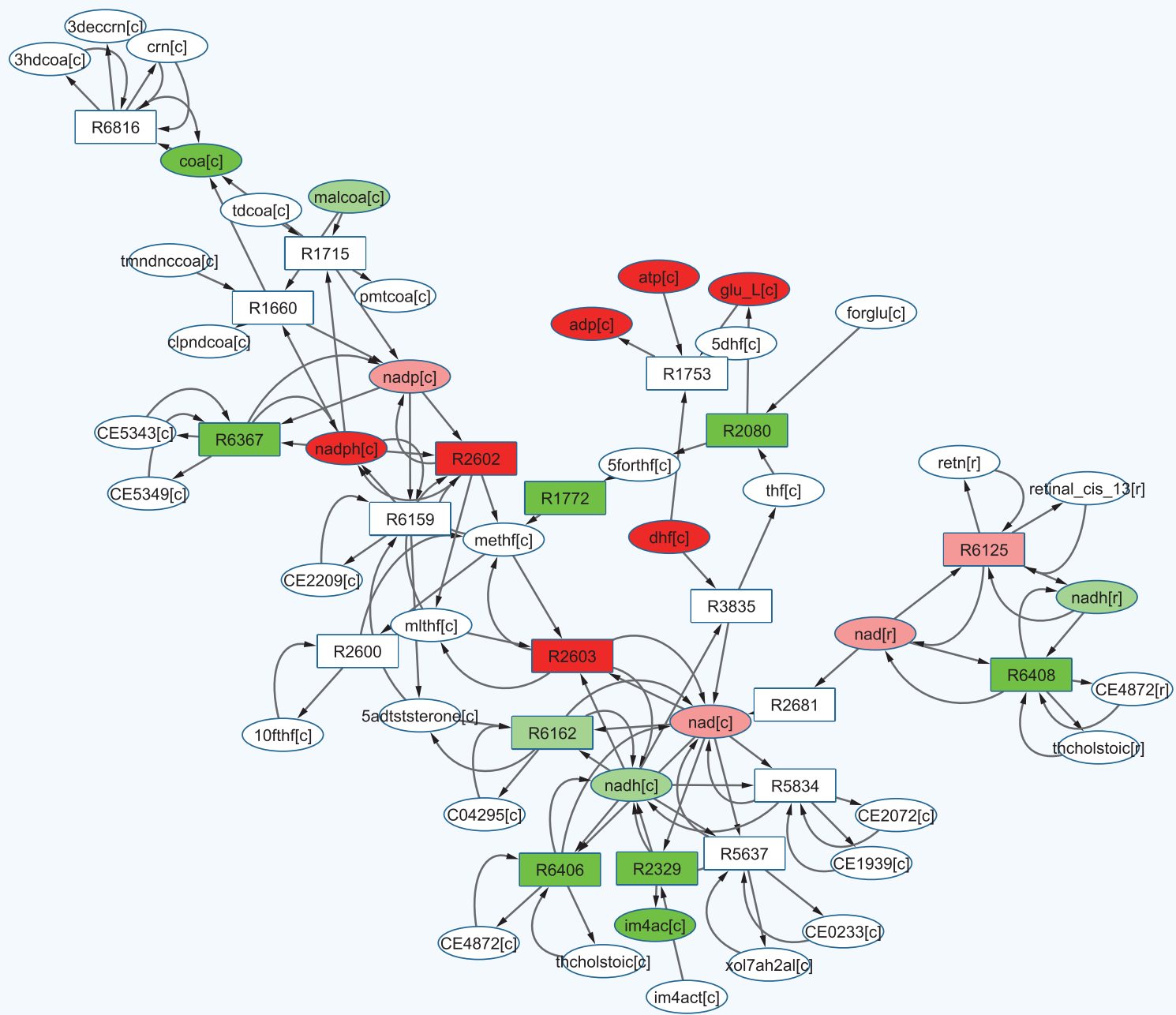

Supplementary Figure 2 It has been well documented in human and rodent models of RYGB alike that folate deficiency occurs after $\mathrm{RYGB}^{580}$. Here we observe an upregulation of Formyltetrahydrofolate Synthetase 1(MTHFD1) cofactors and a down-regulation of the protein itself suggesting this may be a key protein involved in the observed phenotype of folate deficiency after RYGB. 
Statistically Significant Proteins (SSPs)

\begin{tabular}{ll} 
Reaction & Gene \\
\hline R270 & Adk \\
R505 & Got1 \\
R2107 & Gk \\
R2714 & Nme2 \\
R2717 & Nme2 \\
R2720 & Nme2 \\
R2735 & Nme2
\end{tabular}

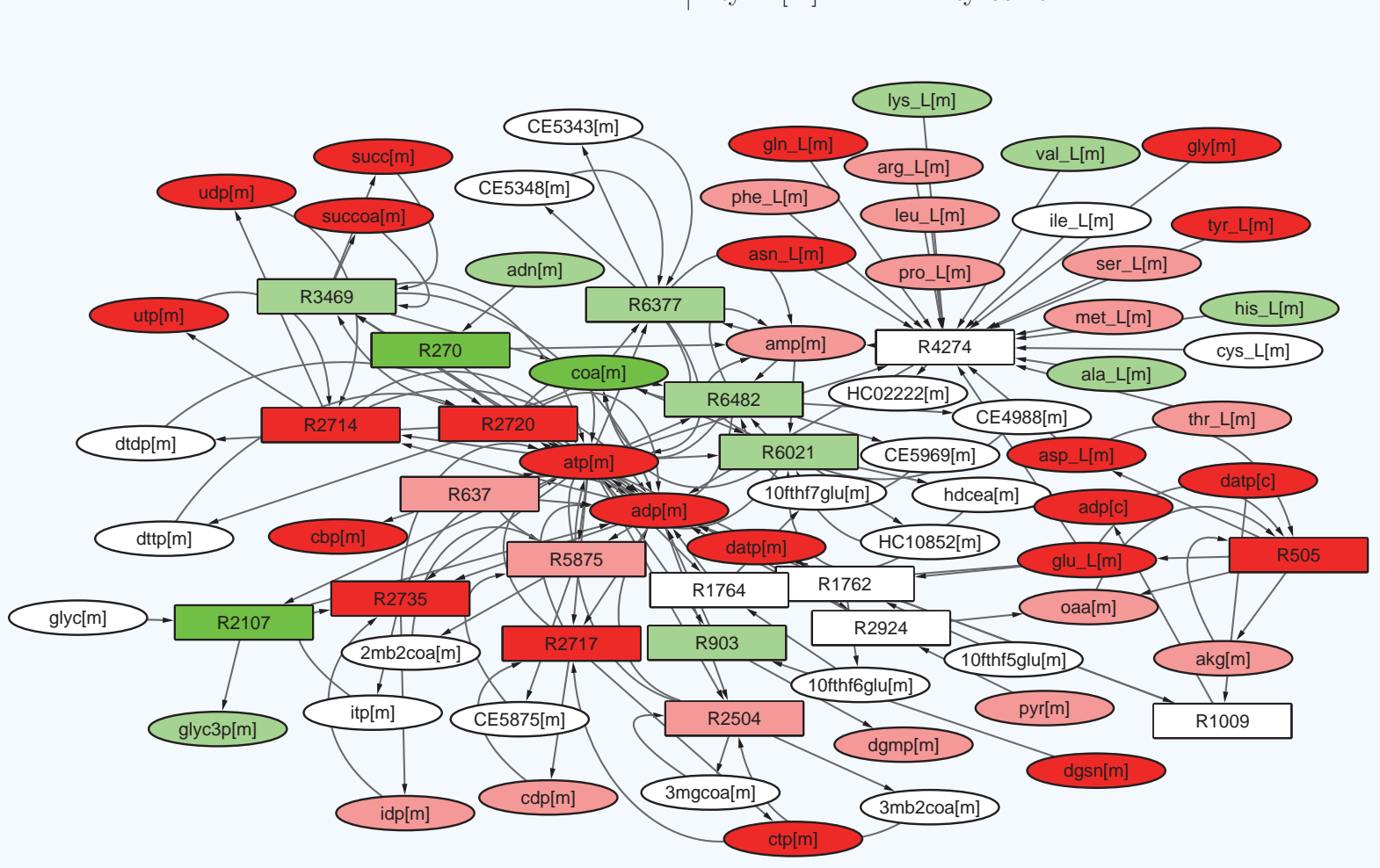

Supplementary Figure 3 Here, we observed a significant increase of cofactor driven production of Nucleoside diphosphate kinase 2 (Nme2) which is directly involved in pyrmidine metabolism. Previous work from our lab has shown increased production of purine and pyrimidine metabolites from the small intestine after RYGB surgery ${ }^{10}$.
Statistically Significant Metabolites (SSMs)

Metabolite ID Metabolite Name

$\operatorname{atp}[\mathrm{m}] \quad$ ATP

$\operatorname{adp}[\mathrm{m}] \quad$ ADP

glu_L[m] L-glutamate(1-)

asp_L[m] L-aspartate(1-)

cbp[m] Carbamoyl phosphate

dgsn $[\mathrm{m}] \quad$ Deoxyguanosine

$\operatorname{adp}[\mathrm{c}] \quad \mathrm{ADP}$

$\operatorname{datp}[\mathrm{c}] \quad \mathrm{dATP}$

$\mathrm{udp}[\mathrm{m}] \quad$ UDP

utp $[\mathrm{m}] \quad$ UTP

$\operatorname{ctp}[\mathrm{m}] \quad$ CTP

succoa $[\mathrm{m}] \quad$ Succinyl-CoA

succ $[\mathrm{m}] \quad$ Succinate

gly $[\mathrm{m}] \quad$ Glycine

asn_L[m] L-asparagine

$\operatorname{gln} \_\mathrm{L}[\mathrm{m}] \quad$ L-glutamine

tyr_L $[\mathrm{m}] \quad$ L-tyrosine $\operatorname{datp}[\mathrm{m}] \quad \mathrm{dATP}$

coa[m] Coenzyme A 
Statistically Significant Proteins (SSPs)

\begin{tabular}{ll} 
Reaction & Gene \\
\hline R84 & Got1 \\
R240 & Aco2 \\
R2313 & Idh2 \\
R2365 & Aadat \\
R2380 & Got1 \\
R3904 & Idh2 \\
R3922 & Aadat
\end{tabular}

Statistically Significant Metabolites (SSMs)

Metabolite ID Metabolite Name
glu_L[c] L-glutamate(1-)

glu_L[m] L-glutamate(1-)

4abut $[\mathrm{m}] \quad$ 4-Aminobutanoate $\operatorname{atp}[\mathrm{c}] \quad$ ATP

$\operatorname{adp}[\mathrm{c}] \quad$ ADP

nadph $[\mathrm{m}] \quad$ Nicotinamide adenine dinucleotide phosphate - reduced

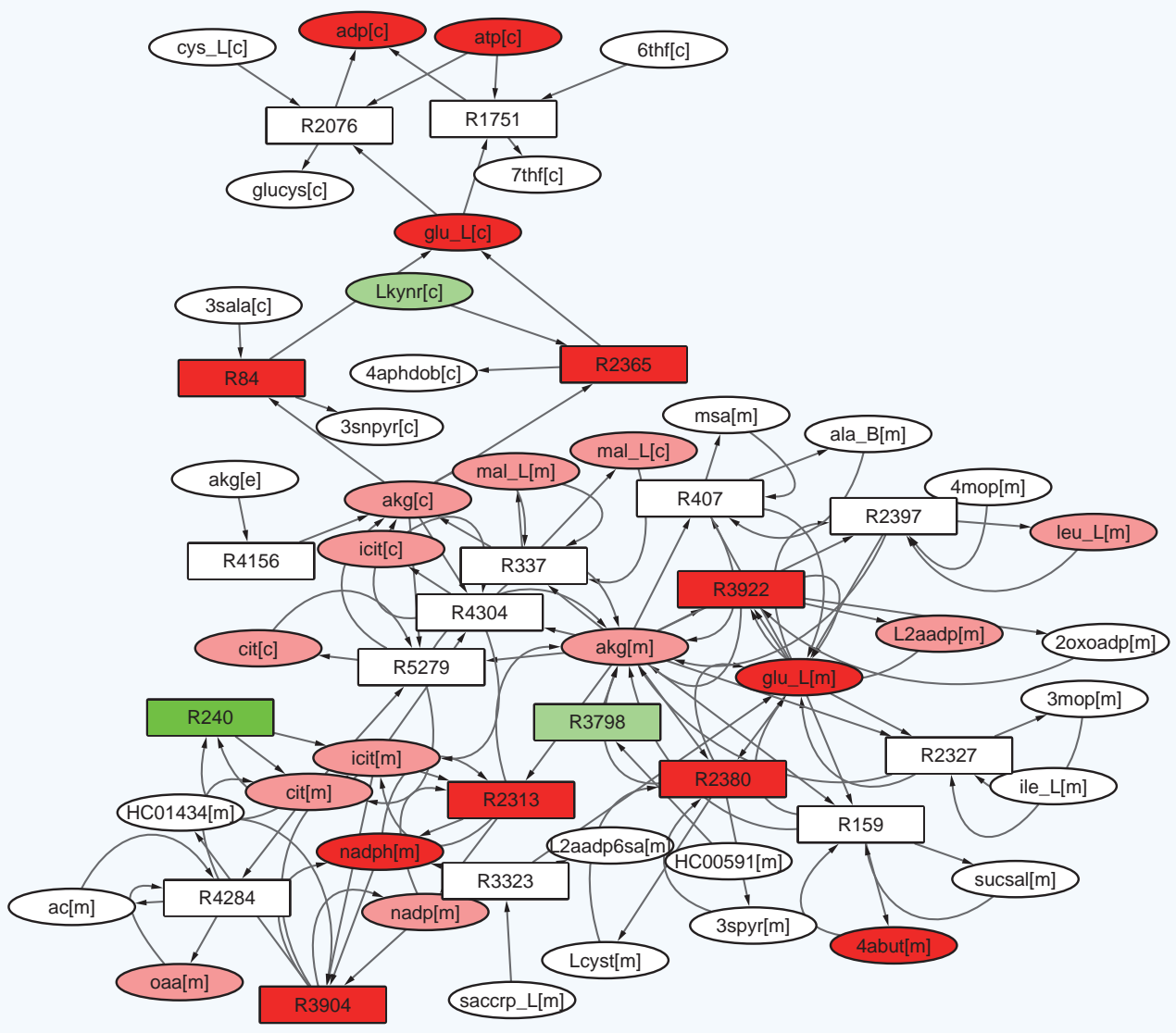

Supplementary Figure 4 The vast majority of metabolites and proteins of the TCA cycle are up-regulated. Interestingly, isocitrate dehydrogenase II, which is responsible for catalyzing the production of alpha ketoglutarate from 2-oxoglutarate is significantly upregulated. As TCA cycle metabolism is increased, this may lead to a significant increase in ATP generation potentially contributing to the accelerated metabolic phenotype observed after RYGB. Furthermore results indicate a significant upregulation of glutamate, which is an essential excitatory neurotransmitter which may be responsible for changes we observed in neuronal signaling pathways. 
Statistically Significant Proteins (SSPs)

\begin{tabular}{ll} 
Reaction & Gene \\
\hline R166 & Acat2 \\
R168 & Acat2 \\
R2666 & Ndufs2, \\
& Ndufv2 \\
R4007 & Aldh9a1, \\
& Aldh3a2 \\
R5420 & Baat \\
R5684 & Baat \\
R6407 & Aldh9a1, \\
& Aldh3a2
\end{tabular}

Statistically Significant Metabolites (SSMs)

\begin{tabular}{ll} 
Metabolite ID & Metabolite Name \\
\hline coa $[\mathrm{m}]$ & Coenzyme A \\
$\operatorname{accoa}[\mathrm{m}]$ & Acetyl-CoA \\
$\operatorname{atp}[\mathrm{m}]$ & ATP \\
$\operatorname{adp}[\mathrm{m}]$ & ADP
\end{tabular}

nadph $[\mathrm{m}] \quad$ Nicotinamide adenine dinucleotide phosphate - reduced fad $[\mathrm{m}] \quad$ Flavin adenine dinucleotide oxidized

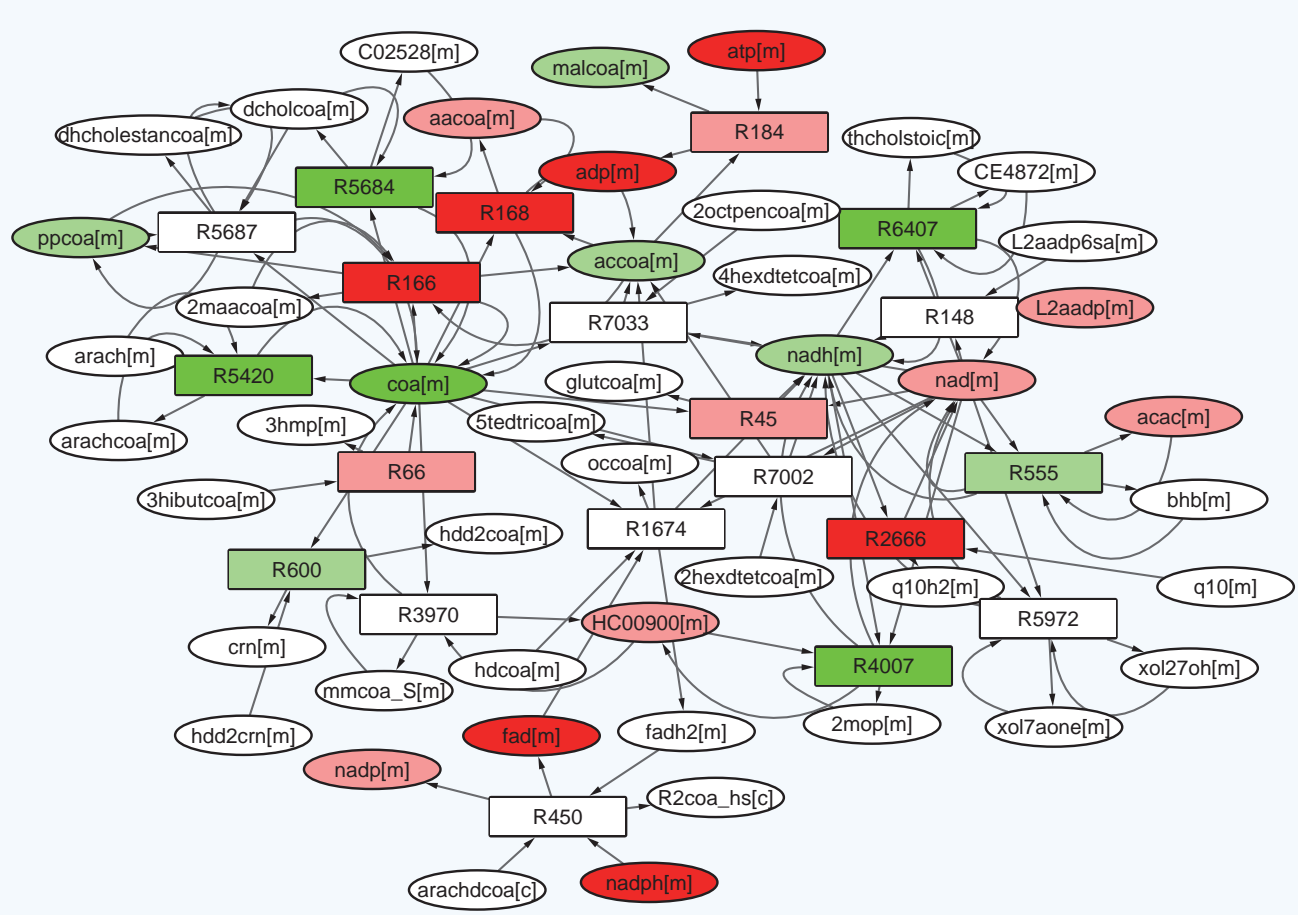

Supplementary Figure 5 Key proteins involved in lipid metabolism such as ACAT2 are upregulated. As recent literature has demonstrated significant changes in bile acid metabolism after RYGB, our findings follow suit as we observed a significant increase in key bile acid proteins such as BAAT, which is directly involved in the formation of bile acid-amino acid conjugates. An increase in bile acids throughout the gastrointestinal tract may lead to enhanced lipid and fat-soluble vitamin absorption. 


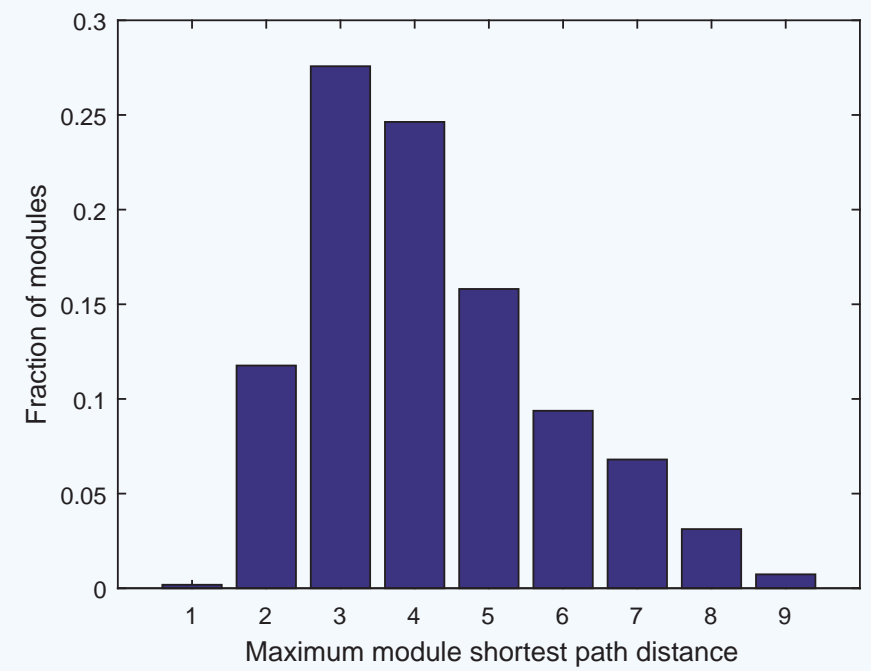

Supplementary Figure 6 The distribution of the maximum module shortest path distance between two nodes for randomly generated sub-networks of size 20 .

\section{REFERENCES}

S1. Fernández-Real, J.M., Mercader, J.M., Ortega, F.J., Moreno-Navarrete, J.M., LópezRomero, P. et al. Transferrin receptor-1 gene polymorphisms are associated with type 2 diabetes. Eur. J. Clin. Invest. 40, 600-607 (2010)

S2. Poitou, C., Perret, C., Mathieu, F., Truong, V., Blum, Y. et al. Bariatric surgery induces disruption in inammatory signaling pathways mediated by immune cells in adipose tissue: A RNA-Seq study. PLoS One 10, e0125718 (2015).

S3. Pan, X., Grigoryeva, L., Seyrantepe, V., Peng, J., Kollmann, K. et al. Serine carboxypeptidase SCPEP1 and Cathepsin a play complementary roles in regulation of vasoconstriction via inactivation of endothelin-1. PLoS Genet. 10, e1004146 (2014).

S4. Moreno-Navarrete, J.M., Blasco, G., Xifra, G., Karczewska-Kupczewska, M., Stefanowicz, M. et al. Obesity is associated with gene expression and imaging markers of iron accumulation in skeletal muscle. J. Clin. Endocrinol. Metab. 101, 1282-1289 (2016).

S5. Han, J., Jung, S., Jang, J., Kam, T.I., Choi, H. et al. Ociad2 activates $\gamma$-secretase to enhance amyloid $\beta$ production by interacting with nicastrin. Cell. Mol. Life Sci. 71, 2561-2576 (2014)

S6. Andreasen, C.H., Mogensen, M.S., Borch-Johnsen, K., Sandbaek, A., Lauritzen, T. et al. Studies of CTNNBI1 and FDFT1 variants and measures of obesity: Analyses of quantitative traits and casecontrol studies in 18,014 Danes. BMC Med. Genet. 10, 17 (2009).

S7. Challa, T.D., Straub, L.G., Balaz, M., Kiehlmann, E., Donze, O. et al. Regulation of de novo adipocyte differentiation through crosstalk between adipocytes and pre-adipocytes. Diabetes 64, 4075-4087 (2015)

S8. Nagamani, S.C., Erez, A. \& Lee, B. Argininosuccinate lyase deficiency. Genet. Med. 14, 501-507 (2012)

S9. Ward, C., Martinez-Lopez, N., Otten, E.G., Carroll, B., Maetzel, D. et al. Autophagy, lipophagy and lysosomal lipid storage disorders. Biochim. Biophys. Acta 1861, 269-284 (2016).

S10. Braverman, N., Lin, P., Moebius, F.F., Obie, C., Moser, A. et al. Mutations in the gene encoding $3 \beta$-hydroxysteroid- $\delta 8$, $\delta$-isomerase cause $X$-linked dominant ConradiHünermann syndrome. Nat. Genet. 22, 291-294 (1999).

S11. Pandey, A.K. \& Kar, S.K. REM sleep deprivation of rats induces acute phase response in liver. Biochem. Biophys. Res. Commun. 410, 242-246 (2011).

S12. Solans, A., Estivill, X. \& de La Luna, S. Cloning and characterization of human FTCD on 21q22. 3, a candidate gene for glutamate formiminotransferase deficiency. Cytogenet. Cell. Genet. 88, 43-49 (2000).

S13. Ye, H. \& Liu, W. Transcriptional networks implicated in human nonalcoholic fatty liver disease. Mol. Genet. Genomics 290, 1793-1804 (2015).

S14. Elmendorf, J.S. Fretting about fat: A new look at the lipid droplet surface and the roundabout role of Plin2 in cellular lipid storage: Focus on "direct interaction of Plin2 with lipids on the surface of lipid droplets: A live cell FRET analysis". Am. J. Physiol. Cell Physiol. 303, C713-C714 (2012).

S15. Houten, S.M., Te Brinke, H., Denis, S., Ruiter, J.P., Knegt, A.C. et al. Genetic basis of hyperlysinemia. Orphanet J. Rare Dis. 8, 57 (2013).

S16. Ohtawa, M., Yamazaki, H., Ohte, S., Matsuda, D., Ohshiro, T. et al. Synthesis and structure-activity relationship of pyripyropene $A$ derivatives as potent and selective

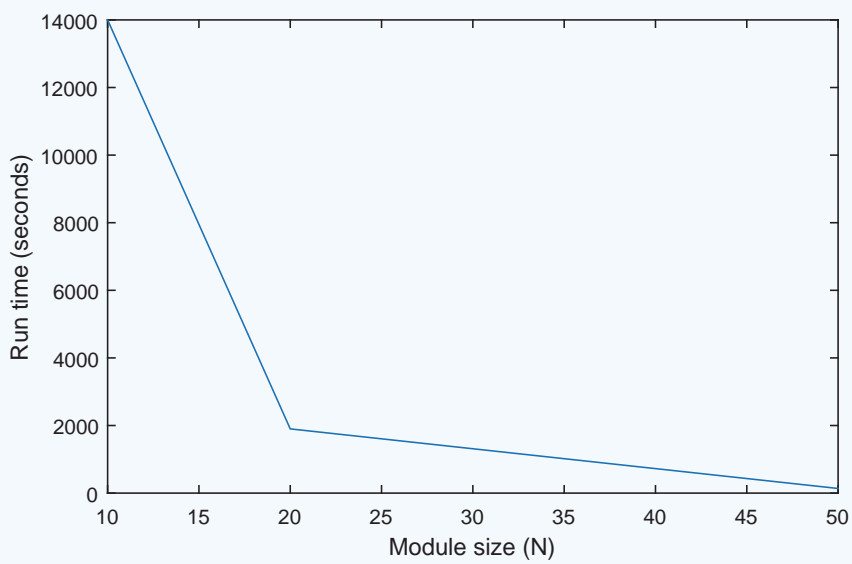

Supplementary Figure 7 Based on the module presented in Fig. 4a highlighting bile acid metabolism, we found a network-based relationship between Baat, Slc27a5, and Enpp3. We tested how much runtime would be required to identify a module encompassing all three of these enzymes at various module sizes using the seed reaction (Rs) as Baat.

acyl-CoA: cholesterol acyltransferase 2 (ACAT2) inhibitors: Part 1. Bioorg. Med. Chem Lett. 23, 1285-1287 (2013)

S17. Guillem, V., Hernández-Boluda, J.C., Gallardo, D., Buño, I., Bosch, A. et al. A polymorphism in the TYMP gene is associated with the outcome of HLA-identical sibling allogeneic stem cell transplantation. Am. J. Hematol 88, 883-889 (2013).

S18. Sahini, N., Selvaraj, S. \& Borlak, J. Whole genome transcript profiling of drug induced steatosis in rats reveals a gene signature predictive of outcome. PLoS One $\mathbf{9}, \mathrm{e} 114085$ (2014)

S19. Halpain, S. \& Dehmelt, L. The MAP1 family of microtubule-associated proteins. Genome Biol. 7, 224 (2006).

S20. Recalcati, S., Tacchini, L., Alberghini, A., Conte, D. \& Cairo, G. Oxidative stressmediated down-regulation of rat hydroxyacid oxidase 1, a liver-specific peroxisomal enzyme. Hepatology 38, 1159-1166 (2003)

S21. Zhang, J., Song, M., Wang, J., Sun, M., Wang, B. et al. Enoyl coenzyme a hydratase is an important factor in the lymphatic metastasis of tumors. Biomed. Pharmacother 65, 157-162 (2011).

S22. Son, J., Lyssiotis, C.A., Ying, H., Wang, X., Hua, S. et al. Glutamine supports pancreatic cancer growth through a KRAS-regulated metabolic pathway. Nature 496, 101-105 (2013).

S23. Wells, M.A., Vendrov, K.C., Edin, M.L., Ferslew, B.C., Zha, W. et al. Characterization of the cytochrome $\mathrm{p} 450$ epoxyeicosanoid pathway in non-alcoholic steatohepatitis. Prostaglandins Other Lipid Mediat. 125, 19-29 (2016).

S24. Schiöth, H.B., Boström, A., Murphy, S.K., Erhart, W., Hampe, J. et al. A targeted analysis reveals relevant shifts in the methylation and transcription of genes responsible for bile acid homeostasis and drug metabolism in non-alcoholic fatty liver disease. BMC Genom. 17, 462 (2016)

S25. Zhuo, S., Yang, M., Zhao, Y., Chen, X., Zhang, F. et al. MicroRNA-451 negatively regulates hepatic glucose production and glucose homeostasis by targeting glycerol kinase mediated gluconeogenesis. Diabetes 65, 3276-3288 (2016).

S26. Crujeiras, A., Parra, D., Goyenechea, E. \& Martínez, J. Sirtuin gene expression in human mononuclear cells is modulated by caloric restriction. Eur. J. Clin. Invest. 38, 672-678 (2008)

S27. Ganu, R.S., Ishida, Y., Koutmos, M., Kolokotronis, S.O., Roca, A.L. et al. Evolutionary analyses and natural selection of betaine-homocysteine S-methyltransferase (BHMT) and BHMT2 genes. PLoS One 10, e0134084 (2015).

S28. Wu, J., Wang, C., Li, S., Li, S., Wang, W. et al. Thyroid hormone-responsive SPOT 14 homolog promotes hepatic lipogenesis, and its expression is regulated by liver $\mathrm{X}$ receptor $\alpha$ through a sterol regulatory element-binding protein $1 c$-dependent mechanism in mice. Hepatology 58, 617-628 (2013).

S29. Cha, Y.J., Kim, D.H., Jung, W.H. \& Koo, J.S. Expression of sarcosine metabolismrelated proteins according to metastatic site in breast cancer. Int. J. Clin. Exp. Pathol. 7, 7824-7833 (2014).

S30. Lin, M. \& Napoli, J.L. cDNA cloning and expression of a human aldehyde dehydrogenase $(A L D H)$ active with 9-cis-retinal and identification of a rat ortholog, ALDH12. J. Biol. Chem. 275, 40106-40112 (2000). 
S31. Olsvik, P., Kristensen, T., Waagbø, R., Rosseland, B., Tollefsen, K.E. et al. mRNA expression of antioxidant enzymes (SOD, CAT and GSH-Px) and lipid peroxidative stress in liver of Atlantic salmon (Salmo salar) exposed to hyperoxic water during smoltification. Comp. Biochem. Physiol. Part C Toxicol. Pharmacol. 141, 314-323 (2005).

S32. Pushkin, A., Carpenito, G., Abuladze, N., Newman, D., Tsuprun, V. et al. Structural characterization, tissue distribution, and functional expression of murine aminoacylase III. Am. J. Physiol. Cell Physiol. 286, C848-C856 (2004)

S33. Wang, C., Tao, Q., Wang, X., Wang, X. \& Zhang, X. Impact of high-fat diet on liver genes expression profiles in mice model of nonalcoholic fatty liver disease. Environ. Toxicol. Pharmacol. 45, 52-62 (2016).

S34. Meng, X., Tackmann, N.R., Liu, S., Yang, J., Dong, J. et al. RPL23 links oncogenic RAS signalling to p53-mediated tumor suppression. Cancer Res. 76, 5030-5039 (2016)

S35. Selvaraju, V., Markandaya, M., Prasad, P.V.S., Sathyan, P., Sethuraman, G. et al. Mutation analysis of the cathepsin $\mathrm{c}$ gene in Indian families with Papillon-Lefèvre syndrome. BMC Med. Genet. 4, 5 (2003).

S36. Lee, S.J., Kim, S.H., Park, K.M., Lee, J.H. \& Park, J.W. Increased obesity resistance and insulin sensitivity in mice lacking the isocitrate dehydrogenase 2 gene. Free Radic. Biol. Med. 99, 179-188 (2016).

S37. Korekane, H., Park, J.Y., Matsumoto, A., Nakajima, K., Takamatsu, S. et al. Identification of ectonucleotide pyrophosphatase/phosphodiesterase 3 (ENPP3) as a regulator of $\mathrm{N}$-acetylglucosaminyltransferase GnT-IX (GnT-Vb). J. Biol. Chem. 288, 27912-27926 (2013).

S38. Duscher, S., Peters, U. \& Brenig, B. A polymorphic microsatellite located within the second intron of the methylmalonyl-CoA mutase (MUT) gene on SSC 1. Anim. Genet. 31, 339 (2000).

S39. Hiles, R.A. \& Henderson, L.M. The partial purification and properties of hydroxylysine kinase from rat liver. J. Biol. Chem. 247, 646-651 (1972).

S40. Ghanbari, M., Franco, O.H., de Looper, H., Hofman, A., Erkeland, S. et al. Genetic variations in microRNA binding sites affect microRNA-mediated regulation of several genes associated with cardiometabolic phenotypes. Circ. Cardiovasc. Genet. 8, 473-486 (2015)

S41. Park, J.E., Kim, H.T., Lee, S., Lee, Y.S., Choi, U.K. et al. Differential expression of intermediate filaments in the process of developing hepatic steatosis. Proteomics 11, 2777-2789 (2011).

S42. Wiedl, T., Arni, S., Roschitzki, B., Grossmann, J., Collaud, S. et al. Activity-based proteomics: Identification of ABHD11 and ESD activities as potential biomarkers for human lung adenocarcinoma. J. Proteomics 74, 1884-1894 (2011).

S43. Liu, Y.F., Yang, A., Liu, W., Wang, C., Wang, M. et al. NME2 reduces proliferation, migration and invasion of gastric cancer cells to limit metastasis. PLoS One 10, e0115968 (2015)

S44. Bjursell, M.K., Blom, H.J., Cayuela, J.A., Engvall, M.L., Lesko, N. et al. Adenosine kinase deficiency disrupts the methionine cycle and causes hypermethioninemia, encephalopathy, and abnormal liver function. Am. J. Human Genet. 89, 507-515 (2011).

S45. Zhu, R., Baker, S.S., Moylan, C.A., Abdelmalek, M.F., Guy, C.D. et al. Systematic transcriptome analysis reveals elevated expression of alcohol-metabolizing genes in NAFLD livers. J. Pathol. 238, 531-542 (2016).

S46. Bondia-Pons, I., Boqué, N., Paternain, L., Santamaría, E., Fernández, J. et al. Liver proteome changes induced by a short-term high-fat sucrose diet in wistar rats. J. Nutrigenet. Nutrigenom. 4, 344-353 (2012).

S47. Hanioka, N., Gonzalez, F., Lindberg, N., Liu, G., Gelboin, H. et al. Site-directed mutagenesis of cytochrome p450s CYP2A1 and CYP2A2: Influence of the distal helix on the kinetics of testosterone hydroxylation. Biochemistry 31, 3364-3370 (1992).

S48. Katashima, R., Iwahana, H., Fujimura, M., Yamaoka, T. \& Itakura, M. Assignment of the human phosphoribosylpyrophosphate synthetase-associated protein 41 gene (PRPSAP2) to 17p11.2-p12. Genomics 54, 180-181 (1998).

S49. Scarlet, D., Ertl, R., Aurich, C. \& Steinborn, R. The orthology clause in the next generation sequencing era: Novel reference genes identified by RNA-seq in humans improve normalization of neonatal equine ovary RT-qPCR data. PLoS One 10, e0142122 (2015)

S50. Chen, T., Wu, Q., Zhang, Y. \& Zhang, D. NDUFV2 regulates neuronal migration in the developing cerebral cortex through modulation of the multipolar-bipolar transition. Brain Res. 1625, 102-110 (2015).

S51. Liao, C.C., Lin, Y.L. \& Kuo, C.F. Effect of high-fat diet on hepatic proteomics of hamsters J. Agric. Food Chem. 63, 1869-1881 (2015).

S52. Koukouritaki, S.B., Simpson, P., Yeung, C.K., Rettie, A.E. \& Hines, R.N. Human hepatic flavin-containing monooxygenases 1 (FMO1) and 3 (FMO3) developmental expression. Pediatr. Res. 51, 236-243 (2002).

S53. Goh, D.L., Patel, A., Thomas, G.H., Salomons, G.S., Schor, D.S. et al. Characterization of the human gene encoding alpha-aminoadipate aminotransferase (AADAT). Mol. Genet. Metab. 76, 172-180 (2002)

S54. Honda, K. The biological role of actinin-4 (ACTN4) in malignant phenotypes of cancer. Cell Biosci. 5, 41 (2015).

S55. Lake, A.D., Novak, P., Shipkova, P., Aranibar, N., Robertson, D. et al. Decreased hepatotoxic bile acid composition and altered synthesis in progressive human nonalcoholic fatty liver disease. Toxicol. Appl. Pharmacol. 268, 132-140 (2013).
S56. Agafonov, D.E., Kolb, V.A. \& Spirin, A.S. Proteins on ribosome surface: Measurements of protein exposure by hot tritium bombardment technique. Proc. Natl. Acad. Sci. U.S.A. 94, 12892-12897 (1997).

S57. Chou, J.Y. \& Mansfield, B.C. The SLC37 family of sugar-phosphate/phosphate exchangers. Curr. Top. Membr. 73, 357-382 (2014).

S58. Taketani, S., Furukawa, T. \& Furuyama, K. Expression of coproporphyrinogen oxidase and synthesis of hemoglobin in human erythroleukemia k562 cells. Eur. J. Biochem. 268, 1705-1711 (2001).

S59. Fisher, C.D., Lickteig, A.J., Augustine, L.M., Ranger-Moore, J., Jackson, J.P. et al. Hepatic cytochrome p450 enzyme alterations in humans with progressive stages of nonalcoholic fatty liver disease. Drug Metab. Dispos. 37, 2087-2094 (2009).

S60. Phatarphekar, A. \& Rokita, S.E. Functional analysis of iodotyrosine deiodinase from drosophila melanogaster. Protein Sci. 25, 2187-2195 (2016).

S61. Kang, H.W., Niepel, M.W., Han, S., Kawano, Y. \& Cohen, D.E. Thioesterase superfamily member 2/acyl-CoA thioesterase 13 (Them2/Acot13) regulates hepatic lipid and glucose metabolism. FASEB J. 26, 2209-2221 (2012).

S62. Han, J., Zhang, M., Froese, S., Dai, F.F., Robitaille, M. et al. The identification of novel protein-protein interactions in liver that affect glucagon receptor activity. PLoS One 10, e0129226 (2015)

S63. Kohjima, M., Enjoji, M., Higuchi, N., Kato, M., Kotoh, K. et al. Re-evaluation of fatty acid metabolism-related gene expression in nonalcoholic fatty liver disease. Int. J. Mol. Med. 20, 351-358 (2007).

S64. Doi, R., Oishi, K. \& Ishida, N. Clock regulates circadian rhythms of hepatic glycogen synthesis through transcriptional activation of Gys2. J. Biol. Chem. 285, 22114-22121 (2010).

S65. Roberts, M.D., Mobley, C.B., Toedebush, R.G., Heese, A.J., Zhu, C. et al. Western diet-induced hepatic steatosis and alterations in the liver transcriptome in adult Brown-Norway rats. BMC Gastroenterol. 15, 151 (2015).

S66. Didona, B., Codispoti, A., Bertini, E., Rizzo, W.B., Carney, G. et al. Novel and recurrent ALDH3A2 mutations in Italian patients with Sjögren-Larsson syndrome. J. Human Genet. 52, 865-870 (2007).

S67. Duarte, M.K.R.N., de Araújo, J.N.G., Duarte, V.H.R., de Oliveira, K.M., de Oliveira, J.M et al. The relationship of the oleic acid level and ECHDC3 mRNA expression with the extent of coronary lesion. Lipids Health Dis. 15, 144 (2016).

S68. Pashaj, A., Yi, X., Xia, M., Canny, S., Riethoven, J.J.M. et al. Characterization of genomewide transcriptional changes in liver and adipose tissues of ZDF ( $\mathrm{Fa} / \mathrm{Fa}$ ) rats fed $r-\alpha$-lipoic acid by next-generation sequencing. Physiol. Genom. 45, 1136-1143 (2013).

S69. Hunt, M.C. Siponen, M.I. \& Alexson, S.E. The emerging role of acyl-CoA thioesterases and acyltransferases in regulating peroxisomal lipid metabolism. Biochim. Biophys. Acta 1822, 1397-1410 (2012)

S70. Shahbaaz, M., Ahmad, F. \& Hassan, M.I. Structure-based function analysis of putative conserved proteins with isomerase activity from Haemophilus influenzae. 3 Biotech $\mathbf{5}$ 741-763 (2015).

S71. Seale, L.A., Gilman, C.L., Hashimoto, A.C., Ogawa-Wong, A.N. \& Berry, M.J. Dietinduced obesity in the selenocysteine lyase knockout mouse. Antioxid. Redox Signal. 23, 761-774 (2015).

S72. Teye, K., Quaye, I., Koda, Y., Soejima, M., Tsuneoka, M. et al. A-61C and C-101G HP gene promoter polymorphisms are, respectively, associated with ahaptoglobinaemia and hypohaptoglobinaemia in Ghana. Clin. Genet. 64, 439-443 (2003).

S73. Flores, I.L., Kawahara, R., Miguel, M.C., Granato, D.C., Domingues, R.R. et al. EEF1D modulates proliferation and epithelial-mesenchymal transition in oral squamous cell carcinoma. Clin. Sci. 130, 785-799 (2016).

S74. Song, M.K., Lee, S.W., Suh, Y.S., Lee, K.J. \& Sung, Y.C. Enhancement of immunoglobulin G2a and cytotoxic T-lymphocyte responses by a booster immunization with recombinant hepatitis C virus E2 protein in E2 DNA-primed mice. J. Virol. 74, 2920-2925 (2000).

S75. Pogribny, I.P., Kutanzi, K., Melnyk, S., De Conti, A., Tryndyak, V. et al. Strain-dependent dysregulation of one-carbon metabolism in male mice is associated with choline- and folate-deficient diet-induced liver injury. FASEB J. 27, 2233-2243 (2013).

S76. Pihlajamaki, J., Boes, T., Kim, E.Y., Dearie, F., Kim, B.W. et al. Thyroid hormone-related regulation of gene expression in human fatty liver. J. Clin. Endocrinol. Metab. 94 3521-3529 (2009).

S77. Ishizuka, T., Fujimori, I., Kato, M., Noji-Sakikawa, C., Saito, M. et al. Human carboxymethylenebutenolidase as a bioactivating hydrolase of olmesartan medoxomil in liver and intestine. J. Biol. Chem. 285, 11892-11902 (2010).

S78. Xia, N., Tang, Z., Wang, C., Xu, G., Nie, X. et al. PCBP2 regulates hepatic insulin sensitivity via HIF-1 $\alpha$ and STAT3 pathway in HEPG2 cells. Biochem. Biophys. Res. Commun. 463, 116-122 (2015).

S79. Ikegawa, S., Isomura, M., Koshizuka, Y. \& Nakamura, Y. Cloning and characterization of human and mouse PROSC (proline synthetase co-transcribed) genes. J. Human Genet. 44, 337-342 (1999)

S80. Brolin, R., Gorman, J., Gorman, R., Petschenik, A., Bradley, L. et al. Are vitamin B12 and folate deficiency clinically important after Roux-en-Y gastric bypass? J. Gastrointest. Surg. 2, 436-442 (1998) 